\title{
Cough Sound Discrimination in Noisy and Reverberant Environments using Microphone Arrays
}

\author{
by
}

Payam Moradshahi, B.Eng., B.Sc.

A thesis submitted to the Faculty of Graduate and Postdoctoral Affairs

in partial fulfillment of the requirements for the degree of

Master of Applied Science in Biomedical Engineering

(Ottawa -Carleton Institute for Biomedical Engineering (OCIBME))

Department of Systems and Computer Engineering

Carleton University

Ottawa, Ontario, Canada, K1S 5B6

January 2012

(C) Copyright 2012, Payam Moradshahi 
Library and Archives

Canada

Published Heritage

Branch

395 Wellington Street

Ottawa ON K1A ON4

Canada
Bibliothèque et

Archives Canada

Direction du

Patrimoine de l'édition

395 , rue Wellington

Ottawa ON K1A ON4

Canada
Your file Votre référence

ISBN: 978-0-494-87802-6

Our file Notre référence

ISBN: 978-0-494-87802-6

\section{NOTICE:}

The author has granted a nonexclusive license allowing Library and Archives Canada to reproduce, publish, archive, preserve, conserve, communicate to the public by telecommunication or on the Internet, loan, distrbute and sell theses worldwide, for commercial or noncommercial purposes, in microform, paper, electronic and/or any other formats.

The author retains copyright ownership and moral rights in this thesis. Neither the thesis nor substantial extracts from it may be printed or otherwise reproduced without the author's permission.
AVIS:

L'auteur a accordé une licence non exclusive permettant à la Bibliothèque et Archives Canada de reproduire, publier, archiver, sauvegarder, conserver, transmettre au public par télécommunication ou par l'Internet, prêter, distribuer et vendre des thèses partout dans le monde, à des fins commerciales ou autres, sur support microforme, papier, électronique et/ou autres formats.

L'auteur conserve la propriété du droit d'auteur et des droits moraux qui protege cette thèse. $\mathrm{Ni}$ la thèse ni des extraits substantiels de celle-ci ne doivent être imprimés ou autrement reproduits sans son autorisation.
In compliance with the Canadian Privacy Act some supporting forms may have been removed from this thesis.

While these forms may be included in the document page count, their removal does not represent any loss of content from the thesis.
Conformément à la loi canadienne sur la protection de la vie privée, quelques formulaires secondaires ont été enlevés de cette thèse.

Bien que ces formulaires aient inclus dans la pagination, il n'y aura aucun contenu manquant. 


\section{Abstract}

Cough sound discriminator algorithms are capable of distinguishing between dry and wet cough types. The performance of such algorithms, however, is affected by noise and reverberation which might exist in patients' environments. In this thesis, the performance of the previously developed cough sound discriminator in a noisy and reverberant room is quantitatively measured using Linear Separation Score. Experiments revealed a significant decrease in the performance of the cough sound discriminator in the presence of noise and reverberation using a single microphone for the cough sound acquisition. In order to improve this performance, a microphone array structure which included a maximum of 7 microphones was designed with a delay-and-sum beamformer. Experiments showed a significant improvement in the performance of the cough sound discriminator using a microphone array in noisy and reverberant environments. Finally, a Graphical User Interface was developed in order to visualize the beampattern emitted by the microphone array structure. 


\section{Acknowledgments}

I would like to thank my supervisor, Dr. Rafik Goubran, for his guidance, encouragement and offering me his technical expertise throughout my thesis. I would also like to acknowledge the financial support from Natural Sciences and Engineering Research Council (NSERC), Healthcare Support through Information Technology Enhancements (hSITE) and Carleton University.

I would like to thank Dr. Vilas Joshi for his friendship and sharing his knowledge.

I would like to thank my parents Houshang and Shokat for their love and support throughout my life and teaching me the value of education from early age.

I would like to thank my brother, Peyman, for being my role model throughout my life. Trying to follow in his footsteps has always been a source of motivation for me.

Finally, I would like to thank my wife, Hanieh Chatrzarrin. Her love and support and offering her technical knowledge during my undergraduate and graduate years has helped me achieve my academic goals. Thank you for being the best classmate, colleague, friend and life partner I could have ever asked for. 


\section{Table of Contents}

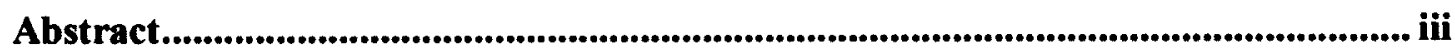

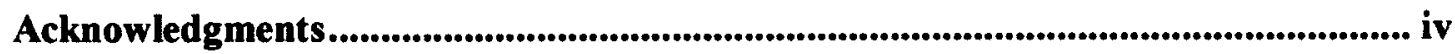

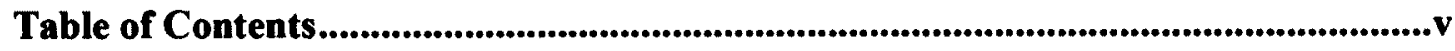

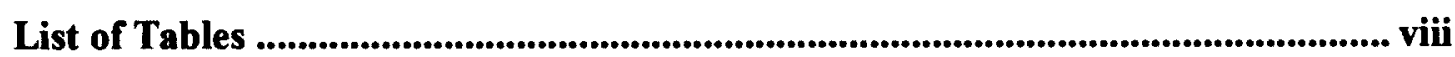

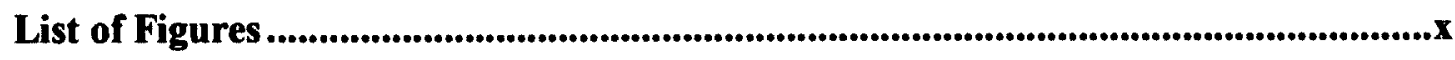

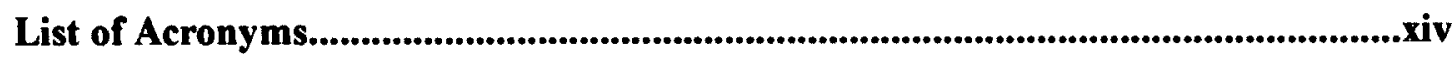

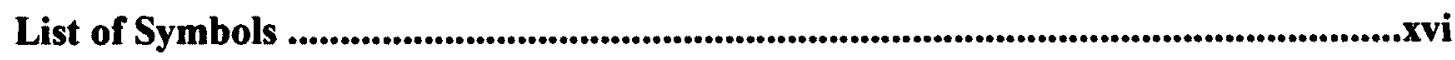

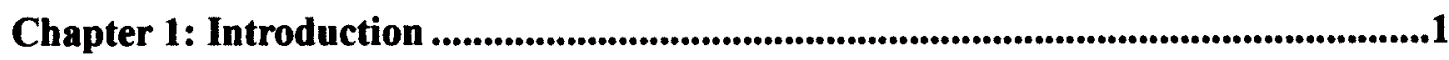

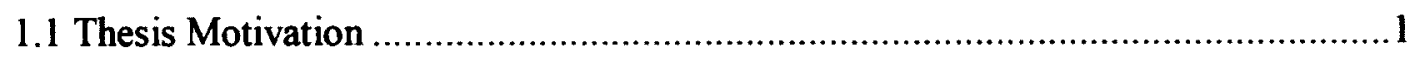

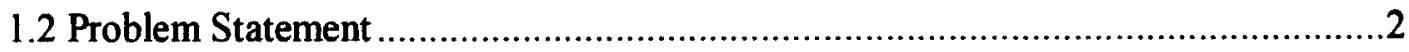

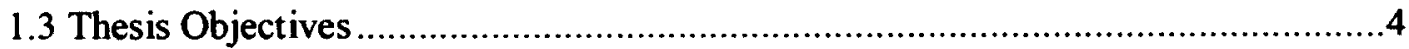

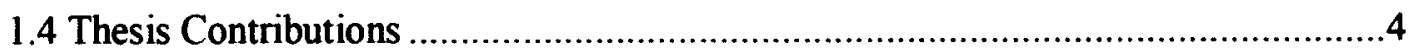

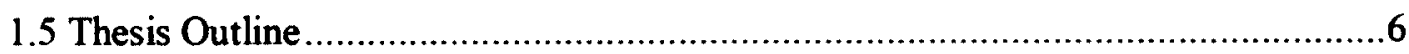

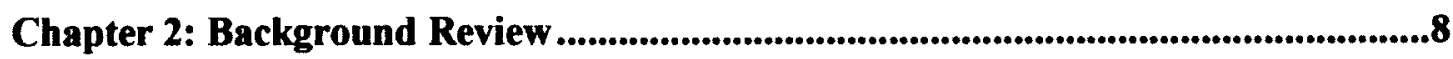

2.1 Microphone Arrays and Beamforming Techniques........................................9

2.2 Sound Acquisition using Microphone Arrays ............................................. 12

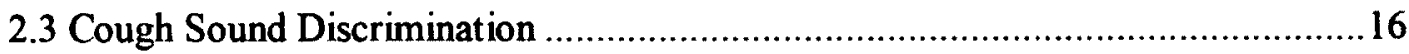




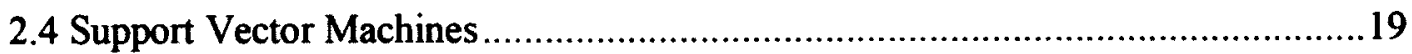

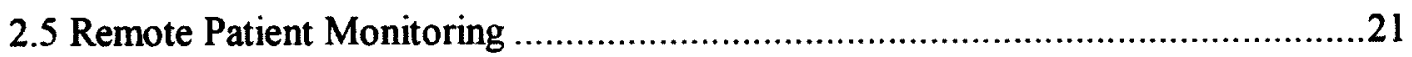

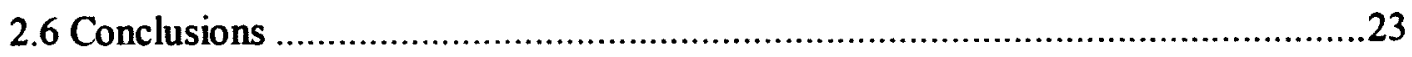

Chapter 3: Cough Sound Discriminator Performance .....................................................25

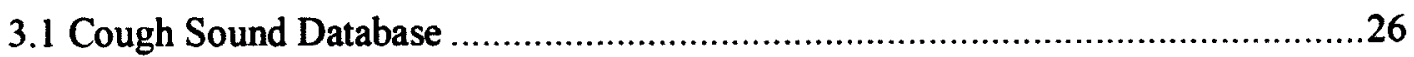

3.2 Linear Separability of Cough Sound Discriminator .............................................26

3.3 Linear Separation using Support Vector Machines ..............................................28

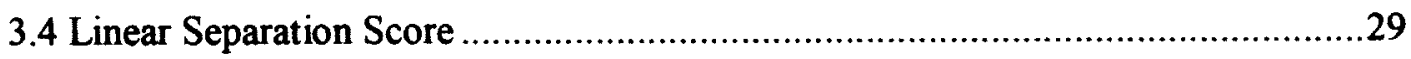

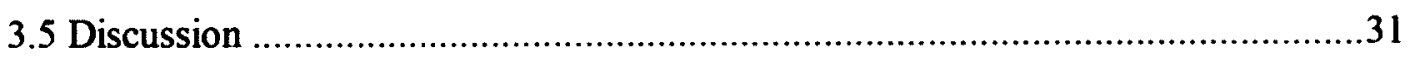

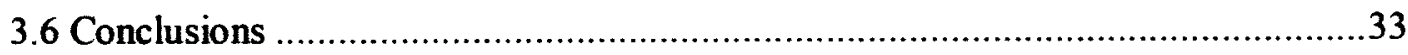

Chapter 4: Cough Sound Acquisition using a Single Microphone .................................34

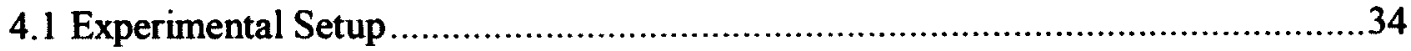

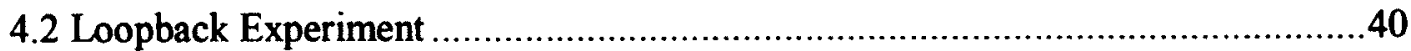

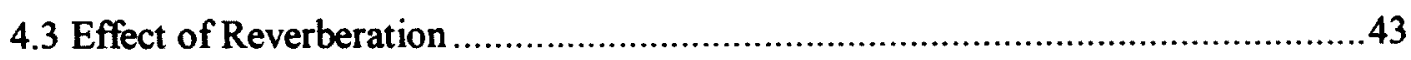

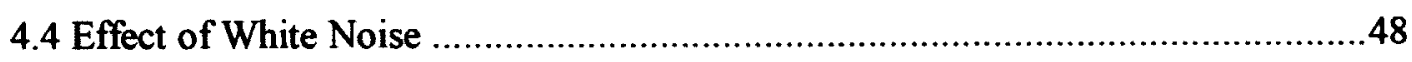

4.4.1 Microphone to Speaker Distance ..............................................................49

4.4.2 Cough Sound Volume Level ...................................................................51

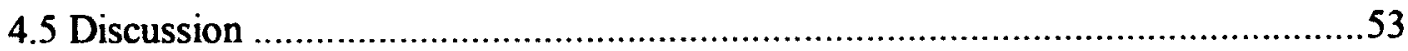

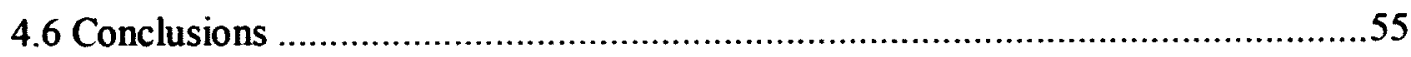

Chapter 5: Cough Sound Acquisition using Microphone Array ....................................58

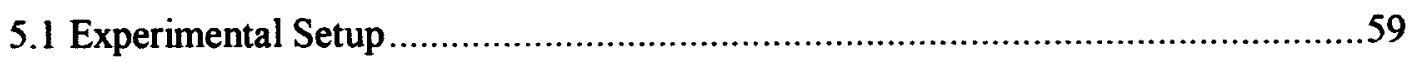

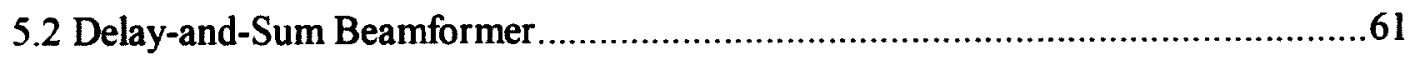

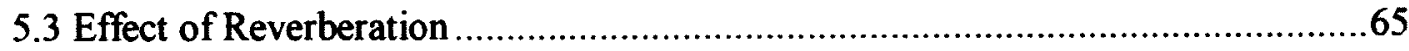

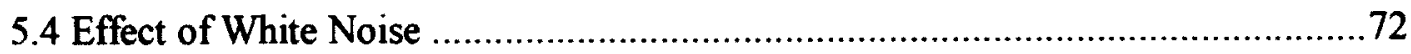

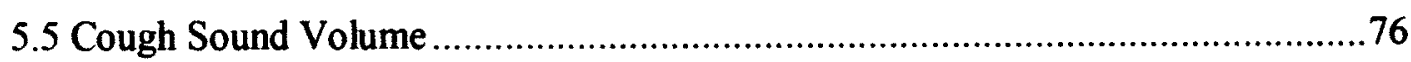


5.6 Discussions

5.7 Conclusions

Chapter 6: Microphone Array and Beamforming Graphical User Interface ............85

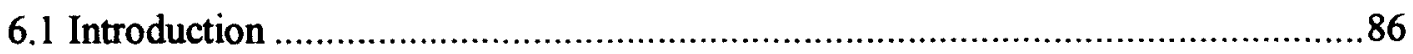

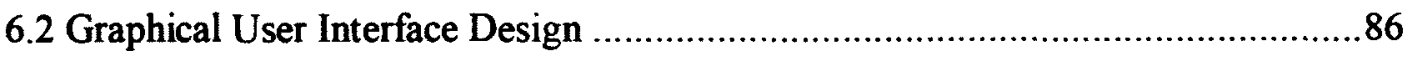

6.3 Beampattern and Delay-and-Sum Beamformer ............................................... 88

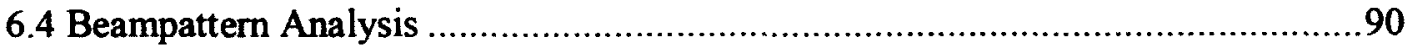

6.4.1 Microphone-to-Speaker Distance …………………………………….....91

6.4.2 Microphone-to-Microphone Distance ……………....................................

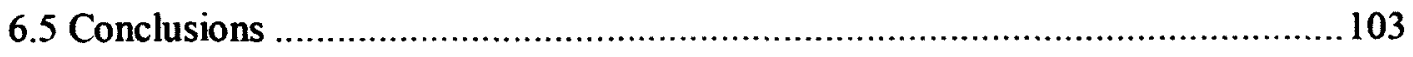

Chapter 7: Conclusion ..............................................................................................................104

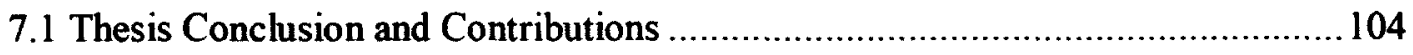

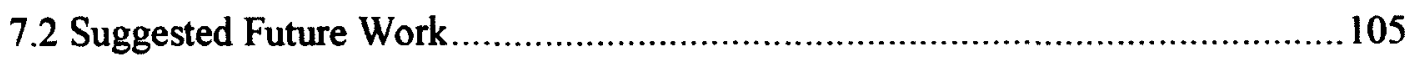

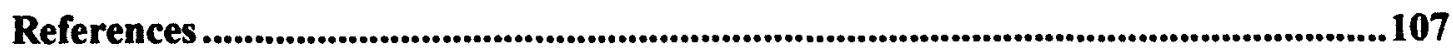




\section{List of Tables}

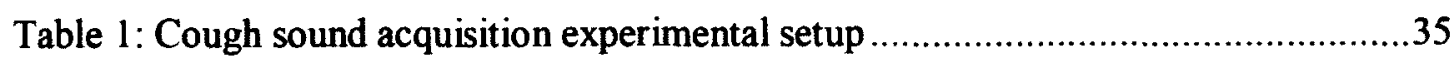

Table 2: Single microphone cough sound acquisition Linear Separation Score ..............47

Table 3: LSS of single microphone cough sound acquisition with and without white noise

Table 4: LSS of single microphone cough sound acquisition with white noise and

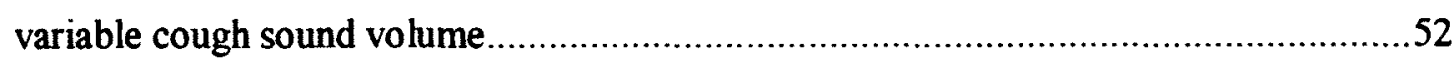

Table 5: Microphone array cough sound acquisition experimental variables .................60

Table 6: LSS for microphone array cough sound acquisition $\left(D_{\text {Micto-Speaker }}=150 \mathrm{~cm}\right) \ldots . .66$

Table 7: LSS for microphone array cough sound acquisition $\left(D_{\text {Mic-to-Speaker }}=200 \mathrm{~cm}\right) \ldots . .67$

Table 8: LSS for microphone array cough sound acquisition $\left(\mathrm{D}_{\text {Mic-to-Speaker }}=250 \mathrm{~cm}\right) \ldots . .68$

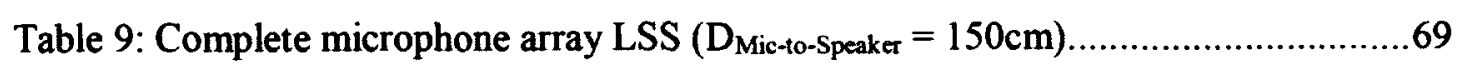

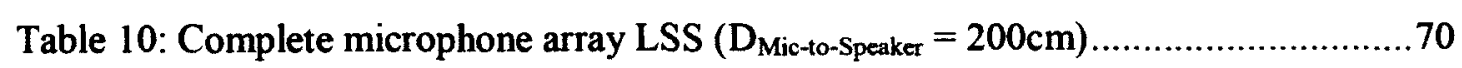

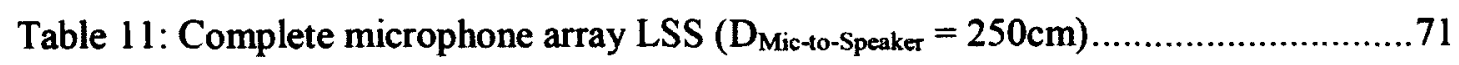

Table 12: Microphone array LSS with white noise $\left(D_{\text {Mic-to-Speaker }}=50 \mathrm{~cm}\right) \ldots \ldots \ldots \ldots \ldots \ldots . . \ldots \ldots$

Table 13: Microphone array LSS with white Noise $\left(D_{\text {Mic-to-Speaker }}=200 \mathrm{~cm}\right) \ldots \ldots \ldots \ldots \ldots . . . . .75$ 
Table 14: LSS of microphone array cough sound acquisition with white noise and

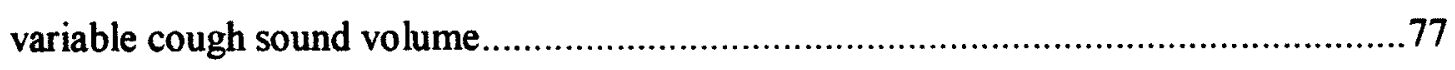




\section{List of Figures}

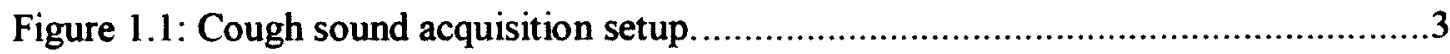

Figure 2.1: Linear microphone array arrangement ..................................................10

Figure 2.2: Feature 1 of cough sound discriminator (Taken with permission from [16]). 18

Figure 2.3: Feature 2 of cough sound discriminator (Taken with permission from [16]). 18

Figure 2.4: Cough sound discriminator result (Taken with permission from [16]) .........19

Figure 2.5: Support Vector Machine ..........................................................................21

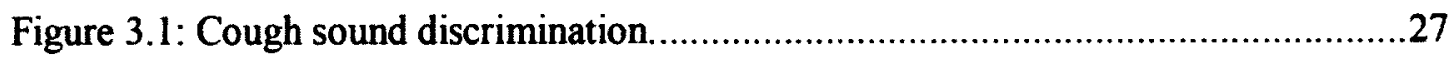

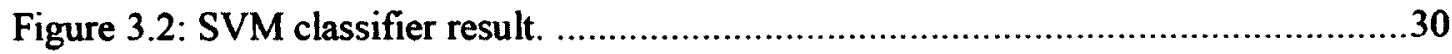

Figure 3.3: Linear Separation Score system. ..........................................................31

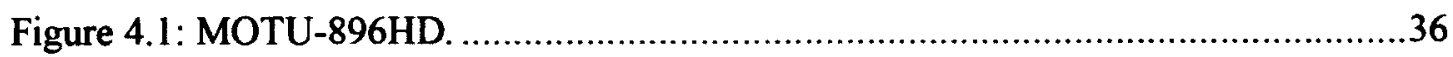

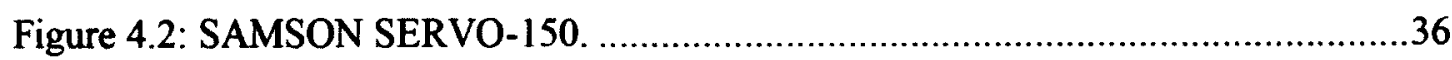

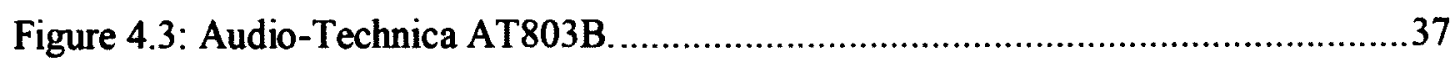

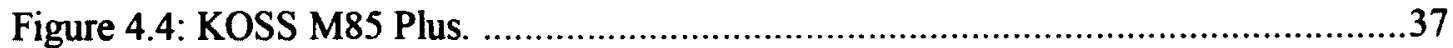

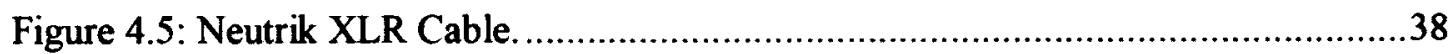

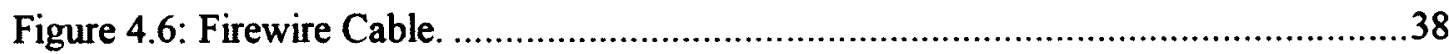




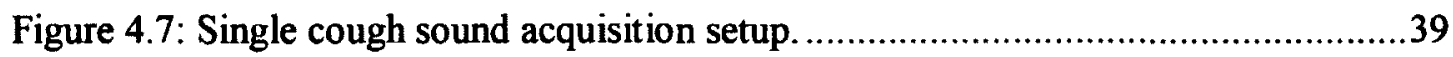

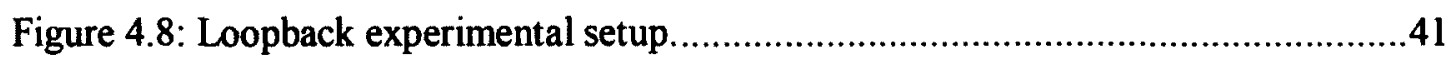

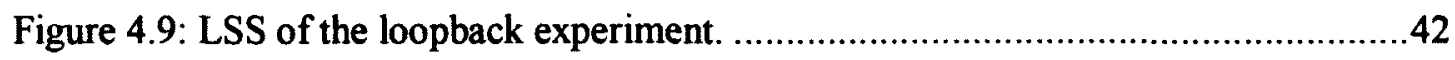

Figure 4.10: Single microphone cough sound acquisition $\left(D_{\text {mic-to-speaker }}=50 \mathrm{~cm}\right) \ldots \ldots \ldots . . .45$

Figure 4.11: Single microphone cough sound acquisition $\left(D_{\text {mic-to-speaker }}=100 \mathrm{~cm}\right) \ldots \ldots . .45$

Figure 4.12: Single microphone cough sound acquisition $\left(D_{\text {mic-to-speaker }}=150 \mathrm{~cm}\right) \ldots \ldots \ldots 46$

Figure 4.13: Single microphone cough sound acquisition $\left(D_{\text {mic-to-speaker }}=200 \mathrm{~cm}\right) \ldots \ldots . .46$

Figure 4.14: Single microphone cough sound acquisition $\left(D_{\text {mic-to-speaker }}=250 \mathrm{~cm}\right) \ldots \ldots \ldots . . .47$

Figure 4.15: Single microphone cough sound acquisition with added white noise. ........49

Figure 4.16: LSS of single-microphone and white noise $\left(\mathrm{D}_{\text {mic-to-speaker }}=50 \mathrm{~cm}\right) \ldots \ldots \ldots \ldots . \ldots 50$

Figure 4.17: LSS of single-microphone and white noise $\left(D_{\text {mic-to-speaker }}=200 \mathrm{~cm}\right) \ldots \ldots \ldots . .51$

Figure 4.18: LSS of variable cough volume with single microphone.......................52

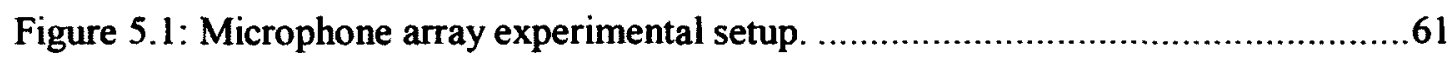

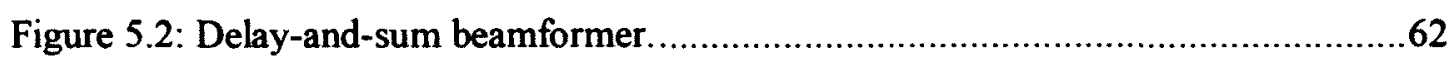

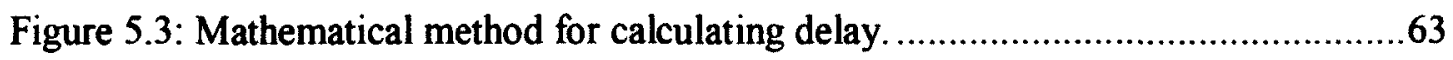

Figure 5.4: Experimental method for calculating delay. ........................................64

Figure 5.5: Microphone array cough sound acquisition $\left(D_{\text {Mic-2-Speaker }}=150 \mathrm{~cm}\right) \ldots \ldots \ldots \ldots 6$

Figure 5.6: Microphone array cough sound acquisition $\left(D_{\text {Mie-2-Speaker }}=200 \mathrm{~cm}\right) \ldots \ldots \ldots \ldots . .67$

Figure 5.7: Microphone array cough sound acquisition $\left(\mathrm{D}_{\text {Mic-2-Speaker }}=250 \mathrm{~cm}\right) \ldots \ldots \ldots \ldots . \ldots 8$

Figure 5.8: Microphone array plus white noise experimental setup. ...........................73 
Figure 5.9: Microphone array cough sound acquisition and white noise $\left(D_{\text {Mic-2-Speaker }}=\right.$ $50 \mathrm{~cm})$ 74

Figure 5.10: Microphone array cough sound acquisition and white noise $\left(\mathrm{D}_{\text {Mic-2-Speaker }}=\right.$ $200 \mathrm{~cm})$ .75

Figure 5.11: Microphone array cough sound acquisition with white noise and variable cough sound volume. 77

Figure 6.1: Graphical User Interface. 87

Figure 6.2: Manual microphone placement pane 89

Figure 6.3: Sample beampattern diagram (linear mode). .90

Figure 6.4: Beampattern for $D_{\text {Mic-to-Speaker }}=10 \mathrm{~cm}$ .93

Figure 6.5: Beampattern for $D_{\text {Mic-to-Speaker }}=30 \mathrm{~cm}$. 94

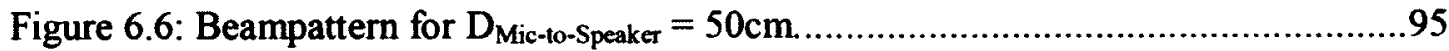

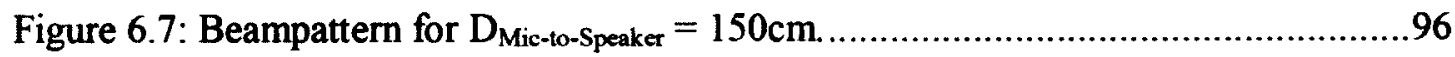

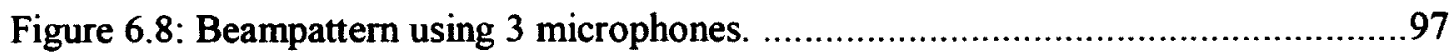

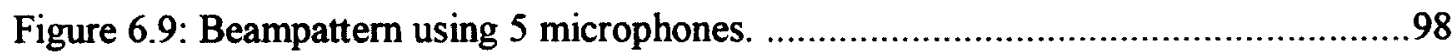

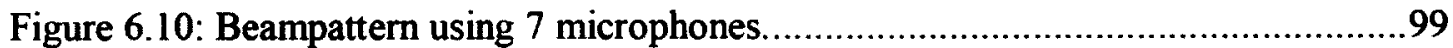

Figure 6.11: Beampattern at $2 \mathrm{kHz}$ with 3 microphones and microphone-to-microphone distance of a) $5 \mathrm{~cm} \mathrm{b)} 10 \mathrm{~cm}$, c) $15 \mathrm{~cm}$ and d) $20 \mathrm{~cm}$. 100 Figure 6.12: Beampattern at $2 \mathrm{kHz}$ with 5 microphones and microphone-to-microphone distance of a) $5 \mathrm{~cm} \mathrm{b)} 10 \mathrm{~cm}$, c) $15 \mathrm{~cm}$ and d) $20 \mathrm{~cm}$. 101 
Figure 6.13: Beampattern at $2 \mathrm{kHz}$ with 7 microphones and microphone-to-microphone

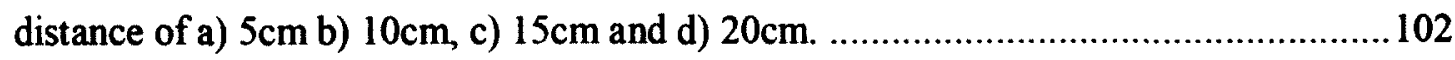




\section{List of Acronyms}

\begin{tabular}{|c|c|}
\hline AED & Audio Event Detection \\
\hline ANN & Artificial Neural Network \\
\hline APR & Adaptive Pitch Ratio \\
\hline DAS & Delay-and-Sum \\
\hline DOA & Direction of Arrival \\
\hline DSP & Digital Signal Processing \\
\hline FFT & Fast Fourier Transform \\
\hline GUI & Graphical User Interface \\
\hline ICA & Independent Component Analysis \\
\hline LPC & Linear Predictive Coefficient \\
\hline LSS & Linear Separation Score \\
\hline MA & Microphone Array \\
\hline PDA & Personal Digital Assistance \\
\hline RPM & Remote Patient Monitoring \\
\hline SNR & Signal-to-Noise Ratio \\
\hline
\end{tabular}




\section{SVM Support Vector Machine}




\section{List of Symbols}

$\begin{array}{ll}\text { b } & \text { y-intercept of hyperplane } \\ \text { D } & \text { Distance between microphones } \\ \text { dB } & \text { Decibel } \\ \text { Fs } & \text { Sampling frequency } \\ \text { i } & \text { Index } \\ \text { N } & \text { Number of microphone } \\ \pi & \text { Hyperplane } \\ \text { S }(t) & \text { Signal in time domain } \\ \theta & \text { Direction of arrival } \\ v & \text { Speed of sound } \\ \text { w } & \text { Slope of hyperplane } \\ \text { X } & \text { Distance between speaker and microphone }\end{array}$

xvi 


\section{Chapter 1:}

\section{Introduction}

\subsection{Thesis Motivation}

The use of microphone arrays has gained popularity in systems which require high quality sound acquisition or in systems where additional information from the environment in which the sound was acquired from is needed [1]. The information extracted from microphone array could be used for sound source localization [2][3][4], noise reduction [5][6], reverberation and echo removal [7][8][9] and much more.

Microphone arrays are also used in various smart homes and remote patient monitoring systems in order to acquire environmental and biological sounds [10][11]. One of those biological sounds is cough. Cough is a common symptom of most respiratory diseases [12][13] and hence cough detection and monitoring systems have been integrated in many patient monitoring devices [12][14][15]. 
Cough sound discrimination algorithms are part of a cough sound monitoring system whereby detected cough sounds are further classified into various cough types such as wet, dry and whooping. A novel cough sound discrimination system capable of distinguishing between dry and wet cough sounds was recently developed by [16]. The mentioned cough sound discriminator algorithms were developed and tested under ideal conditions without taking into account noise and reverberation. To be integrated into a cough monitoring system and to be used in smart homes or other patient monitoring systems, the performance of the cough sound discriminator under noisy and reverberant conditions needs to be fully analysed. Furthermore, microphone arrays and beamforming techniques could be used in order to improve the performance of the cough sound discriminator in noisy and reverberant environments.

\subsection{Problem Statement}

The cough sound discriminator developed in [16] assumed a clean cough signal free of any sources of distortion such as noise and reverberation. These sources of distortion, however, are present in the environment in which the cough sound acquisition occurs. Figure 1.1 depicts a typical cough sound acquisition setup within a patient monitoring system. As it could be seen, cough sounds are subject to noise and reverberation from the system and therefore, the performance of the cough sound discriminator might decrease as a result of this distortion. 


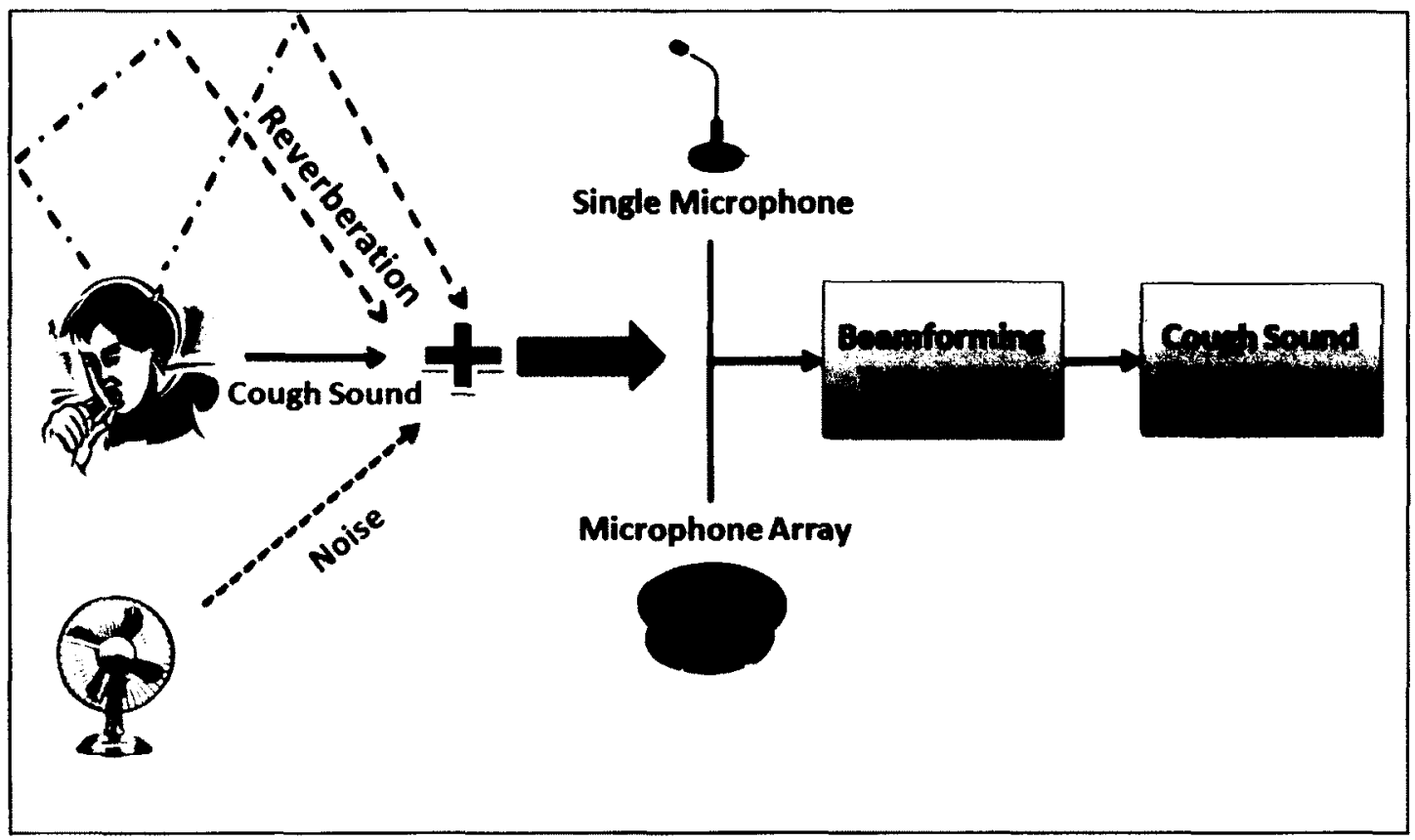

Figure 1.1: Cough sound acquisition setup.

In this thesis, the performance of the cough sound discriminator in noisy and reverberant environments is analysed. Furthermore, microphone arrays will be used in order to improve the performance of the cough sound discriminator in such environments. 


\subsection{Thesis Objectives}

The objective of this thesis is to analyse the performance of the cough sound discriminator, which was designed in [16], in noisy and reverberant environments using a single microphone for cough sound acquisition. Furthermore, a microphone array of up to 7 microphones is used along with a delay-and-sum beamforming technique in order to improve the performance of the cough sound discriminator from the single-microphone cough sound analysis. Finally, a quantitative method for measuring the performance of the cough sounds discriminator designed in [16] is introduced. In the end, a Graphical User Interface (GUI) is designed in order to help analyse the beampattern generated from the various microphone array configurations and setups.

\subsection{Thesis Contributions}

The contributions that were made during this thesis research are listed below, followed by a detailed explanation of all the contributions in the following chapters of this thesis.

Contribution 1: Designed a Linear Separation Score system using Support Vector Machines in order to quantitatively measure the performance of the cough sound discriminator under various environmental conditions. 
Contribution 2: Analysed the performance of the cough sound discriminator in a noisy and reverberant environment using a single-microphone cough sound acquisition setup. The effect of microphone-to-speaker distance on the performance of the cough sound discriminator was studied. Furthermore, the effect of the volume of the cough sounds on the performance of the cough sound discriminator was investigated under noisy and reverberant conditions.

Contribution 3: Designed microphone array and delay-and-sum beamforming technique in order to improve the performance of the cough sound discriminator under noisy and reverberant conditions. A microphone array of up to 7 microphones was used in the experimental setup. Microphone-to-speaker distance along with microphone-tomicrophone distance was varied in order to analyse their impact on the performance of the cough sound discriminator. Furthermore, the effect of cough sound volume on the performance of the cough sound discriminator was investigated using the microphone array setup in noisy and reverberant environments.

Contribution 4: Designed a Graphical User Interface (GUI) in order to study the beampattern emitted by various microphone array configurations. The GUI was capable of simulating the beampattern for any arrangements of microphone array. 


\subsection{Thesis Outline}

Chapter 2 will give a brief introduction on some of the concepts used throughout this thesis such as microphone arrays, beamforming techniques, support vector machines and cough sound discrimination. In addition, recent research done in the area of microphone arrays and beamforming will be briefly summarized.

In chapter 3, Linear Separation Score will be introduced. This score is used to quantitatively measure the performance of the cough sound discriminator under various environmental conditions.

Chapter 4 focuses on the single-microphone cough sound acquisition and the performance of the cough sound discriminator in noisy and reverberant environments using the single microphone.

Chapter 5 expands the research and experimentation performed in chapter 4 by replacing the single microphone with a microphone array. Furthermore, the beamforming technique used for processing the acquired signals from the microphone array is discussed. The performance of the cough sound discriminator is evaluated using a microphone array in noisy and reverberant environments.

In chapter 6, the Graphical User Interface (GUI) developed in this thesis will be introduced. In addition, the GUI will be used in order explain some of the results obtained in chapter 5 . 
Finally, chapter 7 will provide an overall conclusion for the research performed in this thesis followed by some suggested future work. 


\section{Chapter 2:}

\section{Background Review}

In this chapter, a thorough background and literature review is provided with regards to microphone arrays, beamforming techniques, cough sound discrimination and support vector machines.

This chapter initially provides a brief background on microphone arrays and beamforming techniques. In addition, relevant research in the area of microphone arrays and beamforming techniques will be summarized. Following the introduction on microphone arrays and beamforming techniques, the cough sound discrimination algorithm developed in [16] will be introduced.

A brief introduction on support vector machines will then be provided. Finally, the chapter will conclude with an introduction on smart homes and remote patient monitoring systems and relevant research performed in this area. 


\subsection{Microphone Arrays and Beamforming Techniques}

Microphone arrays consist of multiple microphones arranged in various arrangements in order to capture propagating sound waves. They are used in various applications such as hearing aids [17][18], speech recognition [19][20], signal detection and identification [21][22] and source localization [23]. Microphone arrays improve signal-to-noise ratio (SNR) of the acquired signals, provide sound source localization and are capable of characterizing waveforms, number of sources and their locations [24].

Microphone arrays provide spatial discrimination and filtering which can be used to discriminate the desired signal from other unwanted signals and noise [25]. Sound signals acquired by the microphones within a microphone array are fed into beamforming algorithms. Beamforming algorithms help enhance the desired signal and reduce all other interfering signals [24].

Beamforming is a spatial filter that operates on the outputs of microphone arrays or other sensor arrays in order to form a beam. There are two major steps involved in beamforming algorithms: synchronization and weight-and-sum. The synchronization step involves applying delay to the acquired signals in such a way as to align the desired signals. The weight-and-sum step involves applying "weights" to each of the inputs of the sensors within the array and adding up the results [25].

Delay-and-sum beamforming algorithm is the simplest form of beamforming algorithm. It consists of two main steps which are evident from the name of the 
algorithm: delay, followed by sum. In the delay stage of the algorithm, the signals received at the output of the sensors are time-shifted in order to align the captured signals with one another (the synchronization step). The delay is calculated based on the distance of the desired signal and the microphone array structure and the direction of arrival (DOA). In the second stage, the delayed output of each sensor is added to produce the final beamformer output. The output of the array, after applying delay to each individual microphone output, given a known source location is given by ( 1 ):

$$
y_{N}(i)=A \sum_{i=1}^{N} S\left(t-\frac{i D \cos \theta}{v}\right)
$$

where $D$ is the distance between the sensors (i.e. microphones), $N$ is the number of microphones, $\theta$ is the direction of arrival and $v$ is the speed of sound. Figure 2.1 graphically depicts the mentioned linear microphone array system.

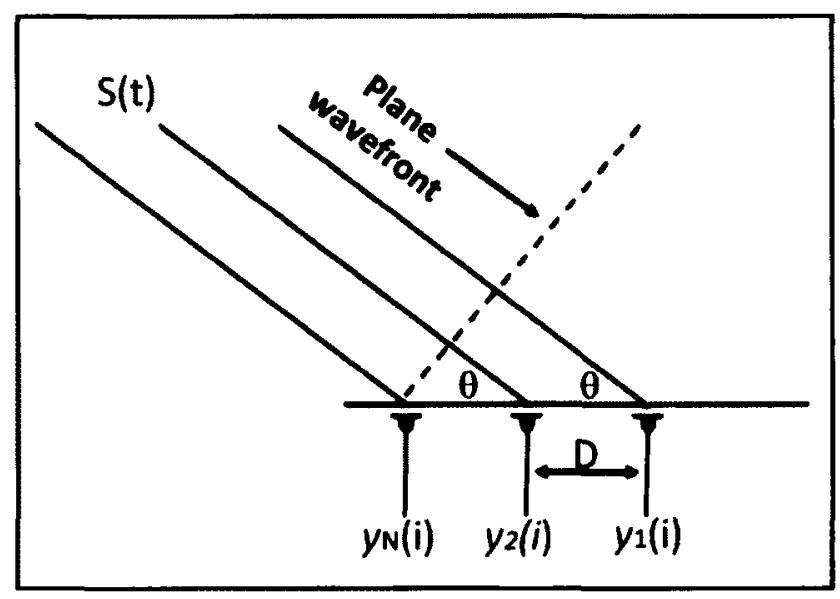

Figure 2.1: Linear microphone array arrangement. 
In the above definitions, several assumptions are made in order to simplify the beamforming analysis and calculations. The signals received at the microphones are assumed to be far-field as opposed to near-field. The difference between the two is related to the distance between the desired sound source and the microphone array. In near-field, the distance between the source and microphone array is close enough that the wave front appears curved to the microphones within the array and hence each sensor experiences a different propagation direction [26][27]. In far-field, the distance between the desired source and microphone array is far enough that the wave front reaching the microphones appears planar and hence all the microphone within the array observe the same propagation direction [28][29]. Another assumption made is the medium in which the sound wave propagates and the associated delay calculations. The sound waves are assumed to be propagating in a free-field homogenous medium and the delay calculations involve the time-delay from the source to the microphone array [30][31]. Sound waves propagating in non-homogenous fields would involve more complex calculations which take into account refraction, reflection, interference and asymmetry of the sound wave and the medium [30].

From the beamforming calculations mentioned above, it could be seen that the distance between the microphones within a microphone array, its geometry, the frequency of the desired sound source and the distance between the microphone array and the sound source play an important role in the performance of the microphone array. 
Numerous research has been performed using microphone arrays and beamforming techniques in order to reduce noise, echo and/or reverberation and improve the quality of the captured signal of interest. In the next section, recent research focused on sound acquisition (such as cough, speech) using microphone arrays is summarized.

\subsection{Sound Acquisition using Microphone Arrays}

In this section, research related to sound acquisition using microphone arrays along with the beamforming techniques used is discussed and summarized. In each of the mentioned researches, microphone arrays are used in order to improve the performance of the sound acquisition stage and enhance the desired signal.

Abu-El-Quran et al. [32] used microphone arrays and beamforming in order to perform audio classification. In their research, audio signals captured by the microphone array were classified into two high-level categories: speech and non-speech. The nonspeech sounds were further classified into more specific categories such as rain, wind, footsteps and other. The microphone array used in this research was capable of performing sound localization for the various sounds in the room and steering the microphone array beam and the cameras towards to desired sound source. The performance of the developed adaptive pitch ratio (APR) algorithm was compared to other algorithms such as linear predictive coefficients (LPC) algorithm and it was shown that the performance of the APR algorithm was greater than other algorithms used in the past [32]. 
In another study, Saruwatari et al. [33] used microphone arrays in order to improve the performance of their hands-free speech dialogue system in noisy and reverberant environments. The authors used a blind spatial subtraction array (BSSA) beamforming algorithm. In this algorithm, the signals acquired from the microphone arrays were directed to two logical paths: the primary path and reference path. The primary path consisted of the traditional delay-and-sum beamforming algorithm which was used for speech enhancement. The reference path, on the other hand, consisted of a noise estimator. The final speech was estimated using spectral subtraction procedure. As it could be seen, the performance of the speech recognition algorithm depended on the performance of the noise estimator path. The authors used an independent component analysis (ICA) based noise estimator. In this noise estimator, mutually independent output signals were produced from the observed signals without any knowledge of the room acoustics or the direction of speech. The results of the noise estimator were compared to their previously developed noise estimator which used a null beamformer and a significant improvement was observed using the ICA-based noise estimator [33].

Butko et al. [34] attempted to detect various acoustic events that occurred in a meeting room in order to describe the various activities that might be occurring. The authors made use of microphone arrays as part of their research. The microphone array was able to distinguish between different events that might occur at the same time, but from different locations. In the audio event detection (AED) system, a microphone array 
was used to provide spectro-temporal features along with audio source localization. The information extracted from the microphone arrays was further coupled with visual clues obtained from video cameras and fed into an HMM classifier in order to classify the appropriate acoustic event that had occurred [34].

In another research done by Even et al. [35] speaker activity within a small meeting room was traced using microphone arrays and scanning laser range finder (LRF). In this study, the audio signal was acquired by a circular microphone array located at the center of the meeting room. Delay-and-sum beamformer was used as part of the beamforming algorithm used in this research. The delay estimation, however, was provided to the beamformer by the LRF system. The use of LRF in speaker localization was preferred in this system since speaker localization using the microphone array was sensitive to noise and reverberation. Furthermore, the number of participants could be determined regardless of the person talking or not [35].

Martinson et al. [36] studied the relative positioning of the microphones within an array and its impact on the performance of the array in terms of sound source localization. Due to space limitation within a robot and its application in multidimensional separation of sound sources, the authors suggested using distributed microphone array structures. The distributed microphone array structures, however, produced a randomly distributed amplification pattern due to their random placement. This issue was addressed by dynamically relocating the microphones to obtain the best 
reception and localization accuracy from the region of interest using microphonemounted robots. The authors compared the results obtained using the dynamically reconfigurable microphone array structure to a randomly placed microphone array structure. The results showed a significant improvement over randomly placed microphones [36].

Thomas et al. [37] used microphone arrays and multichannel Dynamic Programming Projected Phase-Slope Algorithm (DYPSA) to improve hands-free speech recognition in noisy and reverberant environments by identifying the glottal closure instants (GCIs). The beamformer used in this study is the delay-and-sum beamforming algorithm. Due to the crucial nature of delay estimation in this algorithm, the authors used the Generalized Cross-Correlation Phase Transform (GCC-PHAT) algorithm. In this algorithm, the delay between two moderately reverberant speech signals was estimated by maximizing the cross-correlation between the two channels [37].

In another study focused on speech enhancement, Maganti et al. [38] used microphone arrays in order to enhance the acquired speech signal. The performance of the microphone array was compared to that of single-microphone speech acquisition. The speech recognition setup also included a video camera in order to track active speaker and provide relevant data to the superdirective beamformer. The results obtained from this study showed an improvement in signal-to-noise ratio enhancement and word error rate (WER) when using microphone arrays in place of a single microphone [38]. 
As it could be observed from the paragraphs above, microphone arrays are used in order to improve the quality of the desired signal in each of the mentioned studies. The goal of this thesis, however, is to improve the performance of cough sound discriminator using microphone arrays and beamforming techniques. Therefore, in the next section of this chapter, cough sound discrimination algorithms will be discussed and relevant research in this area will be presented.

\subsection{Cough Sound Discrimination}

Cough sound discrimination is a fairly new area of research. Most cough related research focus on cough detection from various other sounds such as speech [39][40][41][42]. Cough sound discrimination is the process of discriminating between different types of coughs such as wet, dry, whooping and much more [43]. Cough discrimination could be performed through either physiological properties or pathological properties [44] . Cough detection and discrimination could be performed by extracting structural information from tissue as in [45], analysing sound spectrogram and time-expanded waveform of normal and abnormal cough as in [46] or other characterization methods.

Characterizing cough sounds has the benefit of helping doctors obtain crucial information regarding the frequency, severity and nature of patient's cough. This is particularly important for patients who have difficulty describing and/or remembering their coughing episodes such as small children or elderly patients suffering from Alzheimer's disease. 
As mentioned in chapter 1 , the cough sound discrimination algorithm developed by [16] is the main focus of this thesis. As a result, the research performed in [16] will be summarized in greater detail in the proceeding paragraphs of this section.

Chatrzarrin et al. designed two features, i.e. Feature 1 and Feature 2 in order to discriminate between dry and wet cough sounds. In this research, a cough signal was divided into three phases: phase 1, 2 and 3 based on the cough analysis outlined in [47]. The first feature, Feature 1, was a time-domain feature and it was based on the number of peaks of the energy envelope of the cough signal. Research revealed that wet coughs in general have more peaks than dry coughs and this finding was used as the first basis of discrimination between dry and wet cough sounds (Figure 2.2). The second feature, Feature 2, which was a frequency-domain feature, was based on the power ratio of two frequency bands of the second phase of the cough signal. Research had shown that wet coughs exhibit a large power peak in the $[0-750 \mathrm{~Hz}]$ frequency band. Dry coughs, on the other hand, exhibit the same peak in the [1500-2250 Hz] frequency band. This difference was used as another source of discrimination between dry and wet cough sounds (Figure 2.3). Finally, the cough sound discriminator algorithm was tested on a database of 17 highly dry and highly wet cough sounds and the result of the algorithm is shown in Figure $2.4[16]$. 


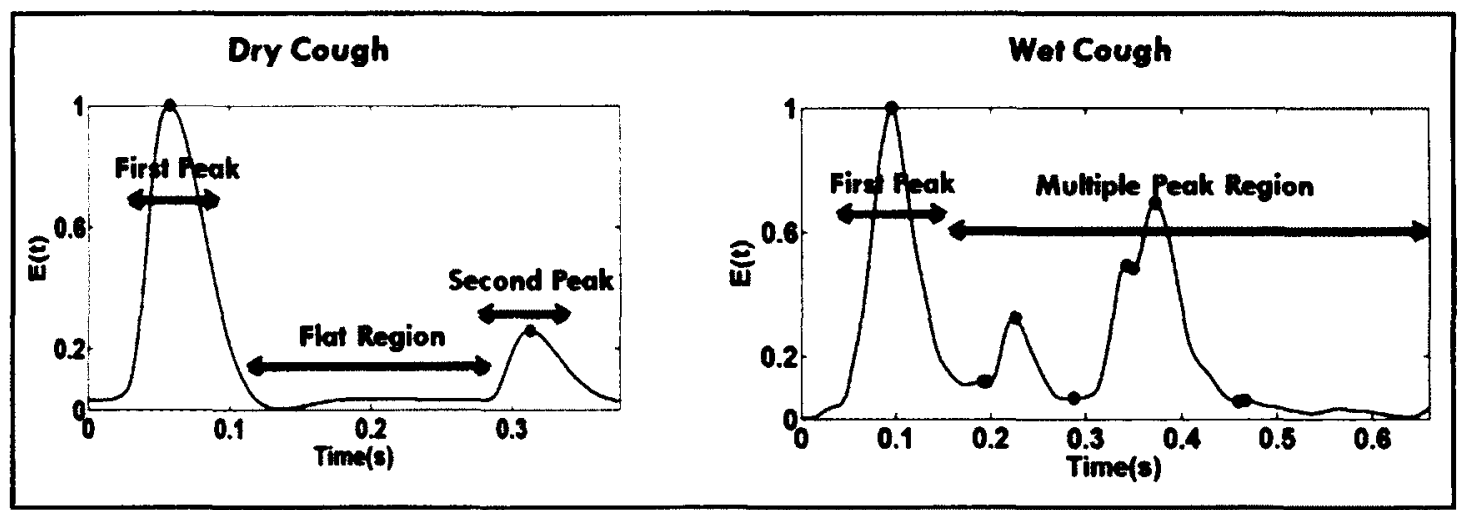

Figure 2.2: Feature 1 of cough sound discriminator (Taken with permission from [16]).

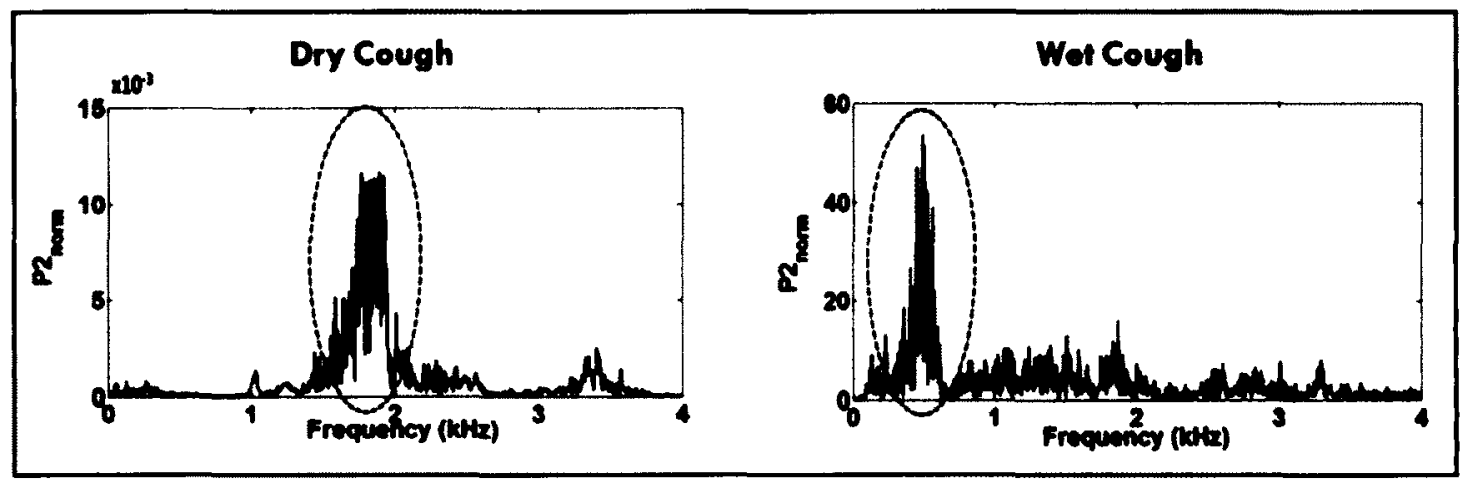

Figure 2.3: Feature 2 of cough sound discriminator (Taken with permission from [16]). 


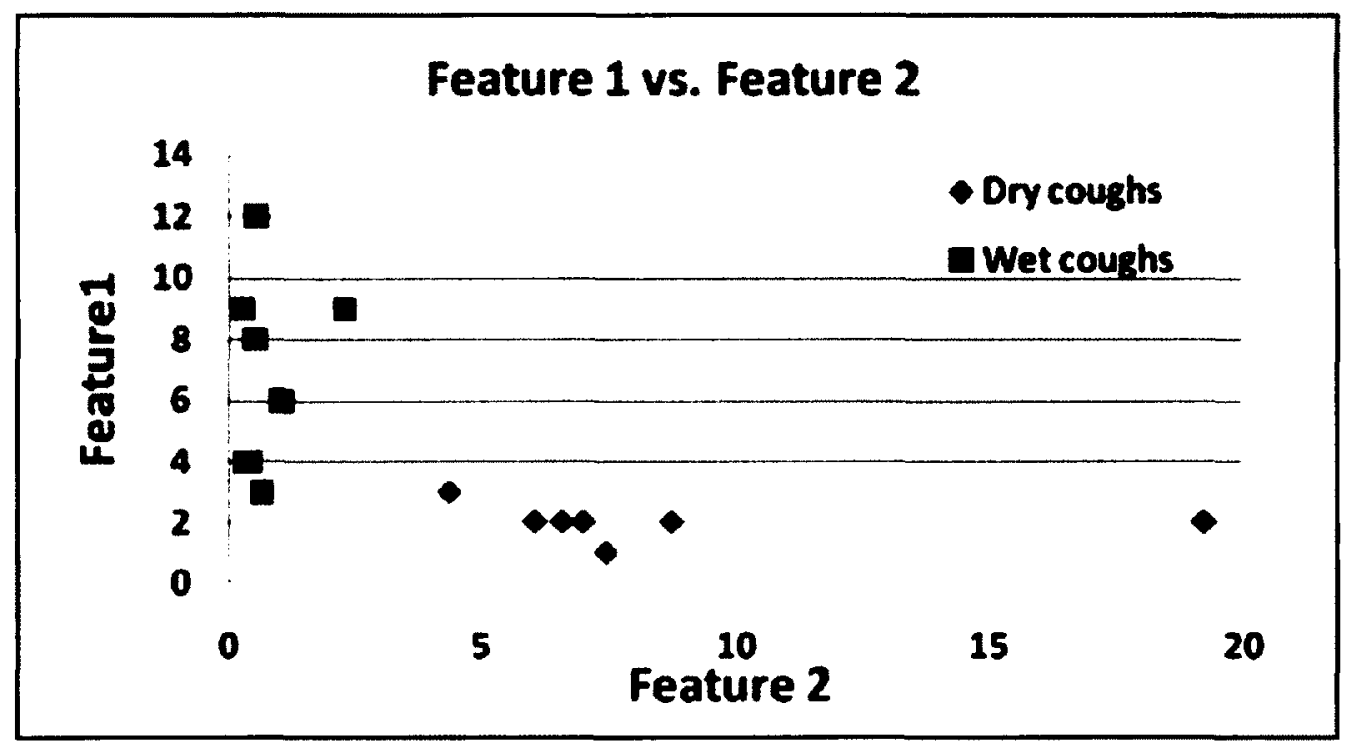

Figure 2.4: Cough sound discriminator result (Taken with permission from [16]).

\subsection{Support Vector Machines}

In this section, a brief introduction to Support Vector Machines (SVM) is provided since it is used as a mathematical tool in this thesis.

Support vector machines (SVMs) are capable of separating data belonging to different classes by finding a hyperplane which separates the classes from one another. Although this characteristic is typical of other linear discriminants, the hyperplane selected by an SVM has the maximum distance to the support vectors. Therefore, the hyperplane is considered to have the maximum margin [48][49]. The equation of the hyperplane is depicted in (2) and the equation for maximizing the distance between the 
hyperplane and the support vectors is shown is (3). Figure 2.5 depicts a typical support vector machine and the separating hyperplane.

$$
\begin{gathered}
\pi_{\omega, b}=\omega \cdot x+b=0, x \in R^{n} \\
\max (\omega, b)\left(\min _{i} d\left(\pi_{\omega, b}, x_{i}\right)\right)
\end{gathered}
$$

where $\pi$ is the hyperplane, $\omega$ and $b$ are the slope and y-intercept of the hyperplane, $d$ is the distance between the hyperplane and data points $x$.

In order to solve the above equations and obtain an equation for the hyperplane, two set of constraints need to be addressed as shown in (4). It turns out only a subset of the data points will meet these constraints. These data points are named "support vectors" and are depicted by blue circles in Figure 2.5 [48].

$$
\min _{\omega, b} \frac{1}{2}\|\omega\|^{2}
$$

$$
\text { subject to } y_{i}\left(\omega^{T} x_{i}+b\right) \geq 1
$$




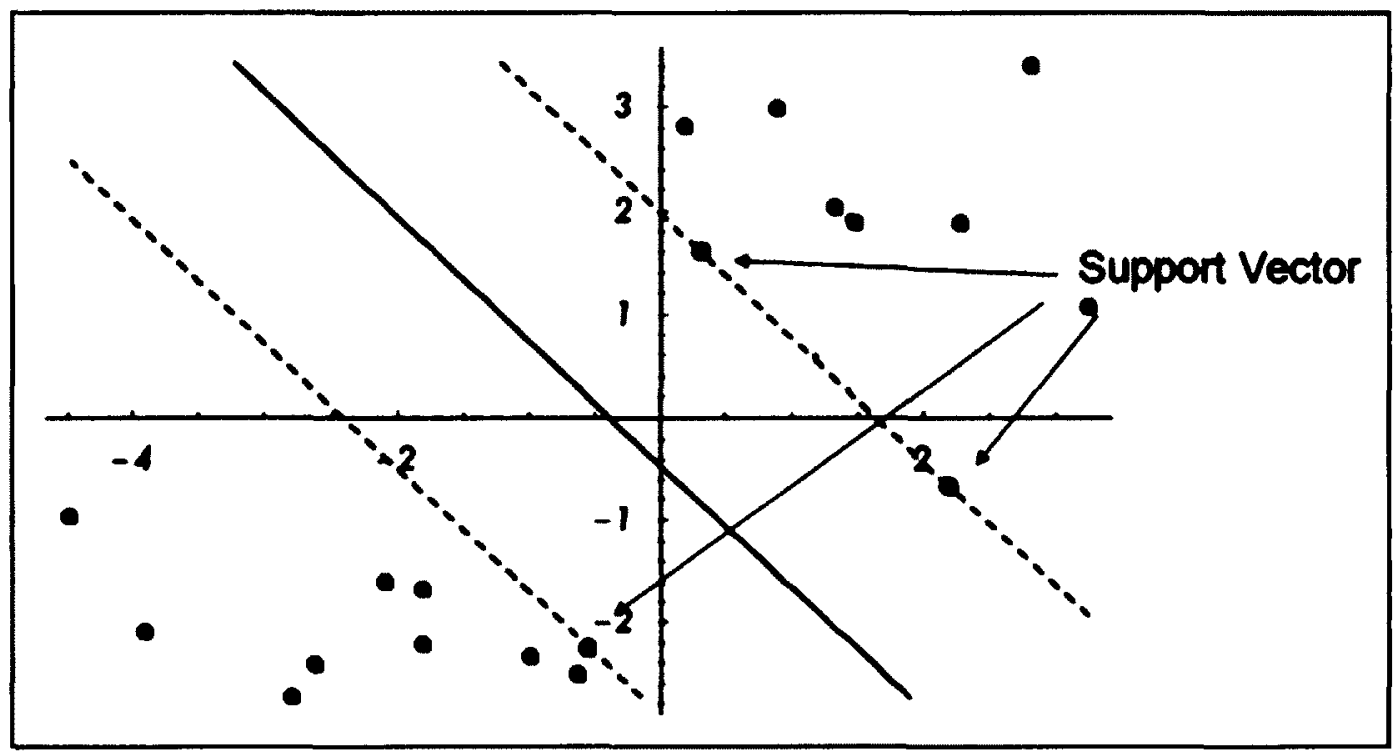

Figure 2.5: Support Vector Machine.

When training a support vector machine, the hyperplane may not necessarily be linear. Support vector machines include a kernel which is used for mapping the input space into a potentially higher dimensional space. There are different types of kernels such as linear, polynomial, radial basis, three-layer neural network and much more [48].

Support vector machines are used in this thesis as a mathematical tool in order to draw the best separating line between the dry and wet cough sounds.

\subsection{Remote Patient Monitoring}

Smart home technologies and remote patient monitoring have gained a lot of attention in recent years due to an increase in the aging population and the cost savings and convenience associated with remote patient monitoring [50][51][52][53][54][55][56]. 
Microphone arrays play a critical role in most remote patient monitoring systems for various audio signal capturing purposes. In this section, relevant research on the use of microphone arrays in smart homes or remote patient monitoring devices is explained and summarized.

Popescu et al. [57] used microphone arrays in order to detect falling sounds of patients within a smart home. In this study, microphone arrays along with motion detectors were used in order to detect fall sounds. The motion detectors were used in order to detect whether a patient was moving or not, which could help verify whether a fall reported by the microphone array algorithm was possible or not. If the detected motion by the motion detector and the detected fall sound by the microphone array occurred within a certain time window, the caregiver would get notified [57].

Guettari et al. [58] investigated the use of Smart Home Person Tracking (SHPT) and Audio Person Tracking (APT) in order to improve the precision of localization. The SHPT used infrared sensors, whereas the APT used microphone arrays. The microphone arrays were capable of estimating the direction of arrival (DOA) of a given sound source with a reliable precision of $15^{\circ}[58]$

In another study, Axis et al. [10] describe various technologies and smart clothing used for patients and smart homes. In this paper, a "citizen medicine" structure is described whereby patients help monitor their health conditions at home or anywhere they go. The technology used an intelligent clothing which was equipped with numerous 
sensors each responsible for monitoring a certain type of biological signal. In addition to smart clothing, the home of the patient was also equipped with various sensors such as video movement detectors, IR sensors, contact sensors, weight sensors and microphone arrays. The microphone arrays were used for purposes of patient localization and for detecting various sounds (such as fall) [10].

Arcelus et al. [11] describe the integration of various smart home technologies in a health monitoring system for the elderly. In this paper, a home equipped with numerous sensors is described. Each of the intelligent sensors described in this paper are used to monitor a specific activity of the patient or monitor a specific vital signal from the patient. In this home, magnetic switches were used for monitoring entry and exit of patients from various rooms, thermistors were used to provide temperature for various appliances such as stove or water, accelerometers were used to detect high impacts such as falling or sitting too fast on a chair, radio frequency identification (RFID) was used to track the location of various devices (such as television remote control) and much more. Microphone arrays were also used in the smart home as a way of monitoring sound activities in the room and also providing useful information which could be used for detecting abnormal noises or calls for help [11].

\subsection{Conclusions}

In this chapter, relevant research related to microphone arrays and beamforming techniques was summarized. Furthermore, a cough sound discrimination algorithm was 
discussed along with microphone array technologies used in smart homes and remote patient monitoring systems. In the following chapters, microphone arrays will be used in order to improve the performance of the cough sound discriminator in noisy and reverberant environments. 


\section{Chapter 3:}

\section{Cough Sound Discriminator Performance}

The cough sound discriminator discussed in [16] is capable of distinguishing between dry and wet cough sounds using two unique feature extraction algorithms. The cough sounds used in developing and testing the cough sound discriminator, however, were free of any source of distortion such as noise and reverberation. Under these ideal conditions, the discriminator was capable of distinguishing between dry and wet coughs sounds with an accuracy of $100 \%$. This accuracy was visually confirmed by plotting Feature 1 versus Feature 2 of the coughs sound discriminator and noticing an obvious separation between the two types of coughs [16].

The ideal condition assumed in the design of the cough sound discriminator was a necessary first step in designing the feature extraction algorithms. Those ideal conditions, however, do not represent the environment in which cough sounds are typically acquired from. Cough sounds acquired from patients using microphones will be distorted by noise 
and reverberation from the patients' environment and therefore, the performance of the cough sound discriminator might be affected. In this chapter, a quantitative method of measuring the performance of the cough sound discriminator is introduced and discussed in detail.

\subsection{Cough Sound Database}

In order to analyse the performance of the cough sound discriminator, the complete cough sound database used in [16] was used. The databases consisted of 46 cough sounds (19 dry and 27 wet cough sounds). The cough database was obtained via online sources and local cough sound recordings.

The nature of the cough signals obtained from [16] was verified by medical professionals. Each of the cough sounds within the database contained a single cough sound as opposed to multiple and repeated cough sounds.

\subsection{Linear Separability of Cough Sound Discriminator}

In order to quantify the performance of the cough sound discriminator, the linear separability between the dry and wet cough sounds discriminated by the cough sound discriminator is considered. Figure 3.1 depicts the cough sound discriminator result under ideal conditions. As it could be seen from the figure, dry and wet cough sounds could be completely separated with a straight line. It is expected that as the performance of the cough sound discriminator decreases, the dry and wet cough data will get closer to one 
another and start mixing. In these situations, it would be difficult, if not impossible, to linearly separate the two cough types from one another.

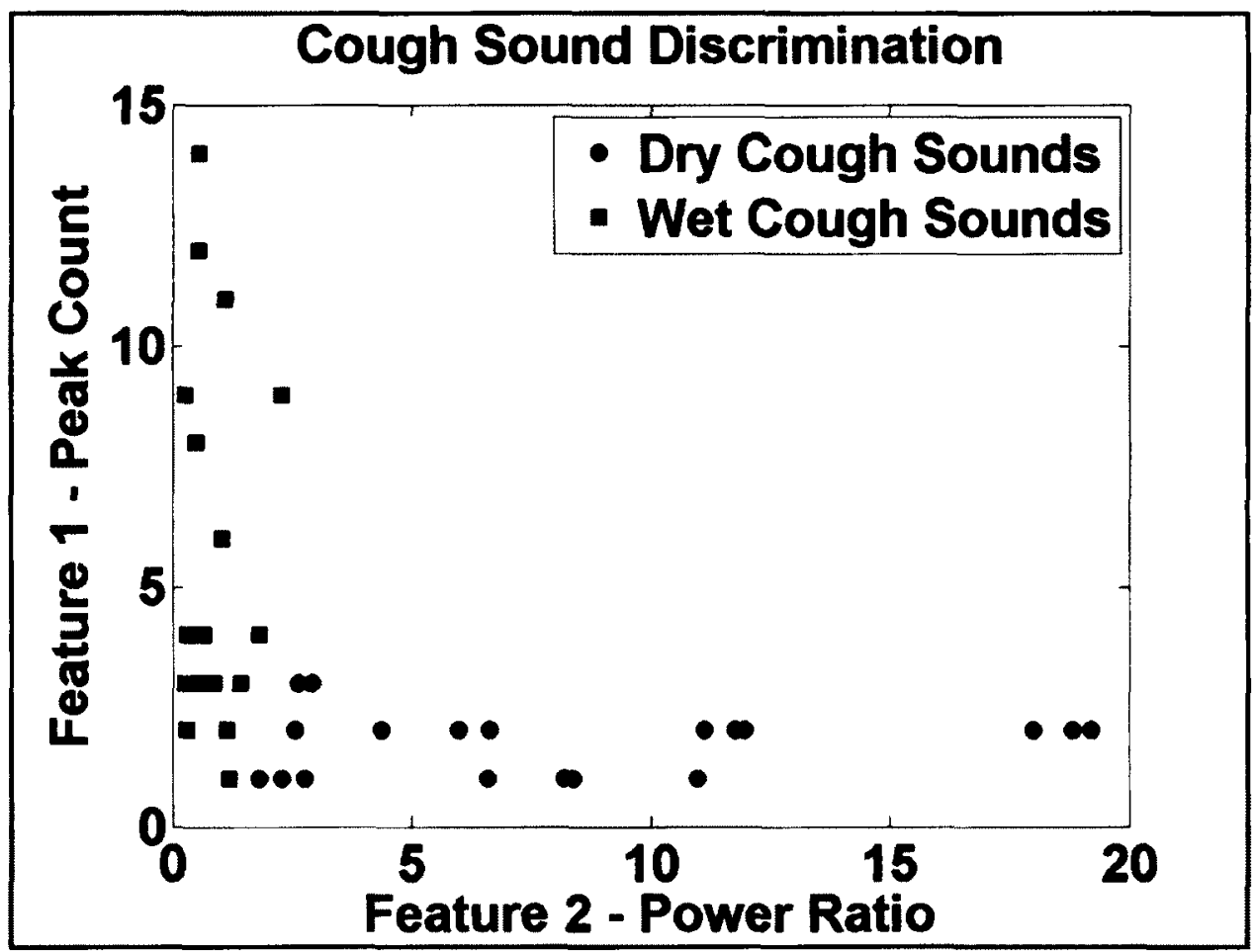

Figure 3.1: Cough sound discrimination.

Although it is possible to manually draw the line which separates the two cough sounds from one another, drawing the separation line manually is not preferred. Manually drawing the separation line will introduce inconsistencies in the method used to draw the line. Furthermore, manually separating the two cough types would eliminate any possibilities of automating this process and integrating it into a patient monitoring system. In order to mathematically draw the separation line and introduce a way of 
automating this step, support vector machines are used and discussed in the following section.

\subsection{Linear Separation using Support Vector Machines}

As discussed in section 2.4, support vector machines, SVMs, could be used in order to

find the hyperplane which separates dry and wet cough sounds. The advantage of using SVM as opposed to other linear discriminants is the fact that the hyperplane found by an SVM has the largest distance to the closest data point [59].

In this research, the SVM was only used as a mathematical tool capable of drawing a line which could separate the two cough types from one another. In order to train the SVM to draw the best line of separation, SVM classifier was trained using the entire cough database used in [16]. By training the classifier with all the data from the database, the classifier becomes over-trained and is capable of successfully separating the cough types, which is the goal of this research [60].

In order to design a support vector machine capable of separating dry and wet cough sounds in a linear fashion, an SVM with a linear kernel was used. Furthermore, in order to overfit the classifier and ensure an actual separation line is computed, the SVM classifier was designed with a hard margin. An SVM trained with hard margins instead of soft margins results in an SVM with minimal tolerance for outliers. As a result, the SVM becomes overfitted [61]. Hard margins and soft margins in an SVM define the sensitivity of the SVM for outliers. The constraint equation introduced in (4) is an example of a hard 
margin support vector machines. In order to introduce soft margins, a slack variable, $\xi$, is added to (4) as shown in (5):

$$
\min \frac{1}{2}\|\omega\|^{2}+c \sum \xi
$$

The higher the value of the constant $C$, the more sensitive the SVM becomes and hence the SVM becomes hard-margin [61].

The SVM classifier for this thesis was designed using Matlab bioinformatics toolbox as described in [62].

\subsection{Linear Separation Score}

The SVM classifier mentioned in the previous section was overfitted with a hard margin in order to ensure the best separation line is computed by the SVM classifier. The "best" separation line is defined as the line in which the least amount of data is misclassified. In order to further quantify the performance of the classifier, the percentage accuracy or the Linear Separation Score (LSS) was used as depicted in (6).

$$
L S S=\left[\frac{\text { correctly classified samples }}{\text { All samples }}\right] \times 100
$$

Using the LSS defined in (6), the linear separability of the dry and wet cough sounds as determined by the cough discriminator could be determined. This score is used in the proceeding sections of this thesis in order to evaluate the performance of the cough sound discriminator under various environmental conditions. From the definition of LSS, it 
could be concluded that the higher the value of LSS, the better the performance of the discriminator, up to a maximum value of 1 . The LSS of the ideal case is computed to be 1 as it could be seen from Figure 3.2.

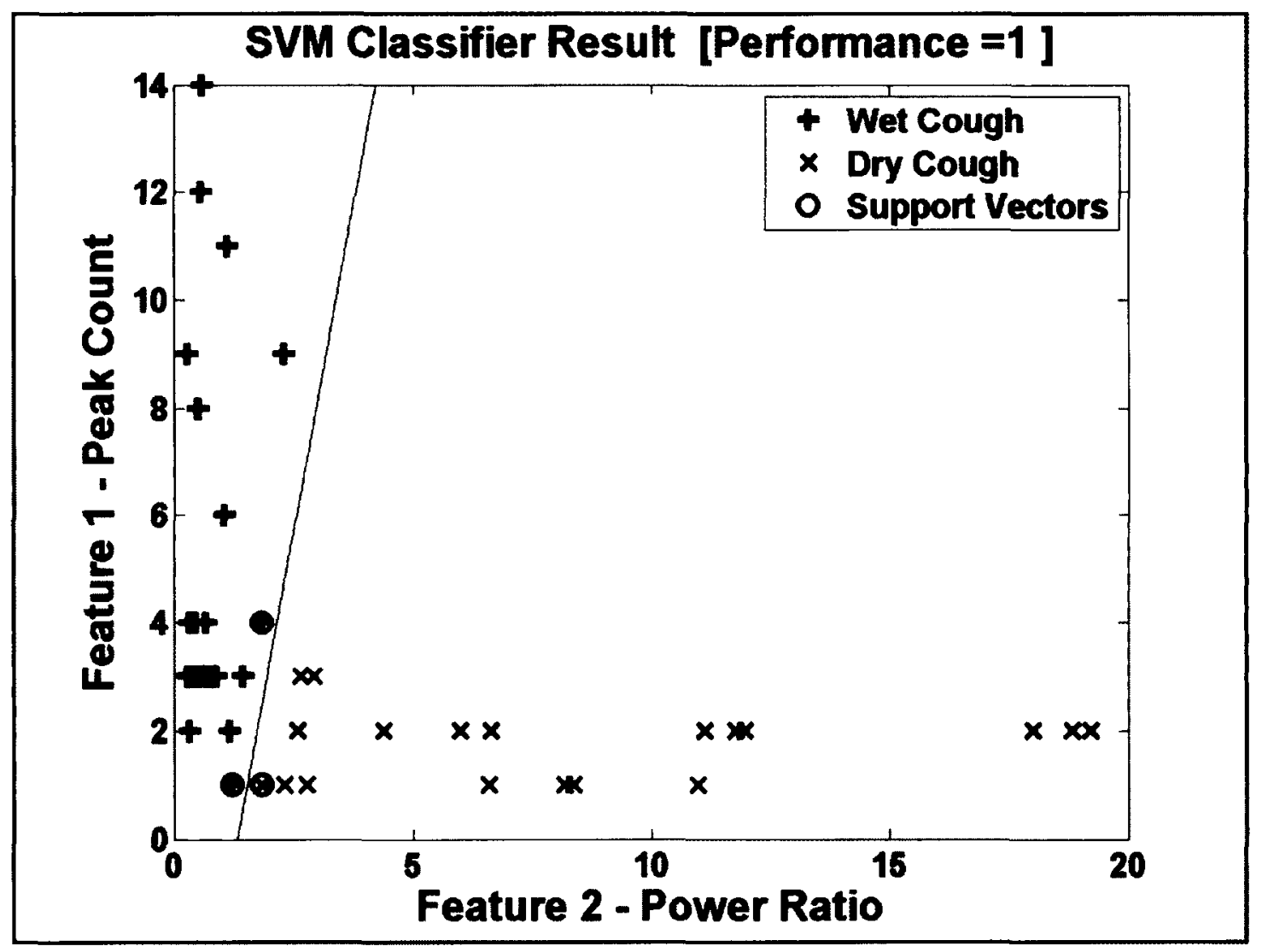

Figure 3.2: SVM classifier result.

Figure 3.3 summarizes the overall system used in order to evaluate the performance of the cough sound discriminator. Cough sounds from various experimental setups are 
passed through the cough sound discriminator system and the computed features are passed through the SVM classifier in order to compute the Linear Separation Score of the discriminator.

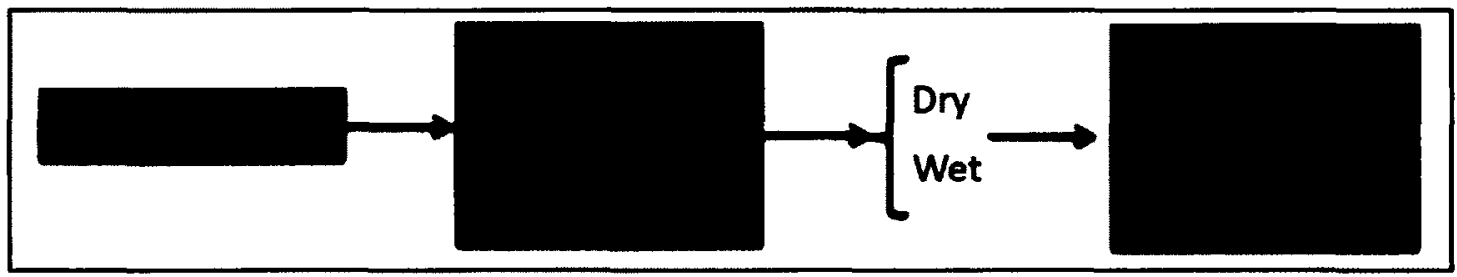

Figure 3.3: Linear Separation Score system.

\subsection{Discussion}

In this research, Linear Separation Score was used in order to quantify the performance of the cough sound discriminator. The LSS is the classification accuracy of the SVM. The reason for renaming the classification accuracy to LSS in this research was to avoid confusions that might result due to the use of the term "classification accuracy".

As mentioned previously, SVM was used as a mathematical tool in this research and it was not used as an actual classifier. Using the term "classification accuracy" might confuse the reader in thinking that an actual classification was performed. Therefore it was decided to use the term Linear Separation Score instead of classification accuracy.

In order to obtain the Linear Separation Score, the entire database was trained and classified. Alternative methods would have involved using various cross-validation techniques such as leave-one-out procedure. Using the entire database for both training 
and classification resulted in consistent results for the LSS of a given experimental setup. Using other techniques such as leave-one-out cross-validation would result in different scores for a given experimental setup. The reason for this variance is the fact that different groups of data would be selected for training and classification and depending on the selected data, the SVM would result in a different separation line, hence resulting in a different LSS. Although this variance is expected, it is not an acceptable result for the purposes of this research. In this research, numerous experimentations were performed using actual hardware and obtaining different results for the same experimental setup would make it difficult to identify the source of variance in the setup. Obtaining a different LSS for an identical setup could either be the result of an experimental flaw, which needs to be addressed, or the expected result of the cross-validation technique. In order to avoid such confusion, cross-validation was not used in measuring the performance of the cough sound discriminator.

The Linear Separation Score was used as the quantitative measure of the performance of the classifier. An alternative method would have been to use the margin obtained from the SVM. As mentioned previously, the margin is defined as the distance between the hyperplane and the support vectors. Using the margin could provide the degree of separability for a given result. Two experimental results could both result in an LSS of 1; however, one might have a larger margin than the other which translates into one being more separated than the other. LSS is a good first step in quantitatively measuring the 
performance of the cough sound discriminator. Incorporating the margin in future research provides a more quantitative measure of the performance of the cough sound discriminator.

\subsection{Conclusions}

The performance of the cough sound discriminator was evaluated using the linear separability of the dry and wet cough sounds. An SVM classifier was used in order to mathematically compute the separation line between the two cough types. In order to ensure the SVM computes the most accurate separation line, it was overfitted by using all the cough data in both the training and classification steps along with using a hard-margin as opposed to a soft-margin for this classifier. In addition, a Linear Separation Score (LSS) was used in order to quantitatively measure the performance of the cough discriminator. 


\section{Chapter 4:}

\section{Cough Sound Acquisition using a Single}

\section{Microphone}

In this section of the thesis, the performance of the cough sound discriminator is studied in reverberant and noisy environments. This analysis provides a realistic view of the performance of the cough sound discriminator in a typical environment where the actual cough sound monitoring and recording might occur. The chapter starts off with a detailed description of the experimental setup, followed by the performed experimentations and results and conclusions.

\subsection{Experimental Setup}

The experimental setup used for single-microphone sound acquisition consisted of numerous hardware and software components which are summarized in Table 1. 
Table 1: Cough sound acquisition experimental setup

\begin{tabular}{|l|l|}
\hline Carleton University DSB Lab & Sound acquisition environment \\
\hline Dell Vostro 430, IntelB Core i7 & Main PC \\
\hline MOTU 896HD & Hardware used for playback and capture \\
\hline SAMSON SERVO-150 & Amplifier \\
\hline Audio-Technica AT803B & Microphone \\
\hline KOSS M85 Plus & Speaker \\
\hline Neutrik XLR Cable & Audio Cable \\
\hline Firewire Cable & MOTU to PC Connection \\
\hline Adobe Audition 3.0 & Software used for playback and capture \\
\hline Cough Database & Database of dry and wet cough sounds \\
\hline
\end{tabular}

The cough acquisition experiment using a single microphone was carried out at Carleton University Digital Signal Processing (DSP) Lab, which is a relatively large room of dimensions $11 \mathrm{~m} \times 10 \mathrm{~m} \times 4 \mathrm{~m}$. The room contained tables, chairs, glass, and all other typical office equipment. This room was selected because all sound acquisition hardware and software were located in it. Furthermore, the room provided a typical reverberant environment where cough sound acquisition may occur.

MOTU-896HD, Figure 4.1, was used for sound playback and capture. This hardware device consisted of $824-b i t 192 \mathrm{kHz}$ analog inputs and 2 analog outputs. The power of 
all input and output ports were tuneable which could be used in order to prevent output saturation and input data clipping.

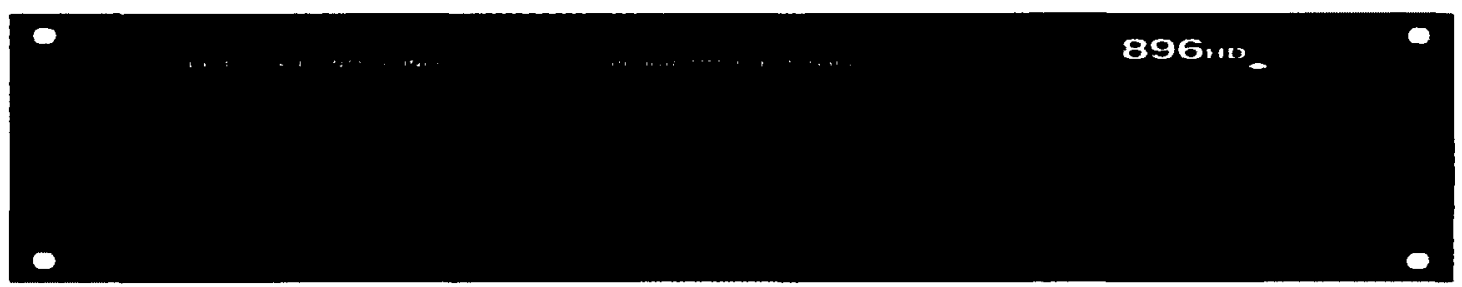

Figure 4.1: MOTU-896HD.

SAMSON Servo-150, Figure 4.2, was used as an optional amplifier for sound sources with very low volume. It was also used to provide a wider volume range for various experiments conducted in this research.

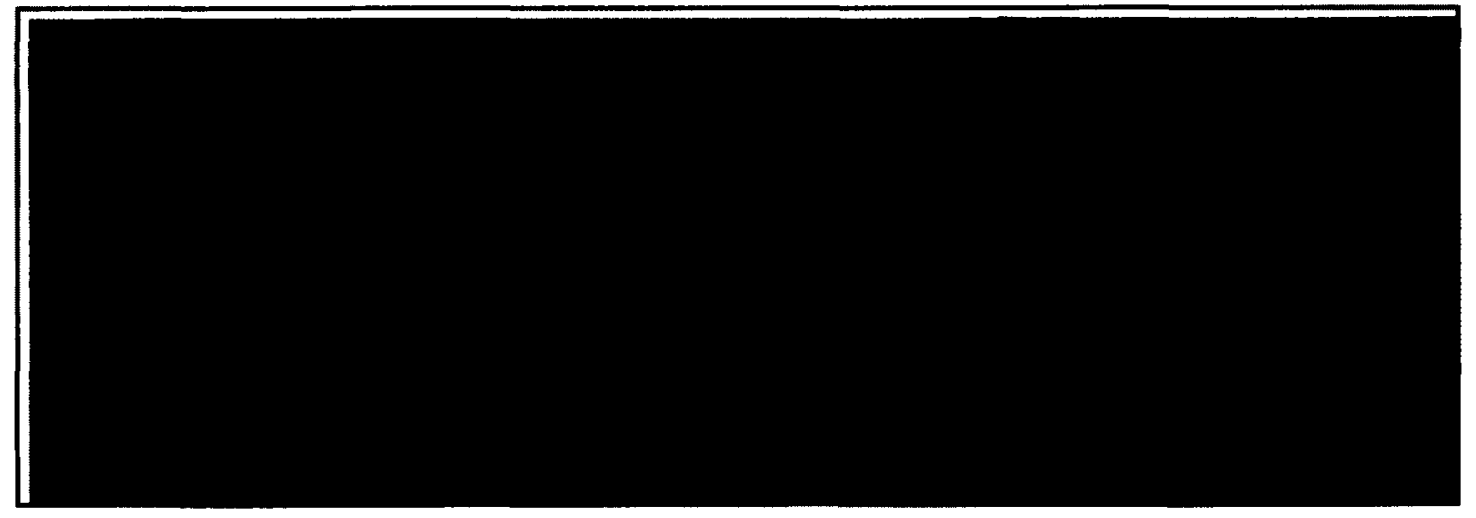

Figure 4.2: SAMSON SERVO-150. 
Audio-Technica AT0803b microphones, Figure 4.3, were used to capture sound signals. The microphone came equipped with its own battery-powered power module. In addition, the microphone was capable of switching from flat frequency response to a lowend roll-off frequency response mode.

KOSS M85 Plus speaker, Figure 4.4, was used to play various sound signals emitted by either the MOTU audio interface or the SAMSON amplifier.

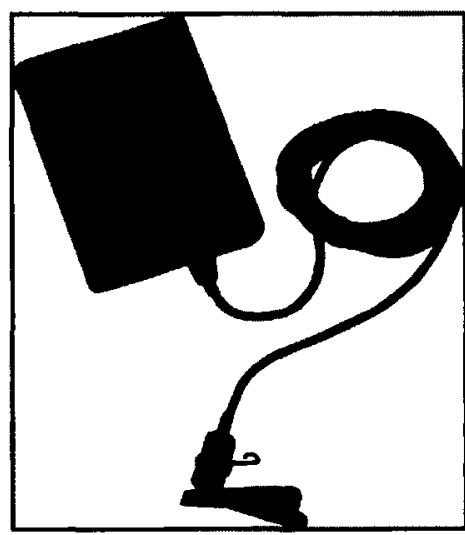

Figure 4.3: Audio-Technica AT803B.

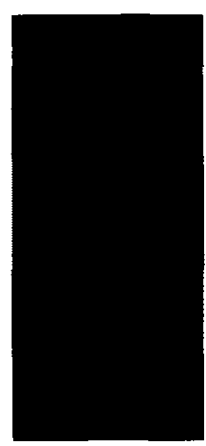

Figure 4.4: KOSS M85 Plus. 
Finally, Neutrik XLR Cables, as shown in Figure 4.5, were used to connect the Audio-Technica AT0803b microphones to the MOTU audio interface. The MOTU audio interface, on the other hand, was connected through a firewire connection, Figure 4.6, to the main $\mathrm{PC}$.

The cough sound database used in the cough sound acquisition experiments was the same database used in [16] which consisted of 19 dry and 27 wet cough sounds. This was done in order ensure consistent comparison between the cough sound discriminator described in [16] and its performance in reverberant and noisy environments.

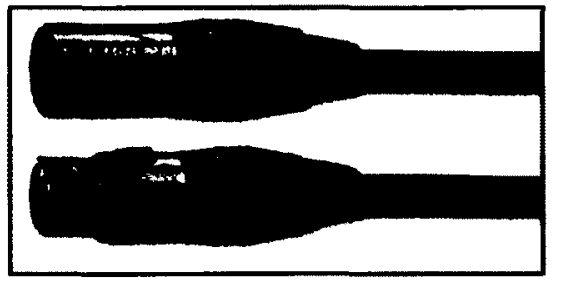

Figure 4.5: Neutrik XLR Cable.

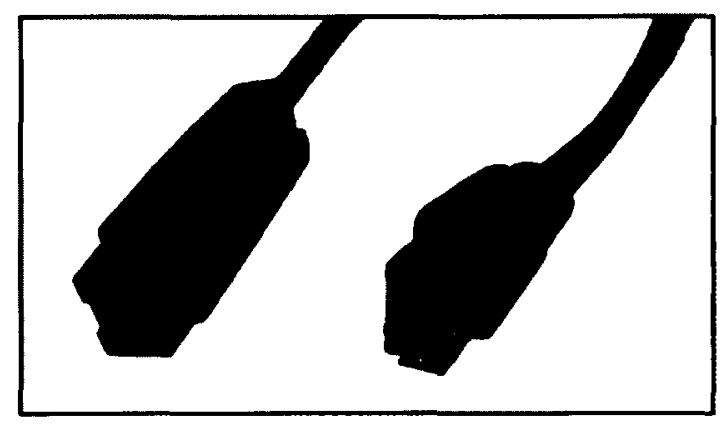

Figure 4.6: Firewire Cable. 
The overall experimental setup consisted of 1 or more microphones clipped onto a stand. The microphones were connected through their power modules to an XLR cable and routed to the back of the MOTU audio. A typical recording session where a sound wave is played through the speaker and captured by the microphone is depicted in Figure 4.7.

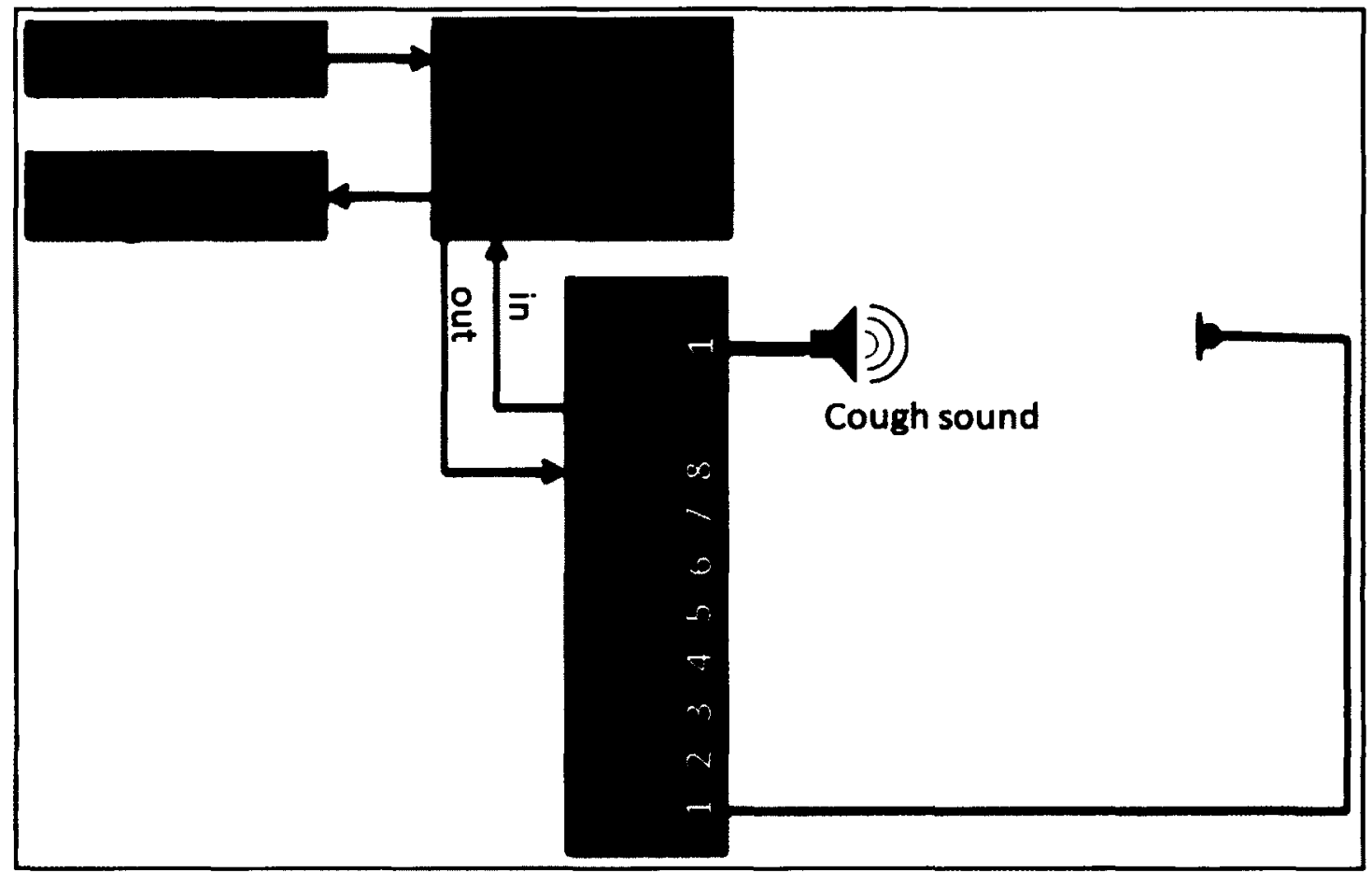

Figure 4.7: Single cough sound acquisition setup.

Adobe Audition 3.0 was used for playing and capturing various sound signals through the MOTU audio interface. Adobe Audition has the capability of monitoring sound levels, modifying input and output volume levels and playing and capturing one or more 
sounds simultaneously. All sound signals were sampled at $44.1 \mathrm{kHz}$ which was the lowest sampling frequency supported by the MOTU audio interface.

With the experimental setup described above, numerous cough sound acquisition experimentations were performed in order to analyse the performance of the cough sound discriminator in reverberant and noisy environments.

In order to ensure the validity of the experimental setup and to understand the limitations of the various devices in the system, a loopback experiment was performed and will be described in great detail in the next section.

\subsection{Loopback Experiment}

Section 4.1 described the overall experimental setup used for the purposes of evaluating the performance of the cough sound discriminator in reverberant and noisy environments. Before performing detailed analysis on the performance of the cough sound discriminator, however, the validity of the experimental setup needed to be confirmed. For this reason, a loopback experiment was performed.

Loopback experiment setup is shown in Figure 4.8. As it could be seen from the figure, the output of the MOTU audio interface was routed to one of its analog inputs. Cough sounds were played and captured by Adobe Audition software. In this experimental setup, the cough sounds were distorted by the inherent limitations of Adobe Audition software, MOTU hardware and the connecting cables between the output and input port, in addition to the firewire connection between the PC and the audio interface. 
The entire cough database was passed through the system depicted in Figure 4.8 and the recorded cough sounds were passed through the Linear Separation Score system shown in Figure 3.3.

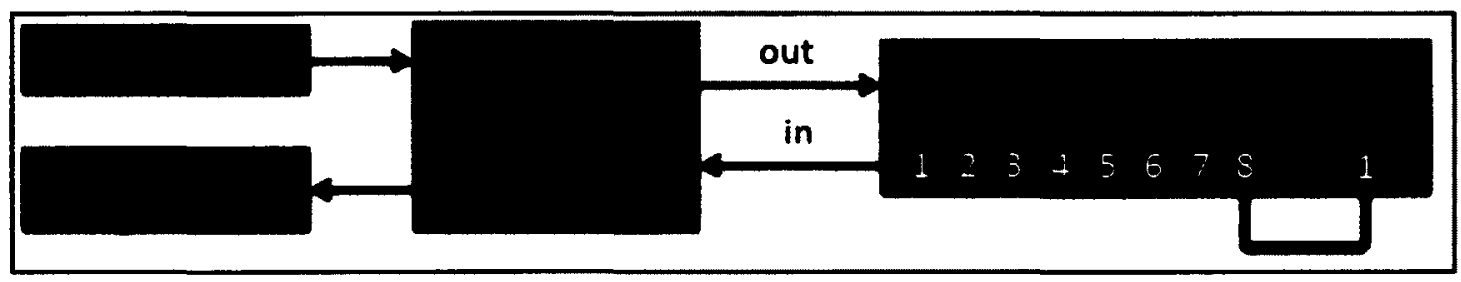

Figure 4.8: Loopback experimental setup.

The Linear Separation Score achieved by the loopback experiment is shown in Figure 4.9. From the figure, it could be seen that the loopback experiment also resulted in a Linear Separation Score of 1.

In order to further confirm the validity of the experimental setup, the output of the cough sound discriminator between the ideal condition depicted in Figure 3.2 and the loopback experiment depicted in Figure 4.9 was compared and as it could be seen, with the exception of one or two cough samples, the results are very similar to one another.

These results confirm the validity of the experimental setup. They also confirm that the inherent noise and non-linearities within the system do not distort the cough sounds in such a way that it would affect the performance of the cough sound discriminator. 
The single-microphone cough sound acquisition was performed once the validity of the system was confirmed and an LSS of 1 was obtained. The obtained LSS was the same score obtained when measuring the performance of the cough sound discriminator under ideal conditions. The details of the single-microphone cough sound acquisition are explained in great detail in the next section of this chapter.

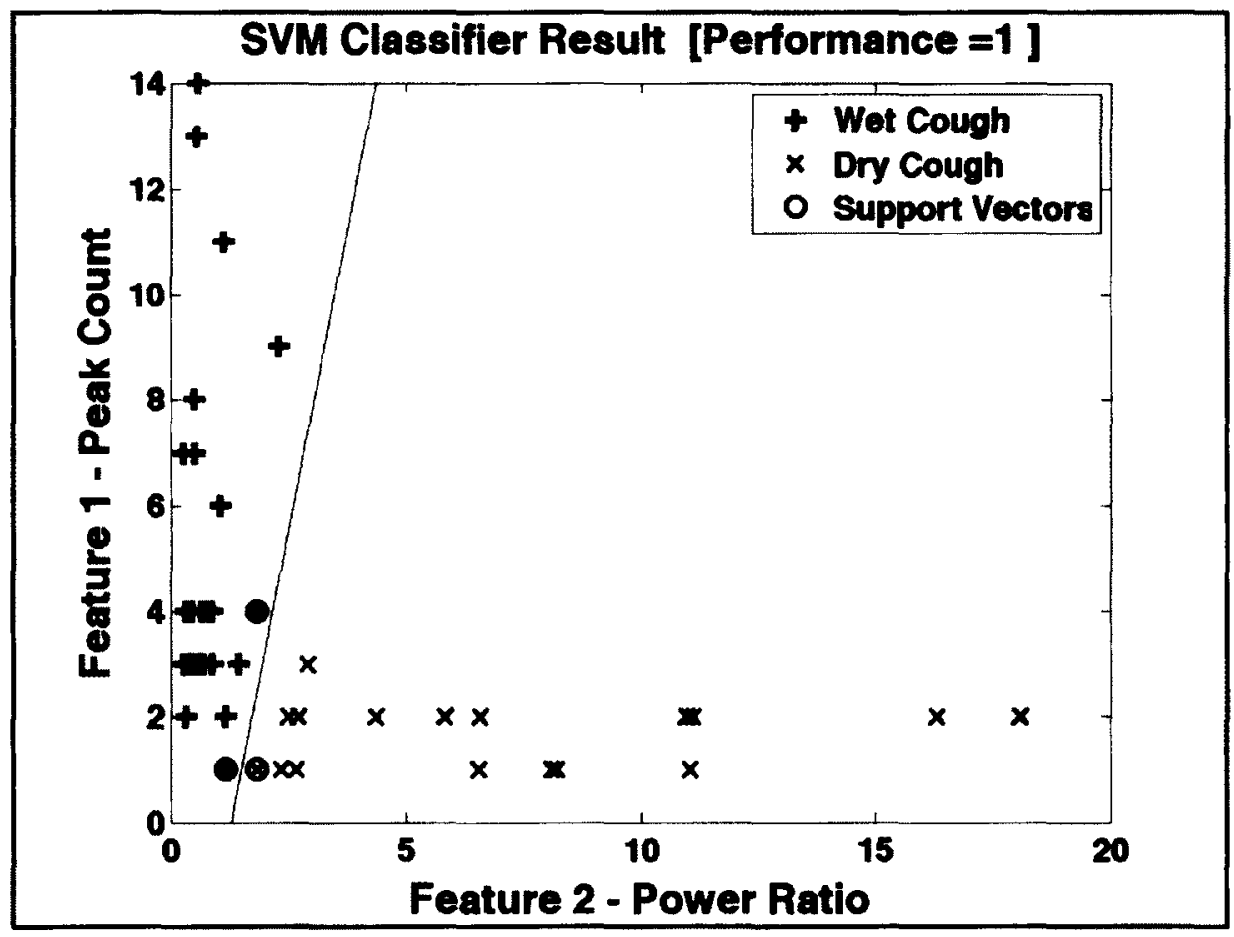

Figure 4.9: LSS of the loopback experiment. 


\subsection{Effect of Reverberation}

The performance of the cough sound discriminator was evaluated in both the ideal case (Figure 3.2) and the loopback experiment (Figure 4.9). Although in both cases, a complete linear separation between the identified dry and wet cough sounds was observed, the results are not indicative of the performance of the discriminator in reverberant and noisy environments. It is expected that the performance of the cough sound discriminator will decrease when cough sounds become distorted by noise and reverberation in the room.

In the first part of the single-microphone experimentation, the performance of the cough sound discriminator is analysed by capturing cough sounds with a single microphone in a reverberant room.

A single microphone is located directly in front of the speaker similar to Figure 4.7. Cough sounds are played through the speaker via the output of the MOTU audio interface and captured through the analog input of the MOTU device. Similar to the loopback experiment, the recorded cough sounds are passed through the cough discriminator system and the performance of the discriminator is determined using the Linear Separation Score. The volume level at the output of the Adobe Audition software was set to $-10 \mathrm{~dB}$. This level was chosen since it did not cause output saturation in any of the played cough sounds. 
In the single microphone cough sound acquisition experiment, the distance between the microphone and the speaker is varied in order to determine the effect of distance between the patient emitting the cough and the microphone capturing it. A total of 5 experiments were performed in which the following speaker-to-microphone distances were used: $50 \mathrm{~cm}, 100 \mathrm{~cm}, 150 \mathrm{~cm}, 200 \mathrm{~cm}$ and $250 \mathrm{~cm}$.

Figure 4.10, Figure 4.11, Figure 4.12, Figure 4.13 and Figure 4.14 show the performance of the cough sound discriminator in terms of its Linear Separation Score (LSS). As it could be seen from the graphs, the performance of the cough sound discriminator decreases as the distance between the speaker and the microphone is increased. Table 2 summarizes the results obtained from the single microphone cough sound acquisition experiment. 


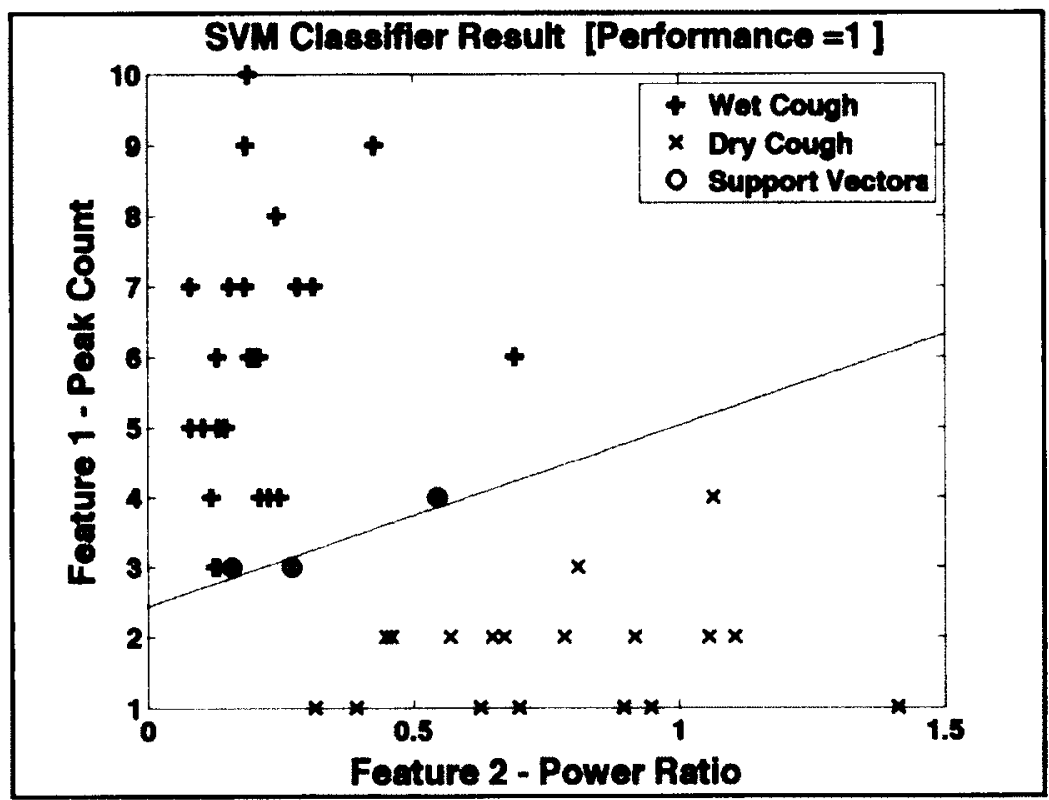

Figure 4.10: Single microphone cough sound acquisition $\left(D_{\text {mik-10-speaker }}=50 \mathrm{~cm}\right)$.

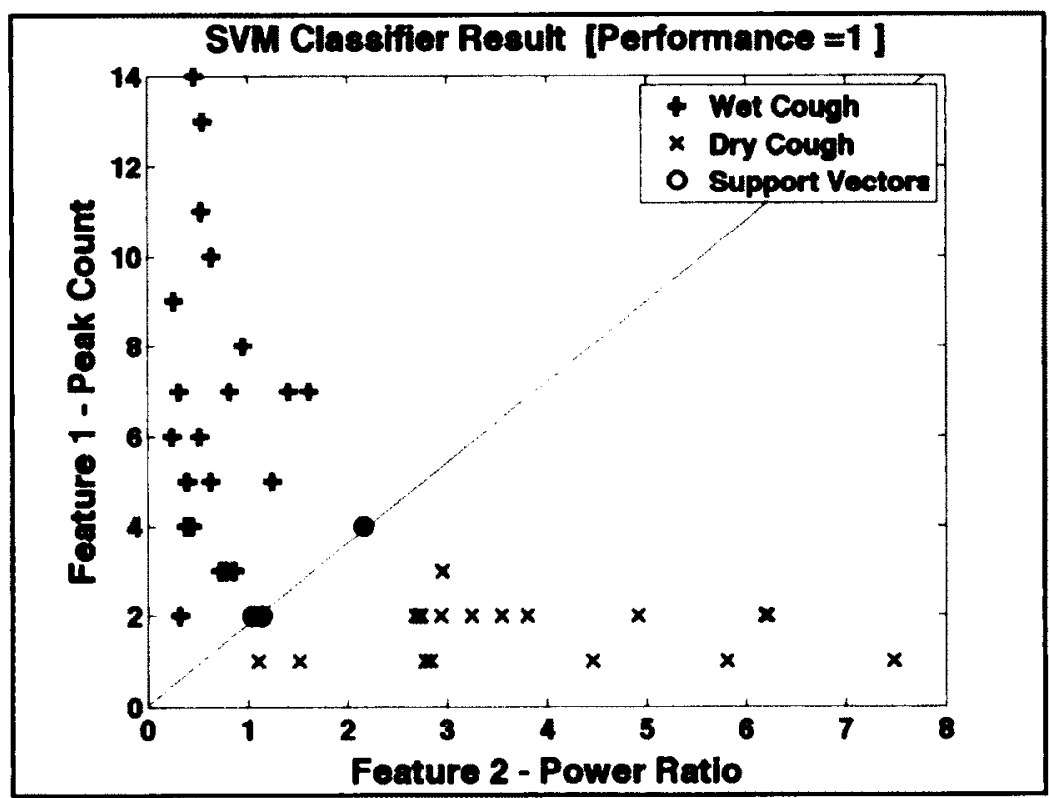

Figure 4.11: Single microphone cough sound acquisition $\left(D_{\text {mic-10-speaker }}=100 \mathrm{~cm}\right)$. 


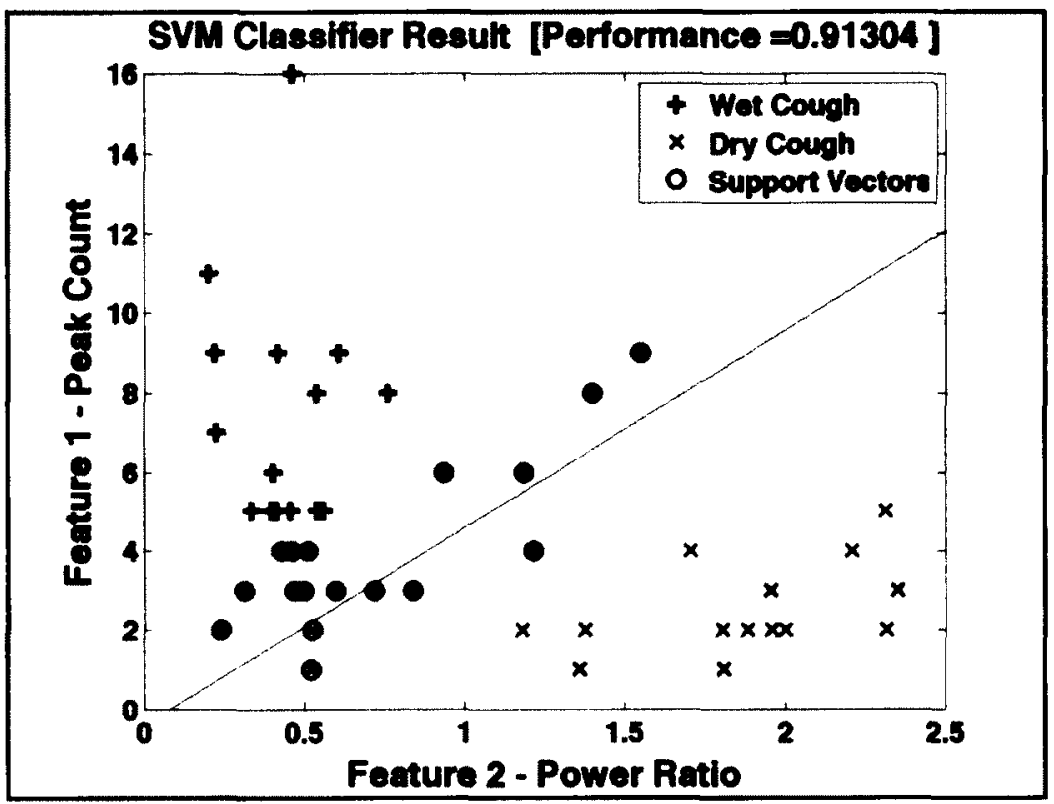

Figure 4.12: Single microphone cough sound acquisition $\left(D_{\text {mic-to-speaker }}=150 \mathrm{~cm}\right)$.

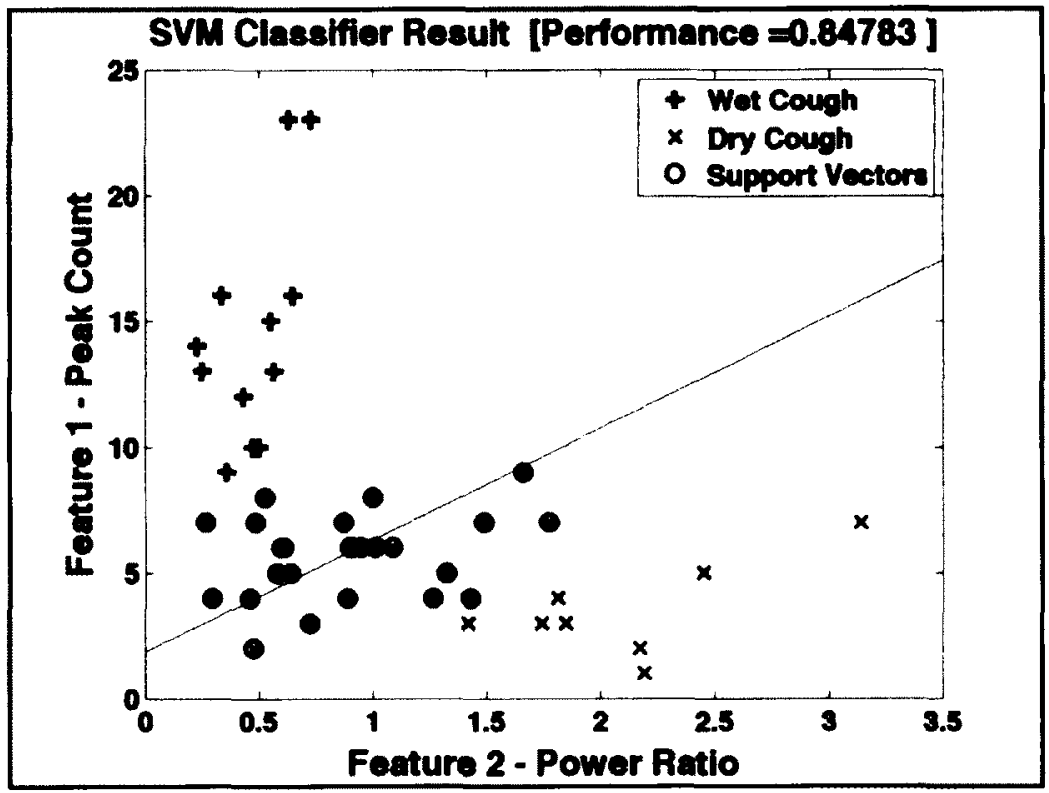

Figure 4.13: Single microphone cough sound acquisition $\left(D_{\text {mic-to-spenker }}=200 \mathrm{~cm}\right)$. 


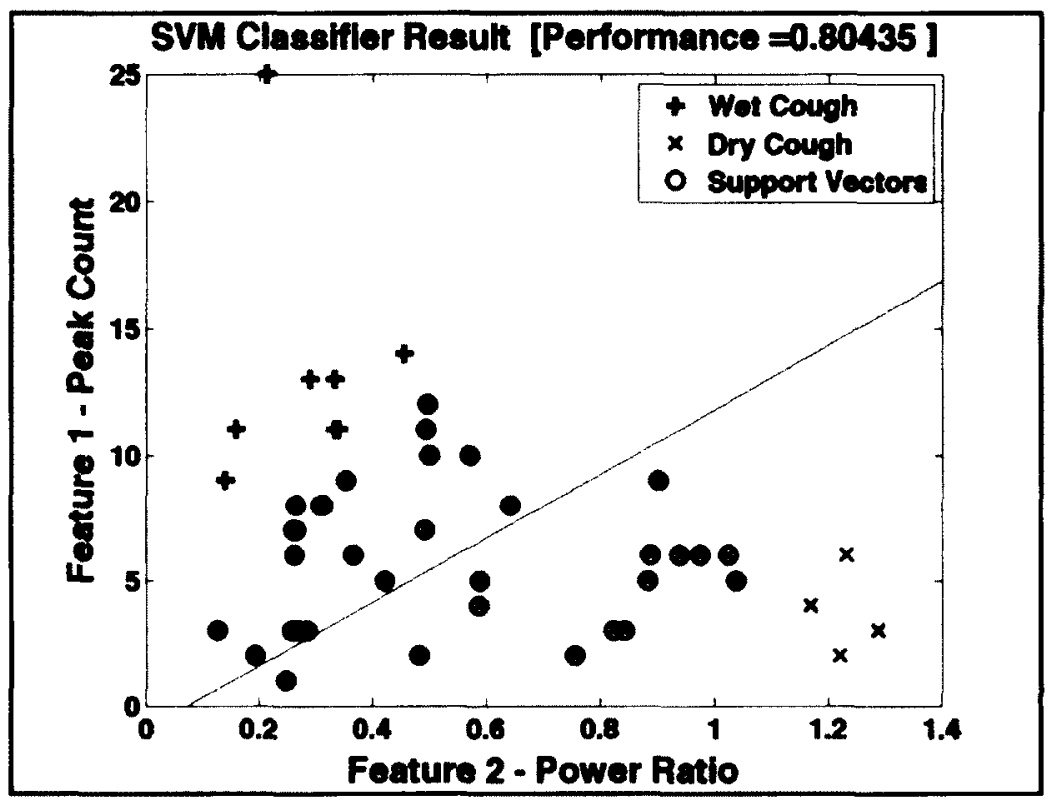

Figure 4.14: Single microphone cough sound acquisition $\left(D_{\text {mic-to-speaker }}=250 \mathrm{~cm}\right)$.

Table 2: Single microphone cough sound acquisition Linear Separation Score

\begin{tabular}{|c|c|}
\hline 50 & 1 \\
\hline 50 & 1 \\
\hline 100 & 0.91 \\
\hline 150 & 0.84 \\
\hline 200 & 0.80 \\
\hline 250 & \\
\hline
\end{tabular}

In this section, experiments were performed to study the effect of room reverberation and microphone-to-speaker distance on the performance of the cough sound discriminator. In the next section, effect of noise on the cough sound discriminator is studied. 


\subsection{Effect of White Noise}

Reverberation is not the only source of cough sound distortion in a typical cough sound acquisition environment. Various types of noise could also distort the captured cough sounds and hence decrease the performance of the cough sound discriminator.

In order to study the effect of noise on the performance of the cough sound discriminator, white noise was played through a second speaker while the cough sound acquisition was being performed. Figure 4.15 depicts the experimental setup used in this section of this thesis.

The white noise was placed $100 \mathrm{~cm}$ at an angle of roughly $60^{\circ}$ from the center of the microphone array structure. The volume of the white noise was tuned to be roughly equal to the loudest cough sound volume and played at $-15 \mathrm{~dB}$ using Adobe Audition. 


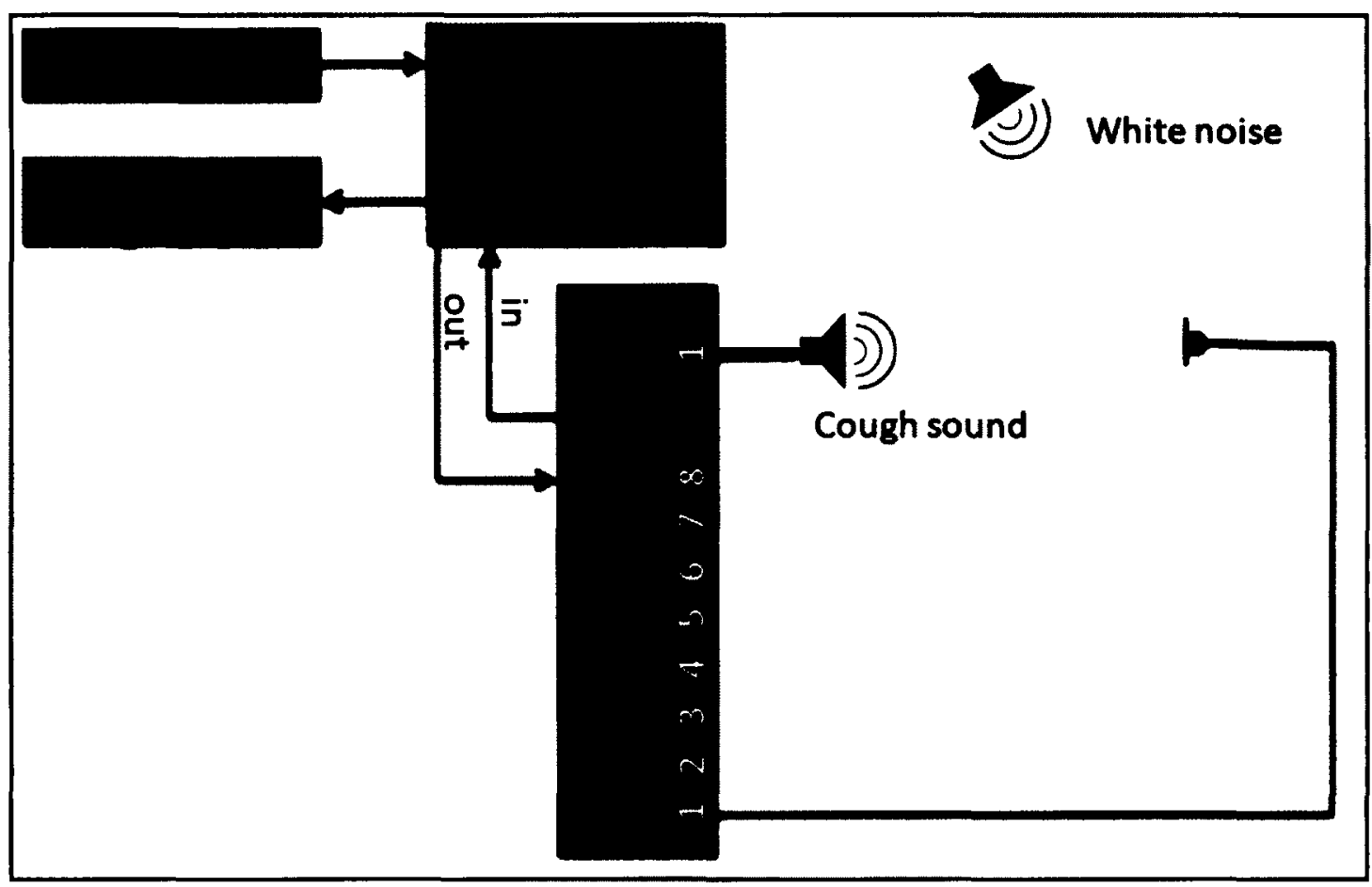

Figure 4.15: Single microphone cough sound acquisition with added white noise.

This section of the thesis is divided into two sets of experiments. The first experiment investigates the effect of white noise on the performance of the cough sound discriminator at different microphone-to-speaker distances. The second experiment investigates the effect of white noise on the performance of the cough sound discriminator at different volume levels of the played cough sounds.

\subsubsection{Microphone to Speaker Distance}

A total of two experiments were performed where the distance between the speaker and microphone was varied while keeping the volume and distance of the white noise emitter 
constant. The experiments were performed at microphone-to-speaker distances of $50 \mathrm{~cm}$, and $200 \mathrm{~cm}$.

Figure 4.16 and Figure 4.17 depict the result of cough sound acquisition with a single microphone and added white noise. As it could be seen, the performance of the cough sound discriminator decreases significantly when white noise is added to the system. Furthermore, at the distance of $200 \mathrm{~cm}$, the discriminator fails to discriminate between the two cough types. Table 3 summarizes these results and compares them with the results obtained previously.

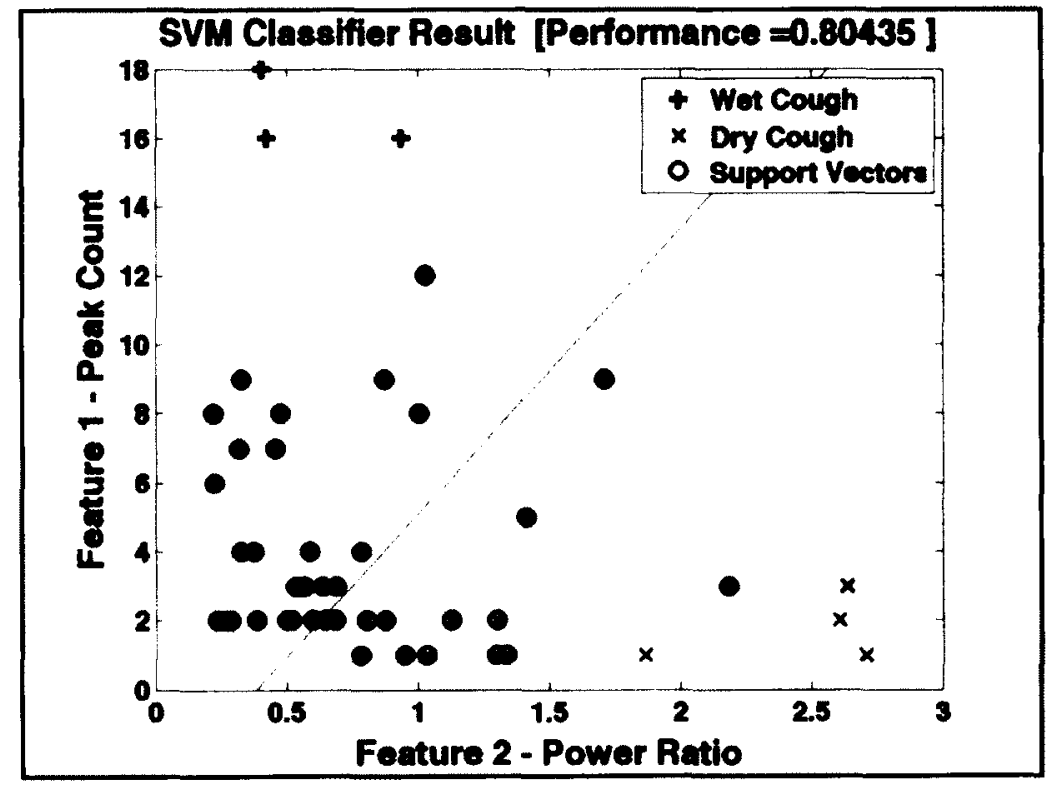

Figure 4.16: LSS of single-microphone and white noise $\left(D_{\text {mik-10-spenker }}=50 \mathrm{~cm}\right)$. 


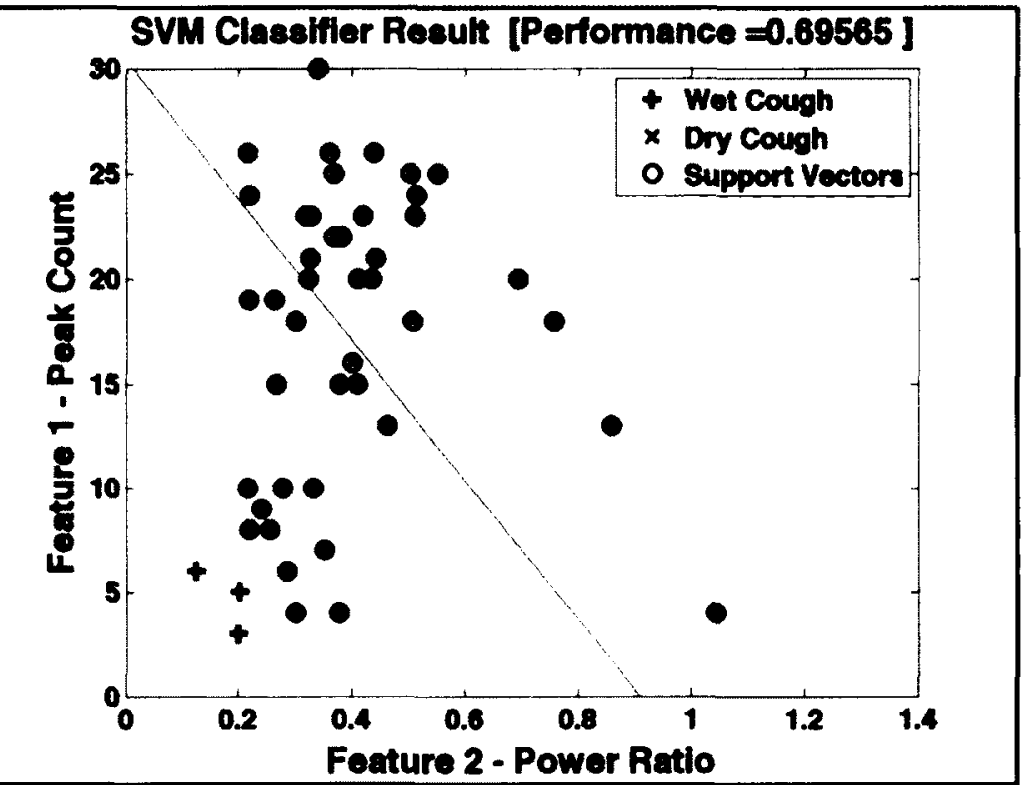

Figure 4.17: LSS of single-microphone and white noise $\left(D_{\text {mk-10-spenker }}=200 \mathrm{~cm}\right)$.

Table 3: LSS of single microphone cough sound acquisition with and without white noise

\begin{tabular}{|c|c|c|}
\hline 5 & 1 & 1 \\
\hline 50 & 1 & 0.80 \\
\hline 200 & 0.84 & 0.69 \\
\hline
\end{tabular}

\subsubsection{Cough Sound Volume Level}

In order to analyze the relationship between the injected white noise and the volume of the played cough sounds, the described experiment was repeated with different cough sound volume levels.

The experimental setup in this section was similar to that depicted in Figure 4.15. The volume level of the played cough sounds was modified using Adobe Audition software. The distance between the speaker and the microphone was set to $200 \mathrm{~cm}$. 
Figure 4.18 and Table 4 summarize the results obtained in this section of the thesis. As it could be observed, the performance improves as the volume of the cough sounds increase. However, the performance decreases at very high volume levels due to system saturation.

Table 4: LSS of single microphone cough sound acquisition with white noise and variable cough sound volume

\begin{tabular}{|c|c|c|c|c|c|c|c|}
\hline & \multicolumn{7}{|c|}{ 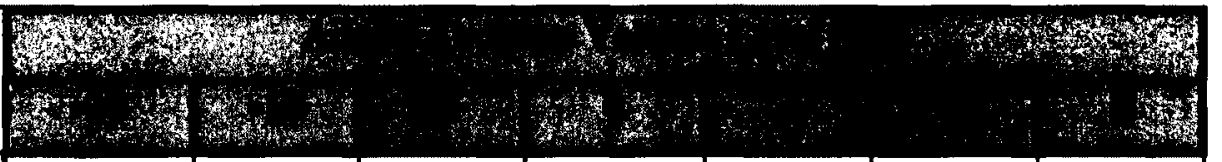 } \\
\hline 18 & 0.61 & 0.69 & 0.69 & 0.71 & 0.71 & 0.73 & 0.69 \\
\hline
\end{tabular}

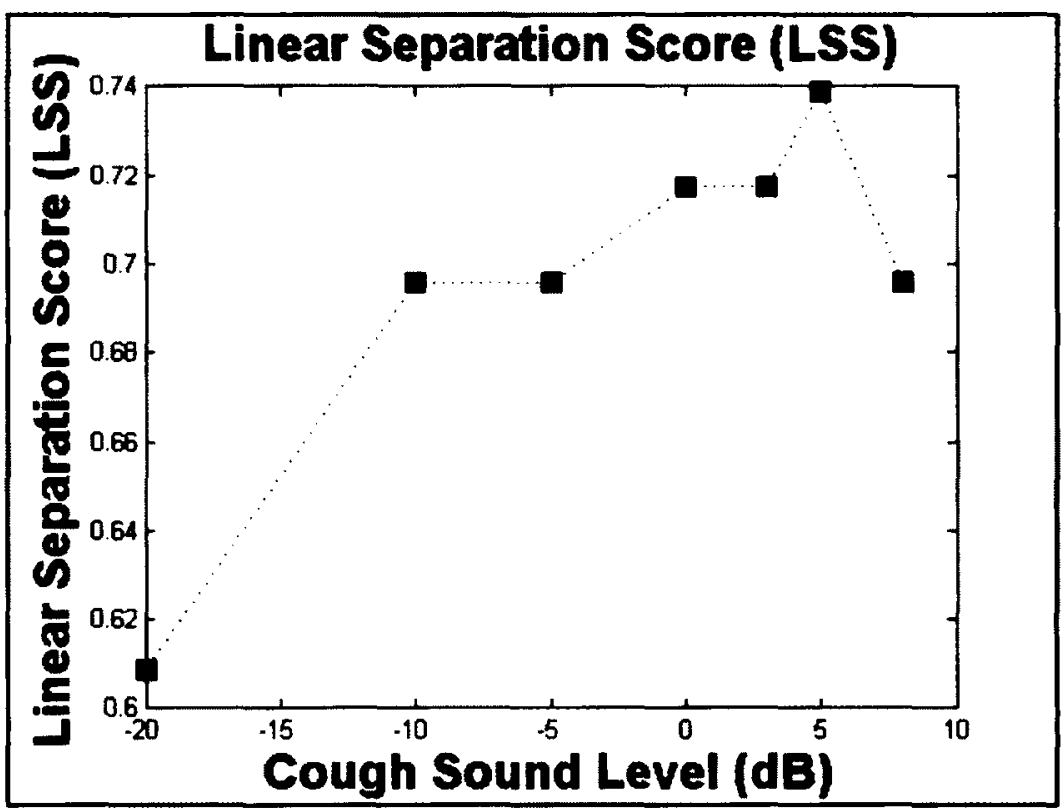

Figure 4.18: LSS of variable cough volume with single microphone. 


\subsection{Discussion}

In this chapter of the thesis, the performance of the cough sound discriminator was investigated in reverberant and noisy environments using only one microphone as the primary audio capture device.

The first experiment involved playing cough sounds from a speaker and capturing it from a microphone from different distances. The distances ranged from $50 \mathrm{~cm}$ to $250 \mathrm{~cm}$. From the results summarized in Table 2 , it could be concluded that as the distance between the speaker and microphone increases, the performance of the cough sound discriminator decreases. The cough sound discriminator was able to distinguish between dry and wet cough sounds successfully and achieve an LSS score of 1 for distances of $50 \mathrm{~cm}$ and $100 \mathrm{~cm}$. The LSS score, however, decreased as the distance increased beyond $100 \mathrm{~cm}$. The decline in performance is because as the distance between the speaker and microphone increases, the amplitude of the captured cough sounds decreases and therefore the signal-to-noise ratio decreases. The decrease in the signal-to-noise ratio causes the dry cough sounds to appear more like wet coughs sounds. The reason why dry coughs start looking more like wet coughs is related to the difference between the second phase of a dry and wet cough sound. As discussed previously, one of the distinguishing factors considered in the cough sound discriminator algorithm was the number of peaks of the energy envelope of the second phase of the cough sound [16]. As the signal-to- 
noise ratio decreases in a cough signal, the second phase of the cough signal start having an increase in spikes. This increase in spikes makes a dry cough look more like a wet cough and as a result the performance of the cough sound discriminator decreases. The similarity between dry and wet cough sounds refer to the similarity in the outcome of the cough sound discriminator (i.e. same number of peaks) and not the actual cough signal.

The second experiment involved adding white noise to the experimental setup while playing cough sounds from various distances to the single microphone. Two distances were selected for this part of the setup: $50 \mathrm{~cm}$ and $200 \mathrm{~cm}$. The $50 \mathrm{~cm}$ distance resulted in an LSS of 1 in the absence of white noise. When white noise was added, however, the performance decreased to 0.80 . The location, distance and volume of the white noise source were selected arbitrarily. Changing any of the mentioned factors would have obviously resulted in a different LSS score. Nevertheless, the experiment showed that white noise decreases the performance of the cough sound discriminator. Keeping the distance between the white noise source and microphone constant and increasing the distance between the cough sound source and microphone, significantly decreased the signal-to-noise ratio and as a result, decreased the LSS. In fact, for the $200 \mathrm{~cm}$ distance, the cough sound discriminator failed to properly distinguish between the two types of cough sounds. From Figure 4.17, it can be seen that the dry and wet cough sounds have similar values for their Feature 2. In addition, the dry cough signals have greater number of peaks than wet cough signals. This is because the added white noise has introduced 
many peaks to the second phase of the dry cough signal, making it appear more like a wet cough signal. The results obtained in this section show that the features extracted by the cough sound discriminator do not perform well in the presence of white noise.

The third experiment investigated the effect of the volume of cough sounds on the performance of the cough sound discriminator in the presence of white noise. This experiment had a similar setup as both the first and second experiment. As it could be seen from Figure 4.18 and Table 4, as the volume of the cough sound increased, the LSS also improved. This is due to the fact that higher volume levels of the cough sound increases the signal-to-noise ratio and hence improves the performance of the discriminator. At very high volume levels however, the performance starts to decrease again. This decrease is because the output port of the MOTU audio interface saturates at high volumes. Furthermore, the speaker's non-linear behaviour increases at higher vibration levels caused by higher volume. Although the decrease in the LSS at high volumes of the cough sound is a physical limitation of the experimental setup, it also represents an actual patient situation. At close distances, the patient might cough at higher volume levels which might cause saturation within the microphone and hence decrease the performance of the cough sound discriminator.

\subsection{Conclusions}

This chapter investigated the performance of the cough sound discriminator in reverberant and noisy environments. Three sets of experiments were performed in this 
section. The first experiment examined the effect of room reverberation and the distance between speaker and microphone. It was concluded from this experiment that the performance of the cough sound discriminator decreases as the distance between the speaker and microphone increases. In the second experiment, white noise was added to the cough sound acquisition experiment in order to investigate the effect of white noise on the discriminator. The second experiment revealed that the added white noise corrupts the cough sounds, which in turn decreases the cough sound discriminator performance. Finally, the effect of cough sound volume in the presence of white noise was investigated in the third experiment. The results from the third experiment revealed that an increase in cough sound volume improves the performance of the cough sound discriminator, except for very high volume levels which cause system saturation and increase in system's nonlinear behaviour.

The experimental results obtained in this section reveal the limitations of the cough sound discriminator in noisy and reverberant environments. The distortion introduced by the noise and reverberation had a significant impact on the performance of the cough sound discriminator. The noise level and the distance between the microphone, desired sound source and the source of noise impacted the cough sound discriminator's ability to distinguish between dry and wet cough sounds. The results suggest that a single microphone would not be able to capture cough signals in noisy and reverberant environments in such a way that it would result in a high Linear Separation Score. As a 
result, the use of microphone arrays as a way of improving the performance of the cough sound discriminator will be investigated in the next chapter. 


\section{Chapter 5:}

\section{Cough Sound Acquisition using Microphone}

\section{Array}

In the previous chapter of this thesis, the performance of the cough sound discriminator was investigated in reverberant and noisy environments using single-microphone cough sound acquisition. The experiments conducted in the previous chapter revealed a decrease in the performance of the cough sound discriminator when a single microphone was used to capture cough sounds.

In this chapter, the use of microphone arrays in improving the performance of the cough sound discriminator is investigated.

The chapter starts with an introduction on the experimental setup followed by a detailed explanation on the various experimentations performed using microphone arrays. 
The results obtained are discussed and analysed in great detail followed by a chapter conclusion.

\subsection{Experimental Setup}

The experimental equipment used in this chapter is similar to that used in section 4.1. The main difference between the two setups is the number of microphones used and their orientation. A maximum of 7 microphones were used in various arrangements throughout this chapter.

The microphones within the microphone array were arranged facing the sound source and perpendicular to the wavefront of the cough sounds. The microphone array was located directly in front of the speaker similar to Figure 5.1. Cough sounds were played through the speaker via the output of the MOTU audio interface and captured through the analog input of the MOTU device. The recorded cough sounds were passed through the delay-and-sum beamforming algorithm. The output of the beamformer was finally passed through the cough sound discriminator system and the performance of the discriminator was determined using the Linear Separation Score. The volume level at the output of the Adobe Audition software was set to $-10 \mathrm{~dB}$. This level was chosen since it did not cause output saturation in any of the played cough sounds.

The experimentation performed in this section is similar to that of the previous section, with the difference that multiple microphones were used for the cough sound acquisition as shown in Figure 5.1. Introducing more microphones to the experimental 
setup also introduces more factors that could be considered when capturing sounds using the microphone array. Some of those factors are:

- The distance between the microphones (microphone-to-microphone distance)

- The distance between the microphones and the sound source (microphone-tospeaker distance)

- The number of microphones used for the cough sound acquisition

In order to investigate the effect of each of the mentioned factors on the performance of the cough sound discriminator, experiments were performed taking into account all the possible values for each of the experimental variables. These possible values are summarized in Table 5. The effect of these factors on the performance of the cough sound discriminator will be analysed in this chapter.

Table 5: Microphone array cough sound acquisition experimental variables

\begin{tabular}{|c|c|}
\hline Microphone-to-Microphone Distance & $5 \mathrm{~cm}, 10 \mathrm{~cm}, 15 \mathrm{~cm}, 20 \mathrm{~cm}$ \\
\hline Microphone-to-Speaker Distance & $50 \mathrm{~cm}, 100 \mathrm{~cm}, 150 \mathrm{~cm}, 200 \mathrm{~cm}, 250 \mathrm{~cm}$ \\
\hline Number of Microphones & $1,2,3,5,7$ \\
\hline
\end{tabular}




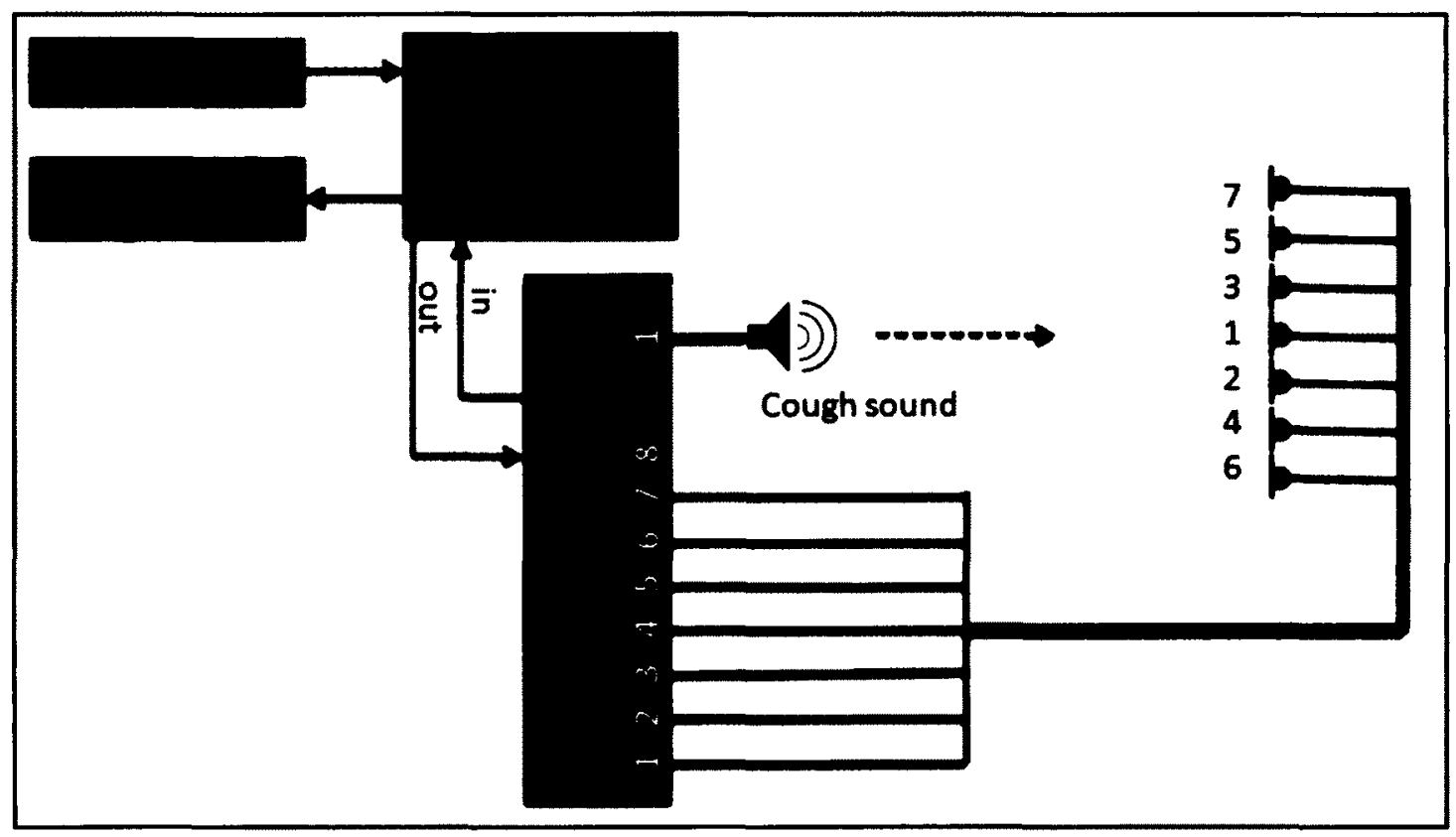

Figure 5.1: Microphone array experimental setup.

\subsection{Delay-and-Sum Beamformer}

The microphone array used in this section of the thesis consisted of 2 to 7 microphones arranged in a linear fashion as described previously. Delay-and-sum (DAS) beamforming algorithm was used in the cough sound acquisition setup. The signals acquired by the microphones were passed through the DAS beamformer and the output of the DAS beamformer was fed into the Linear Separation Score system as depicted in Figure 3.3. Figure 5.2 shows the block diagram of the delay-and-sum beamformer algorithm. 


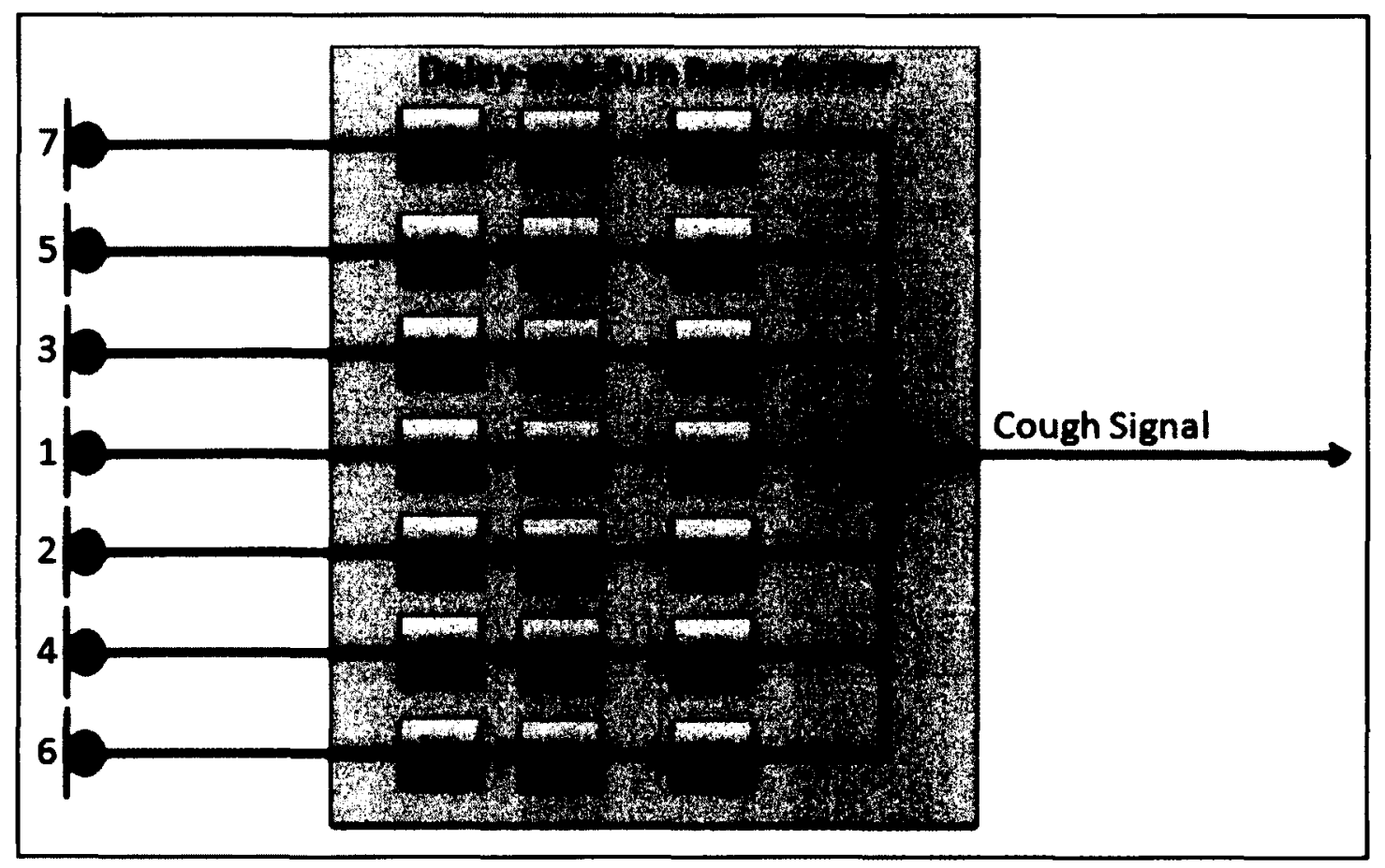

Figure 5.2: Delay-and-sum beamformer.

The most critical component of the delay-and-sum beamformer is the relative timedelay between the microphones [63]. In order to compute this delay, two different techniques were used in order to confirm the validity of this critical component.

The first method, used mathematics and geometry in order to compute the relative delay between two microphones in units of samples points. The delay between the samples of adjacent microphones could be calculated from Figure 5.3 as shown in (7): 


$$
\text { delay }_{\text {Number of Samples }}=\left[\frac{D \sin \left[\tan ^{-1}\left(\frac{D}{x}\right)\right]}{v}\right] \cdot F_{s}
$$

where $v$ is the speed of sound, $F_{s}$ is the sampling rate $(44.1 \mathrm{kHz}), D$ is the distance between adjacent microphones and $x$ is the distance between speaker and the middle microphone (microphone \#1).

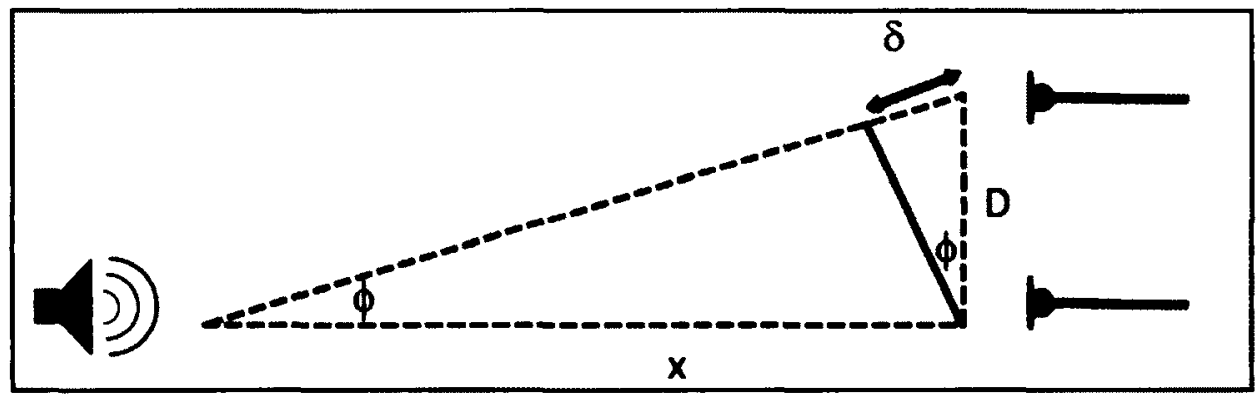

Figure 5.3: Mathematical method for cakulating delay,

The second method calculated the delay between adjacent microphones using an experimental approach. In this method, a sine wave of frequency $2 \mathrm{kHz}$ was played from the speaker and captured by all the microphones within the microphone array at $44.1 \mathrm{kHz}$ sampling rate. The first peak of the sine wave captured by the microphones was analysed and the relative delay was determined using the offset of the first peak. Figure 5.4 depicts a sample output of the sine waves captured by the microphones in the microphone array. 
As it could be seen from the figure, microphone \#1 is the first microphone to capture the sine wave (dark blue wave), followed by microphones \#2 and \#3 (orange waves), microphones \#4 and \#5 (green waves) and microphones \#6 and \#7 (pink waves). The microphones were numbered in the experimental setup shown in Figure 5.1. The vertical lines demonstrate the delay between the first peaks of the sine waves. The color of the vertical line matches the color of the sine waves.

The delays obtained from the two methods were compared and in all cases, the results were very close with one another. In case of major differences, the experimental method was repeated in order to further confirm the calculated delay. The computed results were then fed to the delay-and-sum beamforming algorithm and the final cough sound produced by the algorithm was fed to the cough sound discriminator system and the Linear Separation Score (LSS) was calculated accordingly.

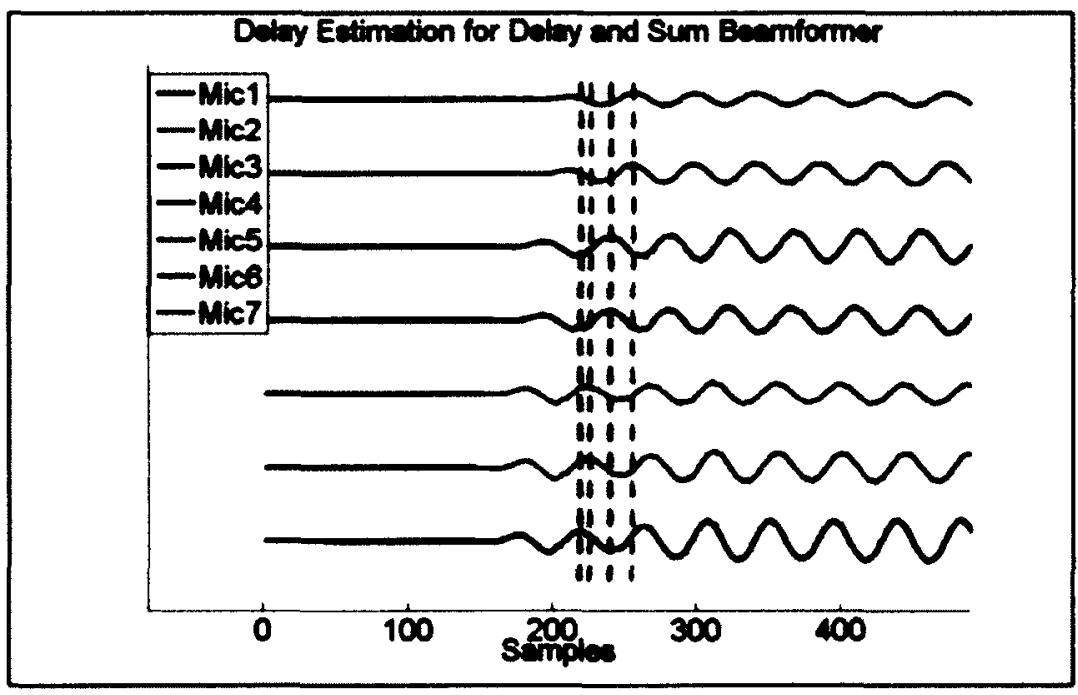

Figure 5.4: Experimental method for calculating delay. 


\subsection{Effect of Reverberation}

In section 4.3 , the effect of microphone-to-speaker distance was studied on the performance of the cough sound discriminator. Results from that section showed that the performance of the cough sound discriminator decreases as the microphone-to-speaker distance increases. In this section, microphone array is used in order to improve the performance of the cough sound discriminator in reverberant environments.

The experimental setup involved in this section explores various configurations of the microphone array in order to obtain a complete understanding of the effect of microphone array on the performance of the cough sound discriminator.

The experiments involved incrementally adding more microphones at a given microphone-to-speaker distance. The microphone-to-speaker distances used in this section were $150 \mathrm{~cm}, 200 \mathrm{~cm}$ and $250 \mathrm{~cm}$. The distance between the microphones being added to the setup was also varied in order to study the effect of microphone-tomicrophone distance on the shape of the beam formed by the microphone array and ultimately on the performance of the cough sound discriminator. The microphone-tomicrophone distances used were $5 \mathrm{~cm}, 10 \mathrm{~cm}, 15 \mathrm{~cm}$ and $20 \mathrm{~cm}$. Finally delay-and-sum beamforming technique was performed on the signals acquired by the microphones within the microphone array. 
Table 6, Table 7 and Table 8 summarize the Linear Separation Score obtained in this experimental setup. Figure 5.5, Figure 5.6 and Figure 5.7 graphically depict the Linear Separation Score obtained using microphone arrays in cough sound acquisition.

Table 6: LSS for microphone array cough sound acquisition $\left(D_{\text {Mic-10-Speaker }}=150 \mathrm{~cm}\right)$

\begin{tabular}{|c|c|c|c|c|}
\hline $\begin{array}{l}3 \\
3 \\
3\end{array}$ & 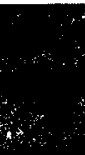 & 3 & & \\
\hline$n^{2}+y^{3}$ & 91 & 91 & 91 & 91 \\
\hline 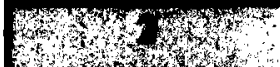 & 91 & 93 & 91 & 91 \\
\hline 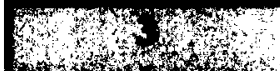 & 95 & 93 & 95 & 93 \\
\hline 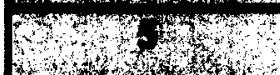 & 95 & 95 & 95 & 91 \\
\hline 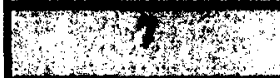 & 95 & 95 & 97 & 93 \\
\hline
\end{tabular}

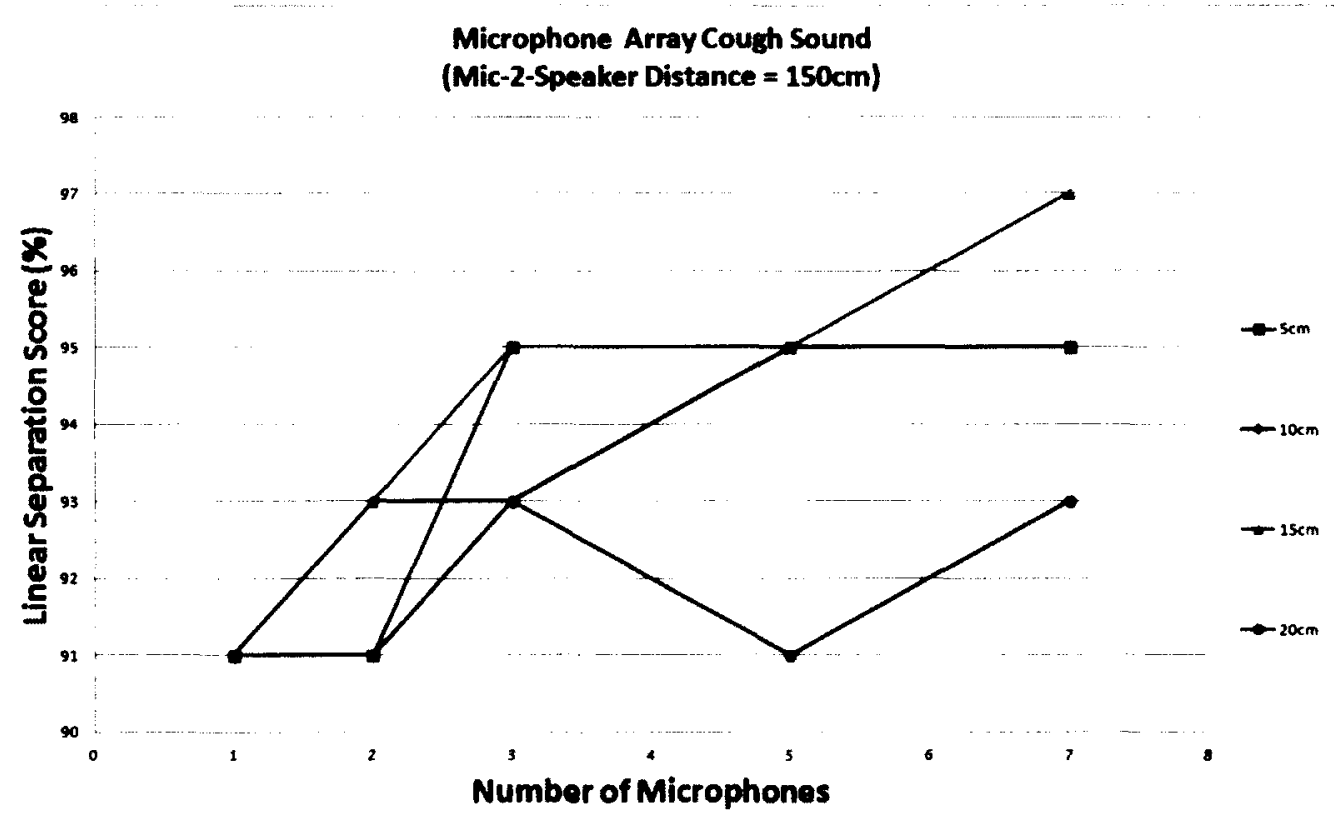

Figure 5.5: Microphone array cough sound acquisition $\left(D_{\text {Mic-2-Speaker }}=150 \mathrm{~cm}\right)$. 
Table 7: LSS for microphone array cough sound acquisition $\left(D_{\text {Mic-to-Speaker }}=200 \mathrm{~cm}\right.$ )

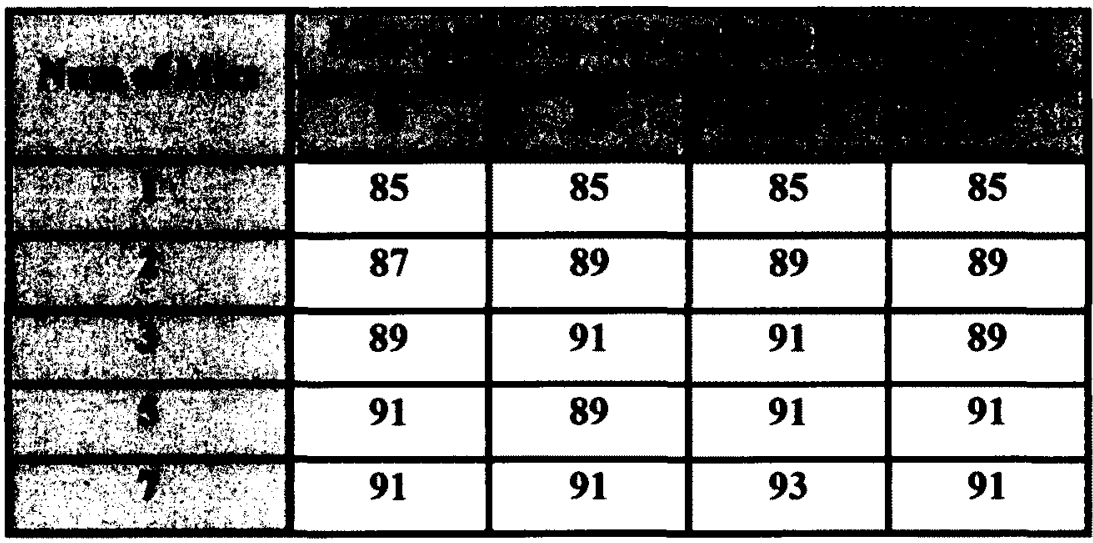

Microphone Array Cough Sound

(Mic-2-Speaker Distance $=200 \mathrm{~cm}$ )

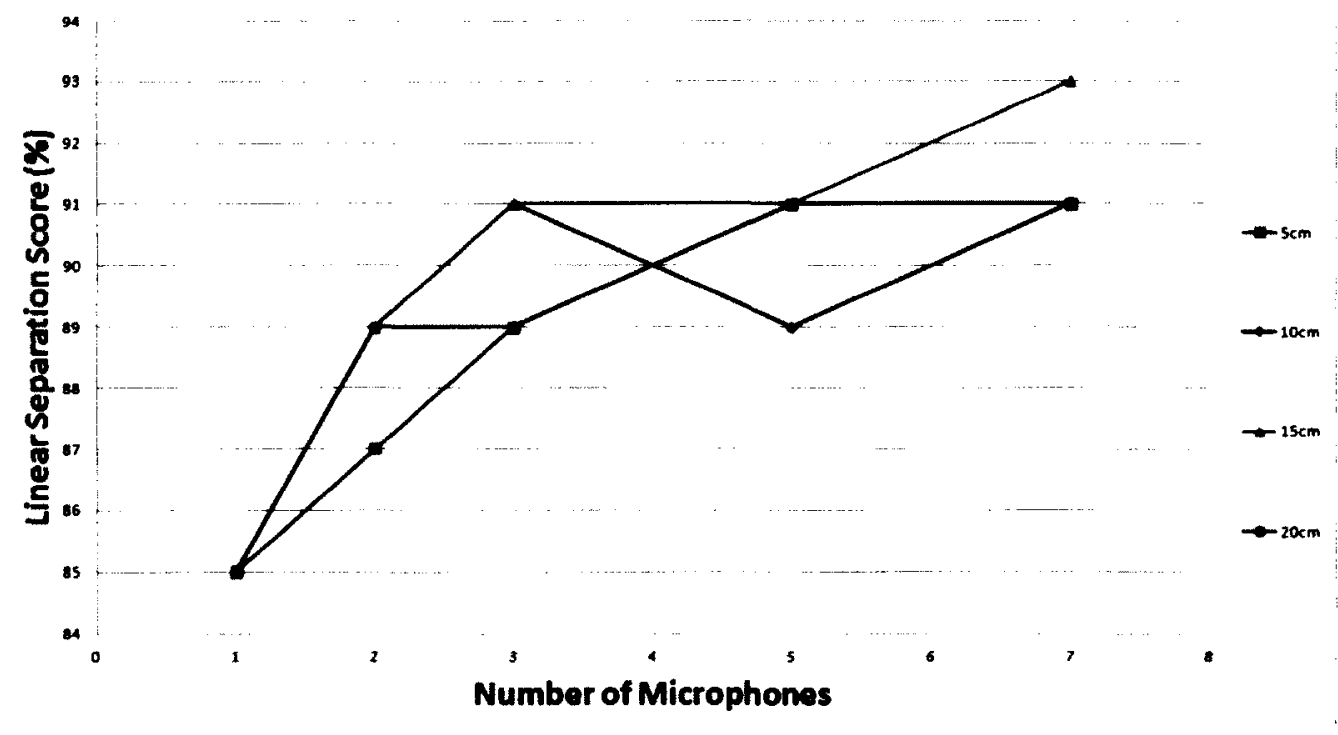

Figure 5.6: Microphone array cough sound acquisition $\left(D_{\text {Mic-2-Speaker }}=200 \mathrm{~cm}\right)$. 
Table 8: LSS for microphone array cough sound acquisition $\left(D_{\text {Mik-10-Speaker }}=250 \mathrm{~cm}\right)$

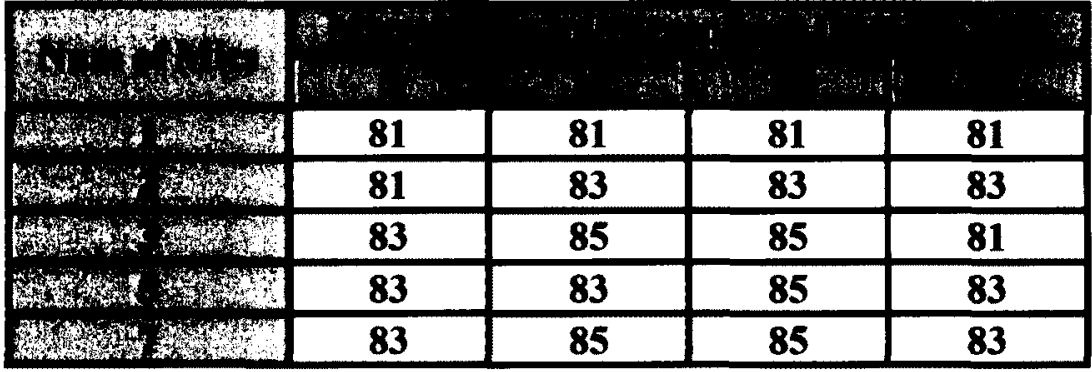

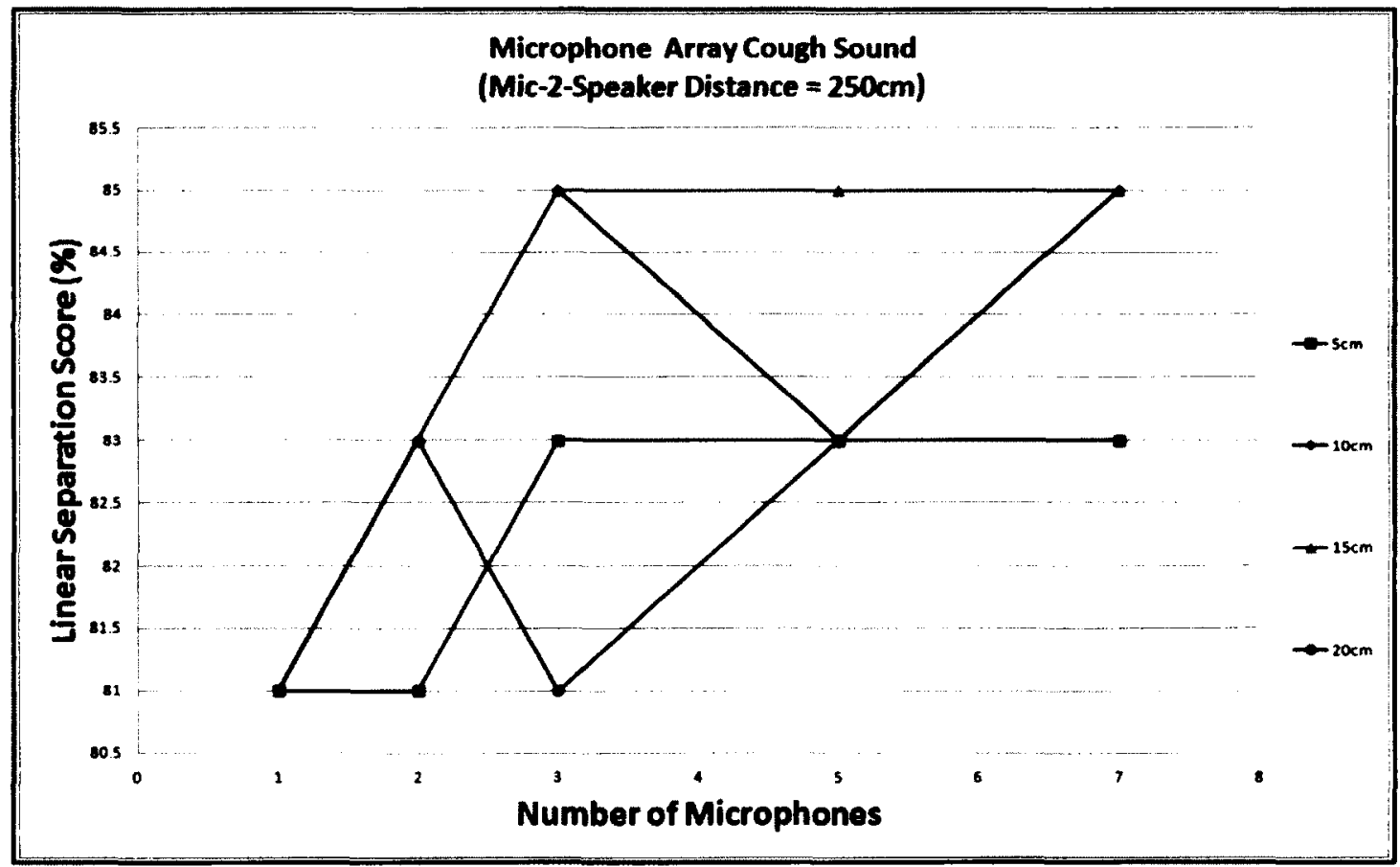

Figure 5.7: Microphone array cough sound acquisition $\left(D_{\text {Mic-2-speaker }}=250 \mathrm{~cm}\right)$. 
Table 9, Table 10, Table 11 summarize the LSS obtained by combining the signals acquired by the other microphones with the microphone array.

Table 9: Complete microphone array LSS $\left(D_{\text {Mk-to-Speaker }}=150 \mathrm{~cm}\right)$

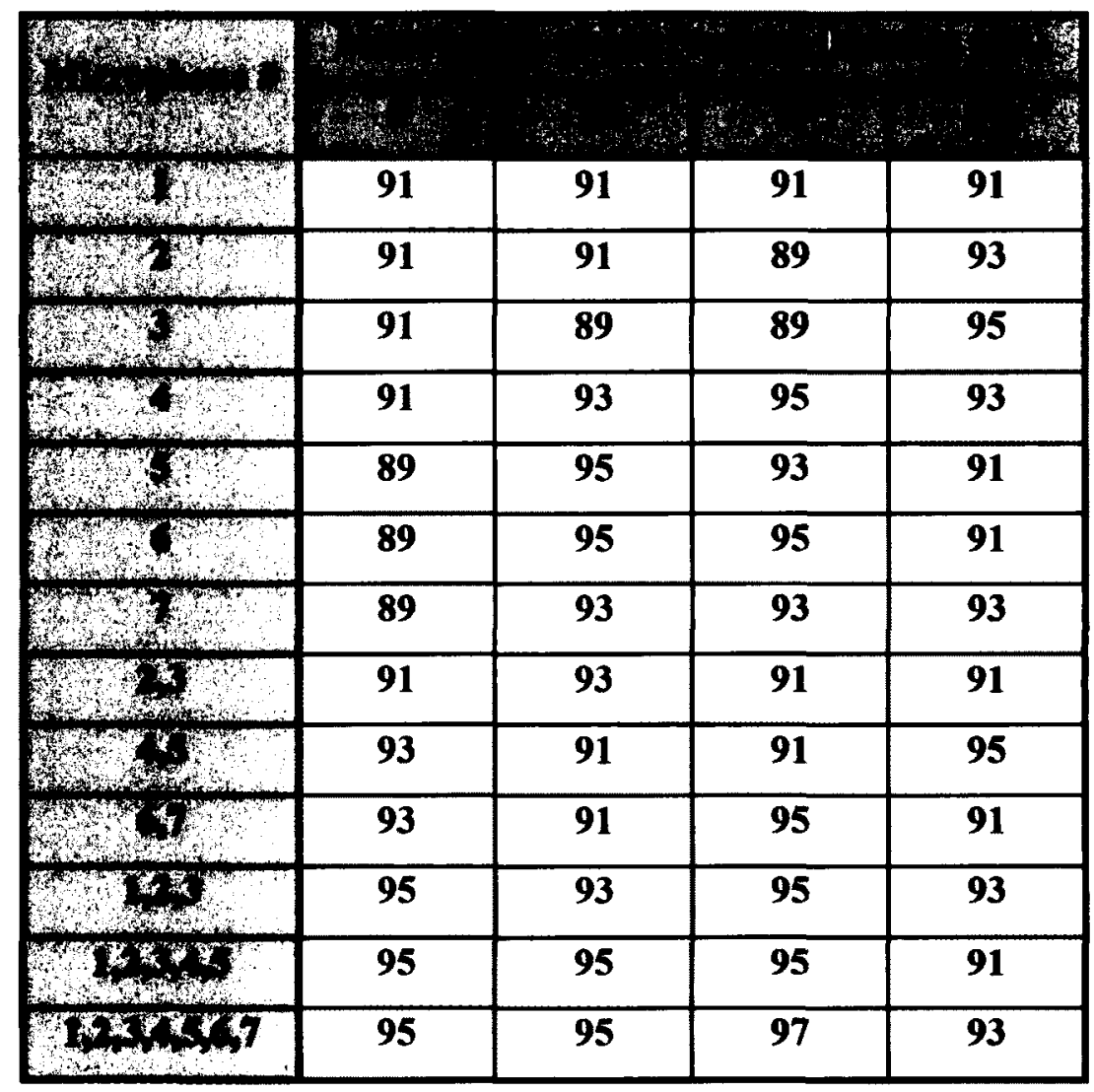


Table 10: Complete microphone array $L S S\left(D_{\text {Mik-1a-Speaker }}=200 \mathrm{~cm}\right)$

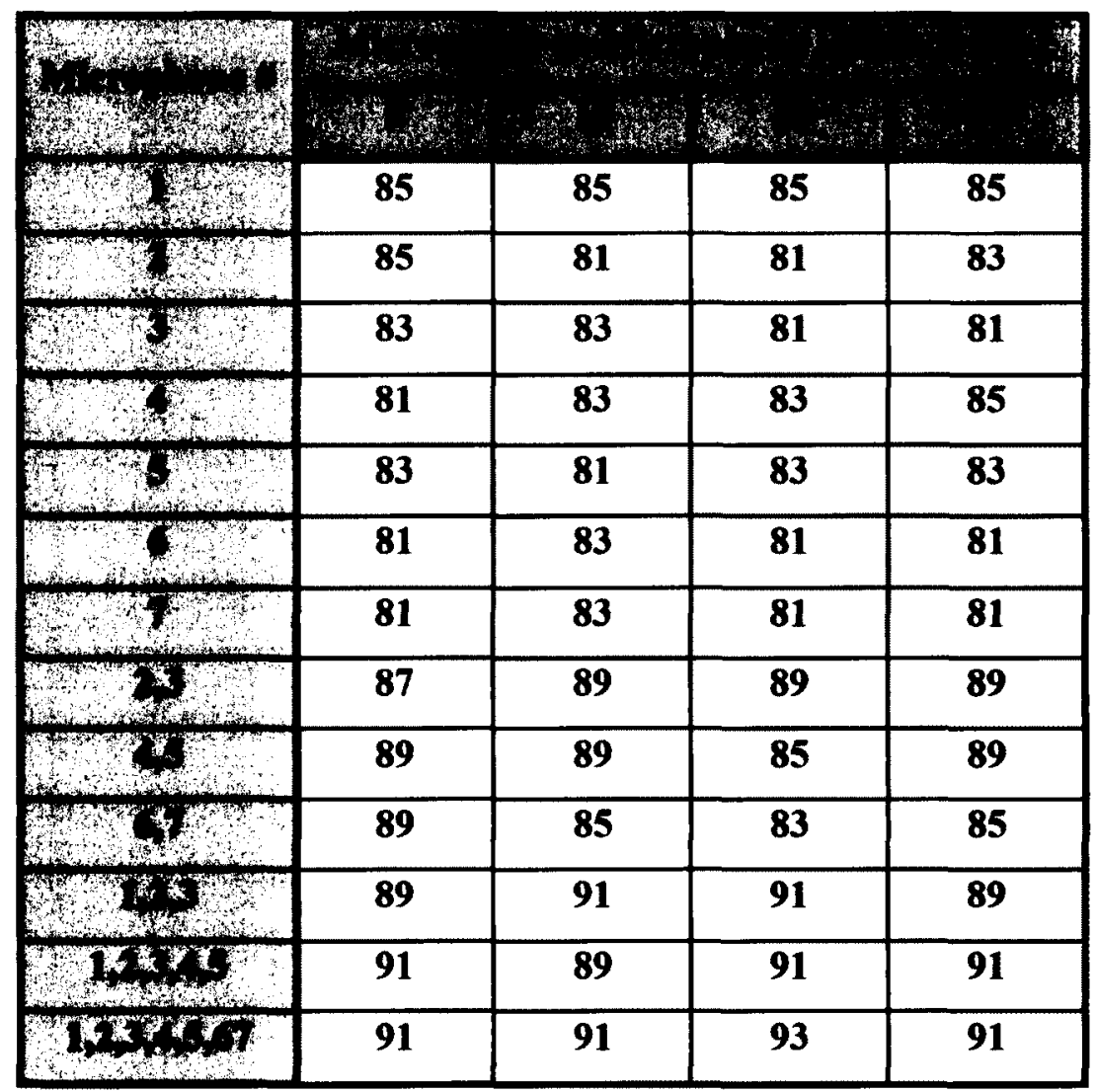


Table 11: Complete microphone array LSS $\left(D_{\text {Mik-to-Spenker }}=250 \mathrm{~cm}\right)$

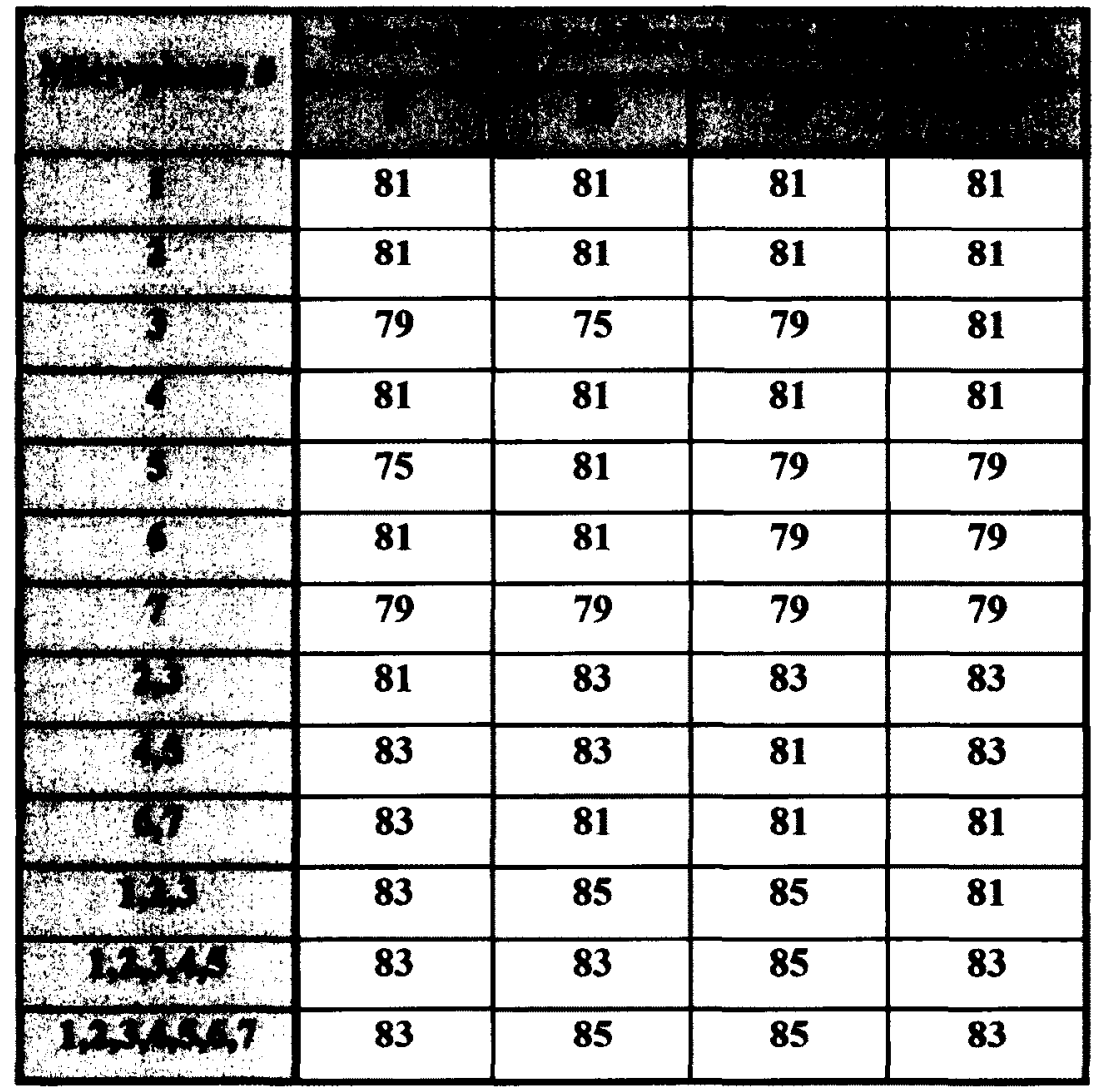

In this section, the performance of the cough sound discriminator was analysed using microphone arrays and delay-and-sum beamforming technique. In the following section, the effect of white noise on the performance of the cough sound discriminator will be analysed. 


\subsection{Effect of White Noise}

In Section 4.4, the effect of white noise on the performance of the cough sound discriminator was analysed using a single microphone for cough sound acquisition. The results summarized in Table 3 indicated that the cough sound discriminator is not capable of distinguishing between dry and wet cough sounds in the presence of white noise. The decrease in the performance of the discriminator was more evident as the distance between the speaker and microphone increased.

In this section, microphone arrays are used in the cough sound acquisition stage of the experiment in order to improve the Linear Separation Score of the cough sound discriminator in the presence of white noise. Figure 5.8 depicts the experimental setup used in this section of the thesis. 


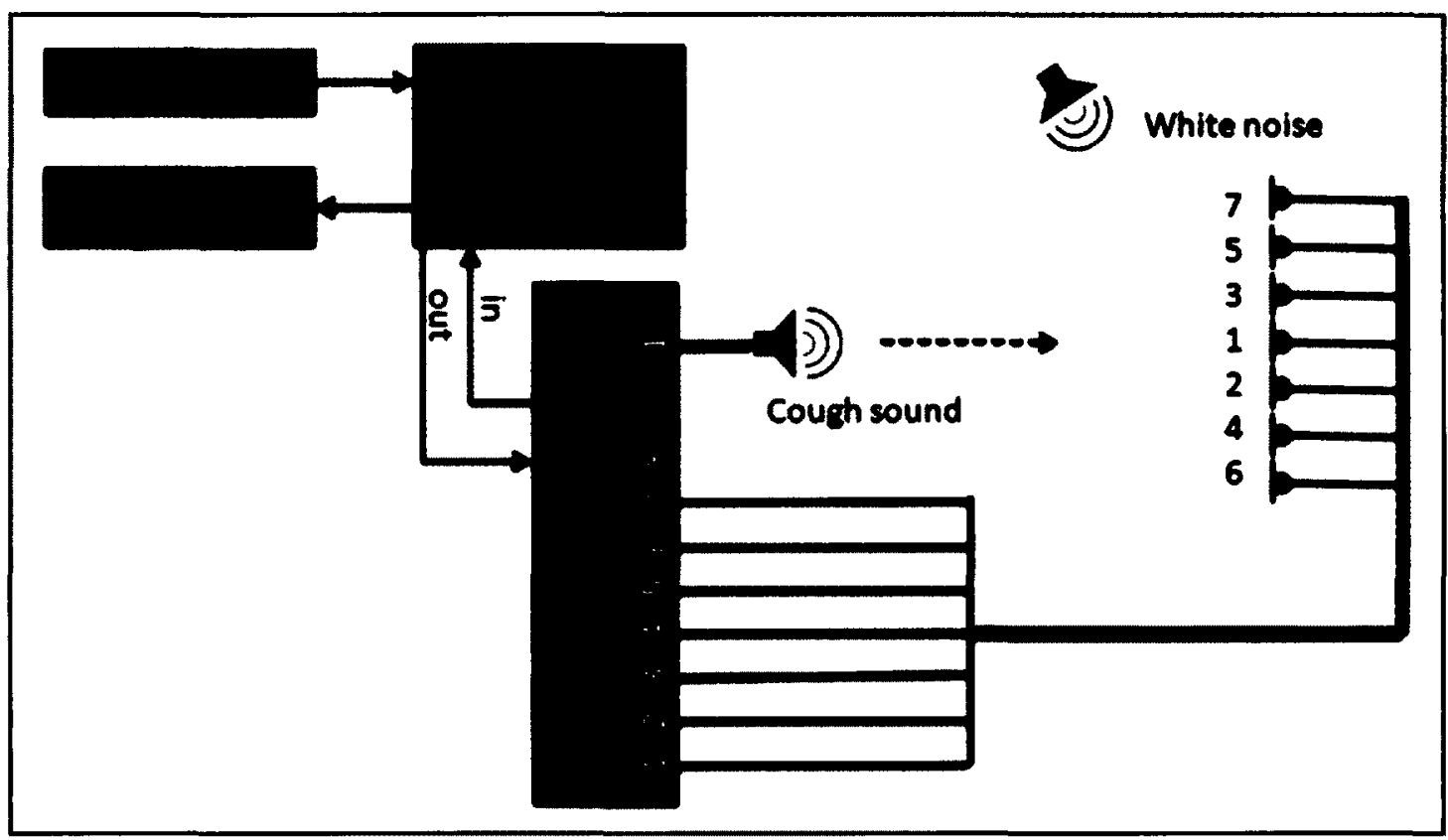

Figure 5.8: Microphone array plus white noise experimental setup.

At it could be seen from the figure, a single white noise source is located near the microphone array structure. The distance of the speaker to microphone array structure is varied while keeping the distance between the white noise source and microphone array constant. Similar to section 4.4.1, microphone-to-speaker distances of $50 \mathrm{~cm}$ and $200 \mathrm{~cm}$ were used.

Table 12 and Table 13 summarize the Linear Separation Score obtained when using microphone arrays in the presence of white noise. Similarly Figure 5.9 and Figure 5.10 graphically depict the results obtained in this section. As it could be seen from the tables and figures, adding more microphones significantly improved the Linear Separation Score of the cough sound discriminator. 
Table 12: Microphone array LSS with white noise $\left(D_{\text {Mic-to-Speaker }}=50 \mathrm{~cm}\right)$

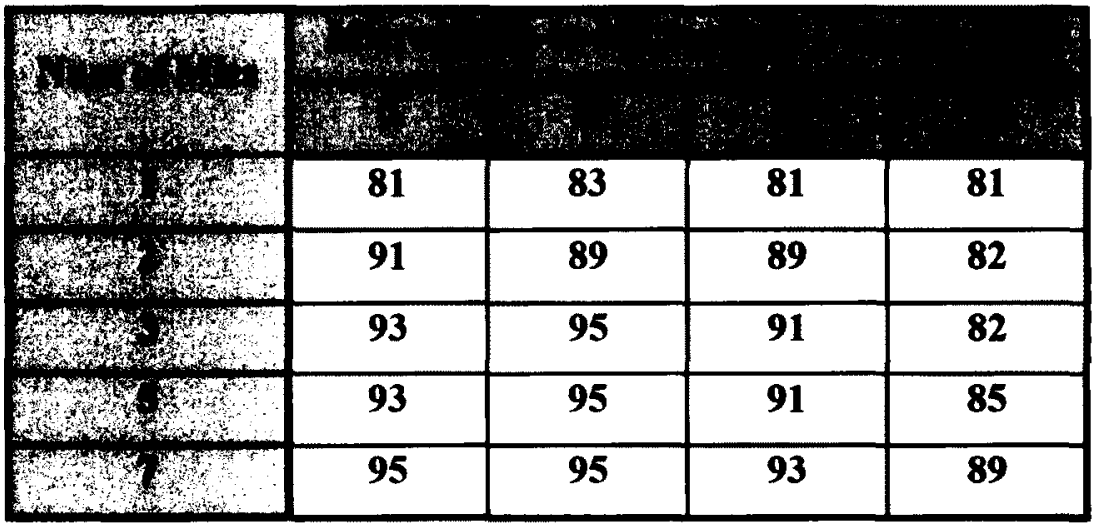

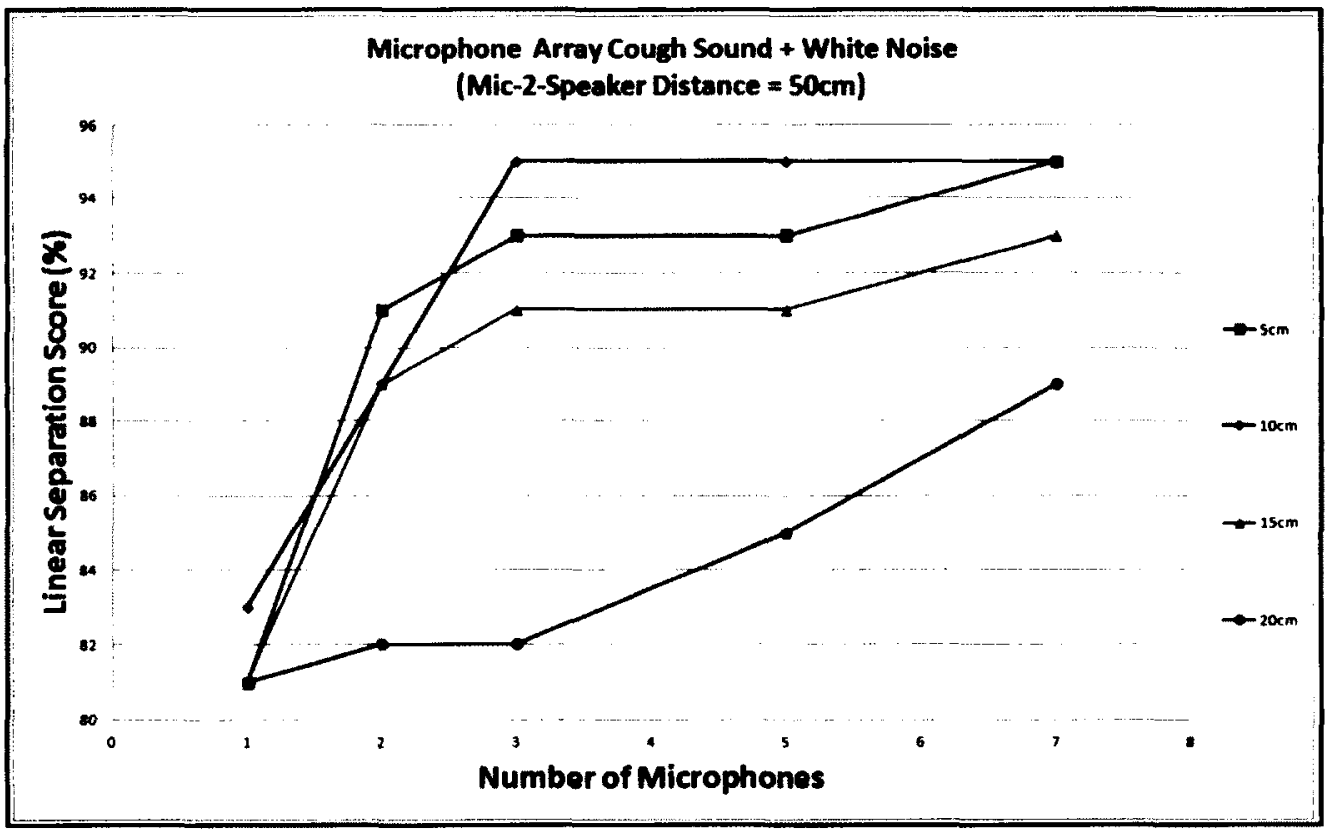

Figure 5.9: Microphone array cough sound acquisition and white noise $\left(D_{M k-2-S p e a k e r}=50 \mathrm{~cm}\right)$. 
Table 13: Microphone array LSS with white Noise $\left(D_{\text {Mik-10-Speaker }}=200 \mathrm{~cm}\right)$

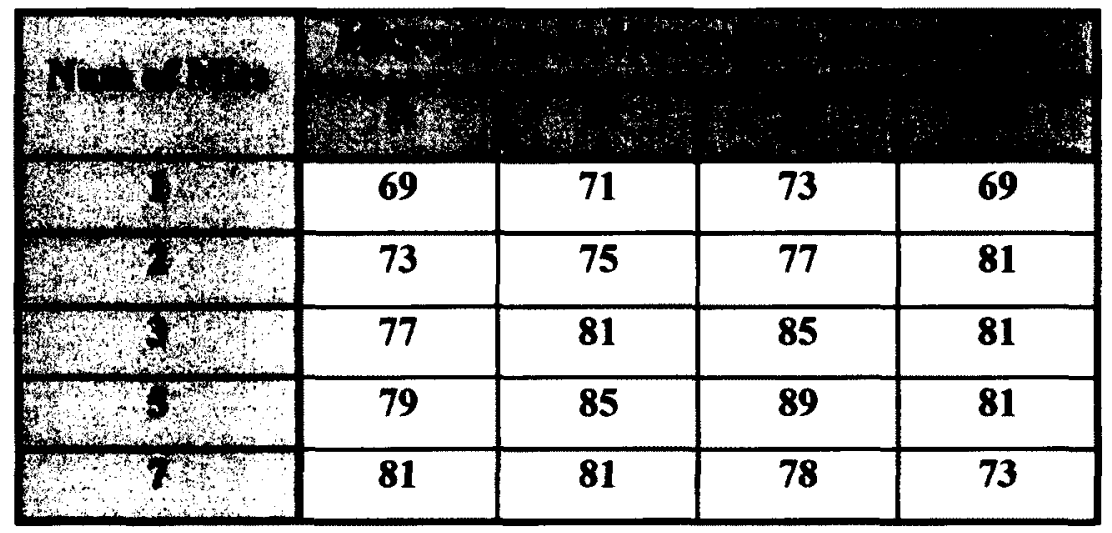

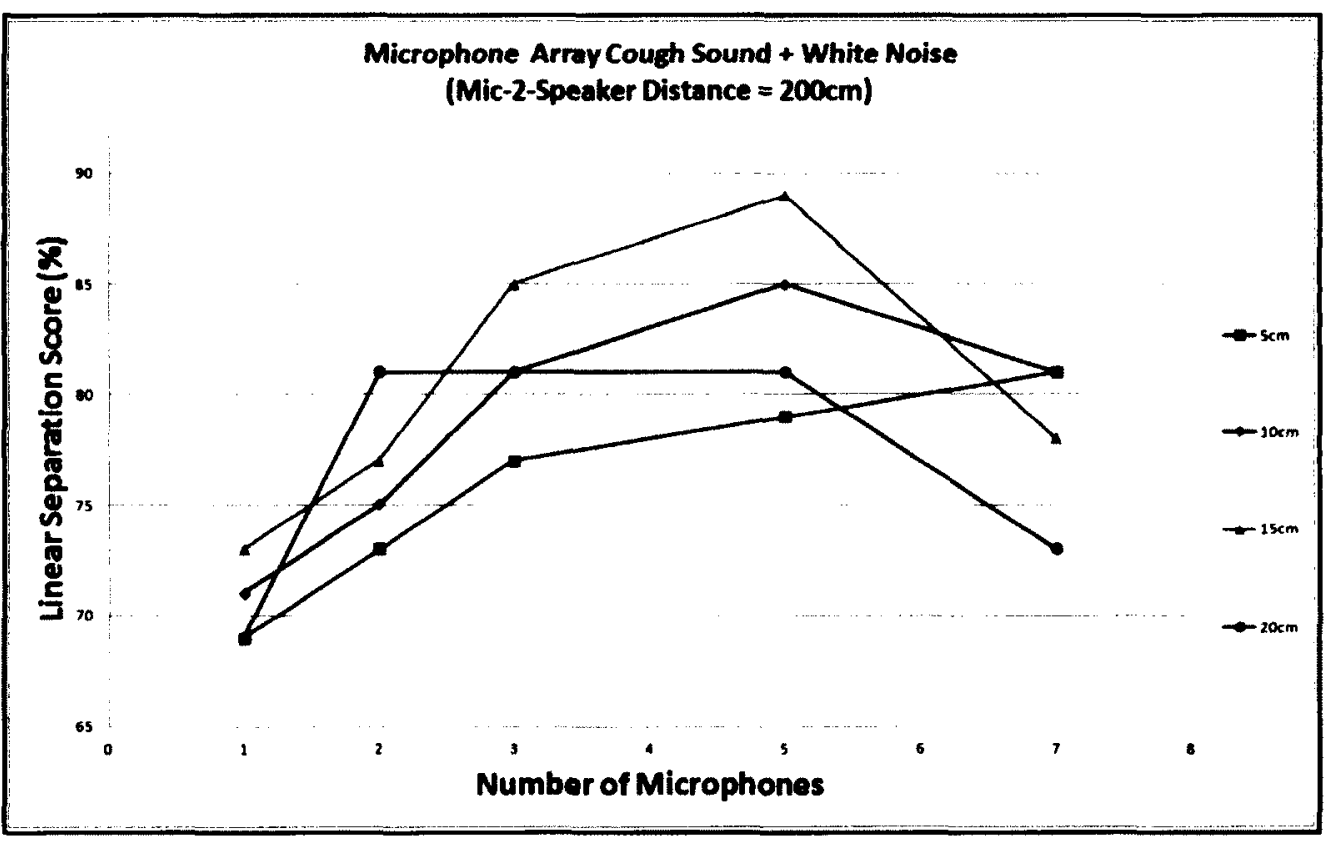

Figure 5.10: Microphone array cough sound acquisition and white noise $\left(D_{\text {Mic-2-Speaker }}=\right.$ $200 \mathrm{~cm})$. 


\subsection{Cough Sound Volume}

In the previous section, the performance of the cough sound discriminator was analysed in the presence of white noise. It was observed that the performance improves as the number of microphones increase. In this section, the effect of the cough sound volume is investigated.

As mentioned previously, cough sounds were played at $-10 \mathrm{~dB}$ levels. This level was selected because it did not cause any saturation for both the speaker and the microphones. In the presence of white noise, however, increasing the volume of cough might help with the performance of the cough sound discriminator.

In order to analyse the effect of cough sound volume, the cough sound acquisition was repeated with the following experimental setup: microphone-to-speaker distance of $200 \mathrm{~cm}$ and microphone-to-microphone distance of $15 \mathrm{~cm}$. The volume of the cough sounds were varied from $-20 \mathrm{~dB}$ to $8 \mathrm{~dB}$ and the acquired cough sounds were passed through the beamforming algorithm, followed by the cough sound discriminator and the Linear Separation Score was obtained. Figure 5.11 and Table 14 depict the results obtained in this section. As it could be seen from the results, increasing the volume of the cough sounds improved the performance of the cough sound discriminator. The performance, however, decreased at very high volumes due to speaker and microphone saturation that occurs at high cough sound volume levels. 
Table 14: LSS of microphone array cough sound acquisition with white noise and variable cough sound volume

\begin{tabular}{|c|c|c|c|c|c|c|c|}
\hline 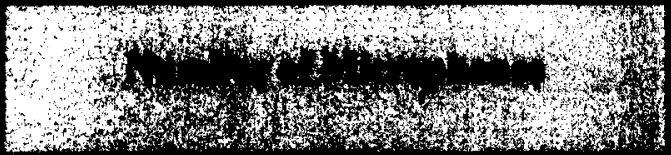 & & & & & & & sing \\
\hline 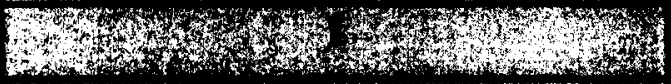 & 60 & 73 & 69 & 71 & 71 & 73 & \\
\hline 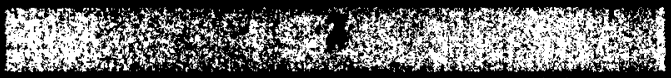 & 60 & 77 & 79 & 81 & 81 & 85 & 11 \\
\hline H. & 63 & $\mathbf{8 5}$ & 87 & 89 & 89 & 91 & 85 \\
\hline 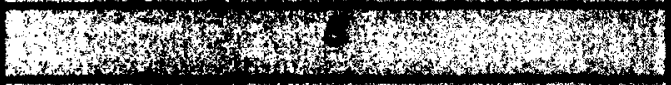 & 65 & 89 & 89 & 91 & 89 & 91 & 85 \\
\hline 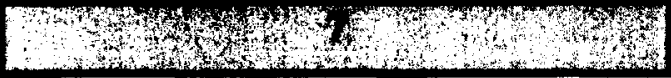 & 58 & 78 & 78 & 85 & 85 & $\mathbf{8 5}$ & 83 \\
\hline
\end{tabular}

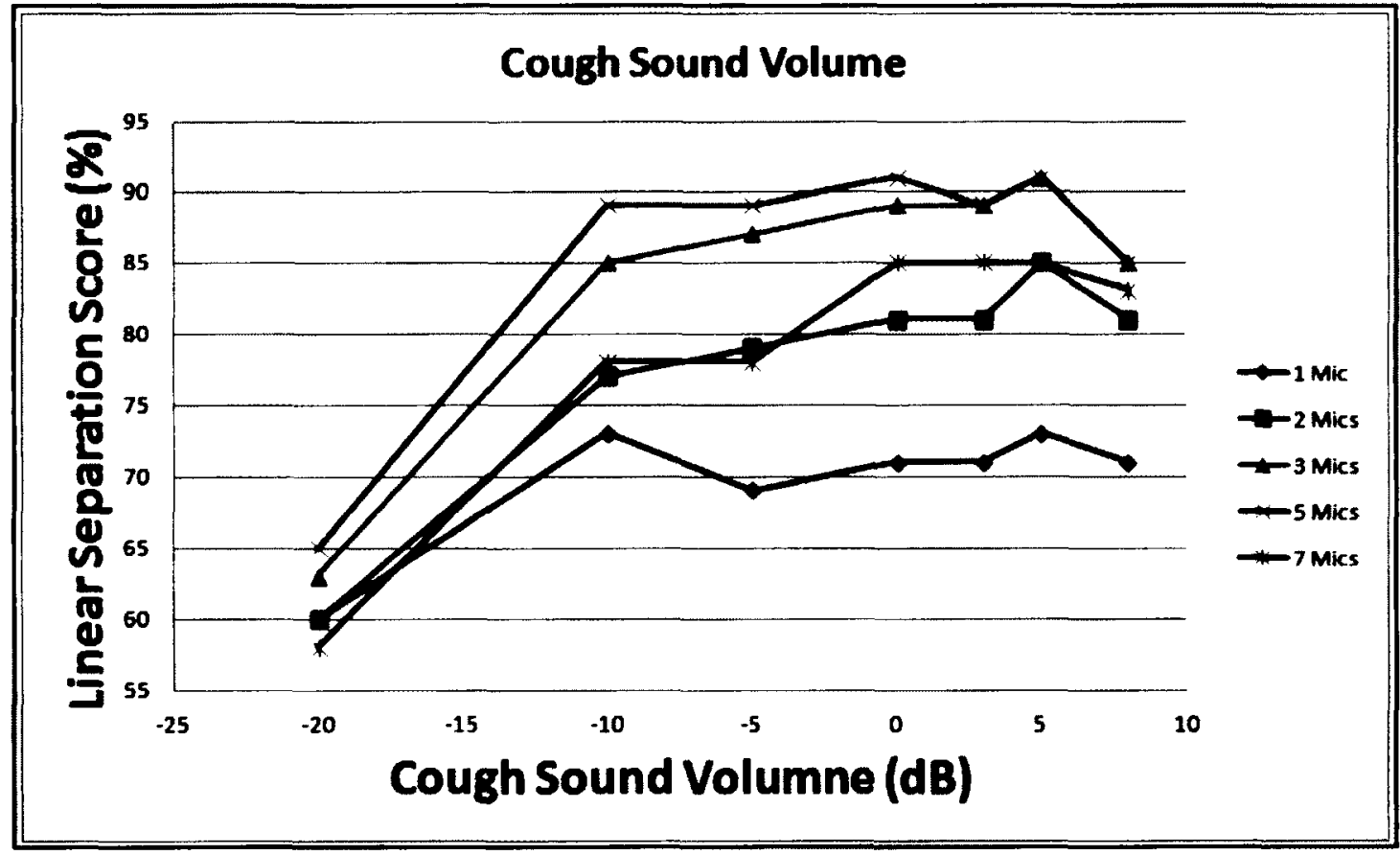

Figure 5.11: Microphone array cough sound acquisition with white noise and variable cough sound volume. 


\subsection{Discussions}

In this chapter, microphone array and beamforming techniques were used in order to improve the performance of the cough sound discriminator. In chapter 4 , the performance of the cough sound discriminator was evaluated using the Linear Separation Score developed in chapter 3 . The results obtained in the single-microphone cough sound acquisition revealed that the cough sound discriminator's performance decreases as the distance between the speaker and the microphone increases. Furthermore, the presence of white noise greatly reduces the Linear Separation Score.

In order to improve the performance of the cough sound discriminator, microphone arrays were used in the cough sound acquisition stage of the experiment. Furthermore, a delay-and-sum beamforming technique was used in order to combine the signals acquired by the individual microphones.

A delay-and-sum beamformer was selected due to its simplicity and ease of implementation. Furthermore, since the performance of the cough sound discriminator using microphone arrays was unknown, delay-and-sum beamformer provided an excellent starting point. More complex beamforming algorithms would potentially use the results obtained by the delay-and-sum beamformer as their reference and try to improve upon it [64][65]. The delay estimation of the delay-and-sum beamformer is the most critical component of this algorithm. For this reason, two methods were used in order to measure the relative delay between adjacent microphones. The reason for 
selecting two methods was to ensure the accuracy of the calculated delay. The first method was a mathematical calculation of the delay based on microphone-to-speaker and microphone-to-microphone distance. The second method, on the other hand, determined the delay using a simple experiment. The frequency of the tone used for delay calculation was selected to be much smaller than the sampling frequency of $44.1 \mathrm{kHz}$ in order to ensure minimal data loss between each sampling interval.

The microphone array used in the cough sound acquisition stage consisted of 1 to 7 microphones in total. Microphones were added to the array in such a way that it would preserve the symmetry of the setup. For this reason 1, 2, 3, 5 and 7 microphones were used in each experimental setup.

In the first part of the experiment, the effect of the microphone array on the performance of the cough sound discriminator was examined by varying the microphoneto-speaker distance and microphone-to-microphone distance. The distances used between the microphone array and the speaker were $150 \mathrm{~cm}, 200 \mathrm{~cm}$ and $250 \mathrm{~cm}$. These distances were selected due to the fact that closer distances of $50 \mathrm{~cm}$ and $100 \mathrm{~cm}$ used in the singlemicrophone cough sound acquisition resulted in an LSS score of 1 . Therefore, there was no need to use microphone array for those close distances. From the results depicted in Figure 5.5, Figure 5.6 and Figure 5.7, it could be seen that the performance of the cough sound discriminator improves when using more microphones. Adding more microphones and using beamforming techniques improves the signal-to-noise ratio (SNR) by 
amplifying the desired signal (i.e. cough) through in-phase addition of the desired signal and removing undesired signals through out-of-phase addition of the undesired signals. The overall performance improvement obtained for each distance varied. For $150 \mathrm{~cm}$ microphone-to-speaker distance, a maximum Linear Separation Score of 0.97 was obtained when using all 7 microphones at $15 \mathrm{~cm}$ microphone-to-microphone distance; this is a $7 \%$ improvement from the single-microphone cough sound acquisition. Similarly, for microphone-to-speaker distances of $200 \mathrm{~cm}$ and $250 \mathrm{~cm}$, a maximum LSS score of .93 and 0.85 was obtained respectively, as compared to 0.85 and 0.81 for the single-microphone case.

Although, adding more microphones improved the performance of the cough sound discriminator, the distance between the microphones also impacted the Linear Separation Scores. Figure 5.5, Figure 5.6 and Figure 5.7 revealed that the LSS obtained varied from different microphone-to-microphone distances. The distance between adjacent microphones within a microphone array affects the beampattern of the array and its ability to focus only on the desired signals and frequencies. From the figures, it could be seen that the microphone-to-microphone distances of $10 \mathrm{~cm}$ and $15 \mathrm{~cm}$ generally resulted in the greatest Linear Separation Score. According to Feature 2 of the cough sound discriminator described in [16], the highest frequency of interest is around $2250 \mathrm{~Hz}$. Therefore, the distance between the microphones within an array should not be less than $\lambda / 2$ [1]. The minimum distance could be calculated as shown in (8). As it could be seen 
from (8), the theoretical distance is less than the optimal distance obtained in the microphone array cough sound acquisition experiments.

$$
d \geq \lambda / 2=v / 2 f=7.6 \mathrm{~cm}
$$

In the second part of this chapter, the effect of microphone array on the performance of the cough sound discriminator was investigated in the presence of white noise. It was observed in section 4.4 that the performance of the cough sound discriminator significantly decreases with single-microphone cough sound acquisition in the presence of white noise. In this section, the single microphone was replaced by a microphone array in order to improve the previously obtained Linear Separation Score. Figure 5.9 and Figure 5.10 depict the results obtained. From the two figures, it could be seen that a significant improvement was observed when using a microphone array as opposed to a single microphone. For the microphone-to-speaker distance of $50 \mathrm{~cm}$, a maximum Linear Separation Score of 0.95 was obtained as opposed to 0.81 in the single-microphone cough sound acquisition. For the microphone-to-speaker distance of $200 \mathrm{~cm}$, a maximum Linear Separation Score of 0.89 was obtained as opposed to 0.69 in the singlemicrophone cough sound acquisition. This significant improvement is due to the fact that microphone array and delay-and-sum beamforming algorithm provides the ability to cancel unwanted noise while amplifying desired signals. For the $50 \mathrm{~cm}$ microphone-to- 
speaker distance, $5 \mathrm{~cm}$ and $10 \mathrm{~cm}$ microphone-to-microphone distance resulted in the maximum Linear Separation Score. For the $200 \mathrm{~cm}$ microphone-to-speaker distance, however, the $15 \mathrm{~cm}$ microphone-to-microphone distance resulted in the best performance. These findings could be explained by the beampattern formed by each of the configurations and also by the number and location of side-lobes which exist in each configuration. The beampattern and shape will be explained and depicted graphically in the next chapter.

Figure 5.10 also reveals another interesting finding. At microphone-to-microphone distances of $15 \mathrm{~cm}$ and $20 \mathrm{~cm}$, it is observed that the LSS score significantly decreases when using 7 microphones. Although this observation initially seems non-intuitive, it could be explained by taking a closer look at the experimental setup. From Figure 5.8, it could be observed that microphone number 7 is the closest microphone to the white noise source. As the distance between the microphones increase, microphone number 7 gets closer to the white noise source. At microphone-to-microphone distances of $15 \mathrm{~cm}$ and 20 $\mathrm{cm}$, microphone number 7 is so close to the white noise source that two events occurs. First, the microphone is picking up only white noise and barely any of the actual desired signals. This causes the delay used in the delay-and-sum beamforming algorithm to lose its significance and cause noise to also be added to the desired signal. In this case, the microphone is doing more harm than good and hence decreases the performance of the cough sound discriminator. Second, being so close to the white noise source, which is 
being played at a certain volume level, causes the microphone to saturate and hence not capture the desired signals appropriately. These two factors contribute to the fact that adding the $7^{\text {th }}$ microphone decreases the overall LSS score.

In the final part of this chapter, the volume of the cough sounds were varied in order to determine the effect of cough sound volume on the performance of the cough sound discriminator in the presence of white noise. Cough sounds volumes were varied from $-20 \mathrm{db}$ to a maximum of $8 \mathrm{~dB}$ for a microphone-to-speaker distance of $200 \mathrm{~cm}$ and a microphone-to-microphone distance of $15 \mathrm{~cm}$. From the result depicted in Figure 5.11, it could be seen that increasing the volume of the cough sounds improves the Linear Separation Score which is due to an increase in the signal-to-noise ratio. Furthermore, adding more microphones to the microphone array also improves the performance of the cough sound discriminator. The increase in volume at very high levels however, decreases the performance of cough sound discriminator. This is due to the fact that at very high volumes, the various components of the system such as the microphones and the speakers enter saturation levels and start injecting noise into the system. This injected noise and saturation leads to an overall decrease in the performance of cough sound discriminator.

\subsection{Conclusions}

In this chapter, microphone arrays were used in order to improve the performance of the cough sound discriminator in noisy and reverberant environments. Furthermore the effect 
of cough sound volume on the performance of the cough sound discriminator was investigated. From the results obtained in this chapter, it was concluded that adding more microphones improve the Linear Separation Score in both noisy and reverberant environments. Furthermore, increasing the volume of the cough sounds improves the overall performance of the cough sound discriminator.

The use of microphones arrays for cough sound acquisition is a necessary step in improving the performance of the cough sound discriminator. In chapter 4 , it was concluded that the performance of the cough sound discriminator significantly decreases as the distance between microphone and speaker increase and also as white noise is added to the system. This decrease in performance was compensated by using microphone arrays in this chapter. Microphone arrays and delay-and-sum beamforming techniques are capable of focusing on the desired signals while removing unwanted signals and noise from the final cough signal. 


\section{Chapter 6:}

\section{Microphone Array and Beamforming}

\section{Graphical User Interface}

Microphone array and delay-and-sum beamforming algorithm was used in order improve the performance of the cough sound discriminator and its overall Linear Separation Score. In order to understand the behaviour of the microphone array and its beampattern, a Graphical User Interface (GUI) was designed. The design of this GUI is explained in details in this chapter. Furthermore, the results obtained in chapter 5 will be illustrated and analysed in this chapter using the GUI.

This chapter starts by giving an overview of the developed GUI along with its various features. The algorithms used in developing the GUI and displaying the beampattern are then explained. Finally, the results obtained in chapter 5 are illustrated using the GUI. 


\subsection{Introduction}

The GUI designed in this section was used in order to visualise the beampattern of various microphone array configurations. The shape of the emitted beampattern and the presence of side-lobes could help with understanding the results obtained in chapter 5 .

The relationship between the number of microphones, the frequency of the sound source, the distance between the microphone array and the sound source and the distance between the microphones within the array on the final beampattern could be simulated using the GUI. The knowledge of the approximate beampattern helps with understanding the performance of the cough sound discriminator in various situations.

\subsection{Graphical User Interface Design}

The graphical user interface was developed in Matlab. Figure 6.1 shows a snapshot of the GUI when it is started for the first time. As it can been seen from the figure, it consists of two major parts: The open grey area which is a placeholder for the beampattern diagram and the bottom part which is the control area. 


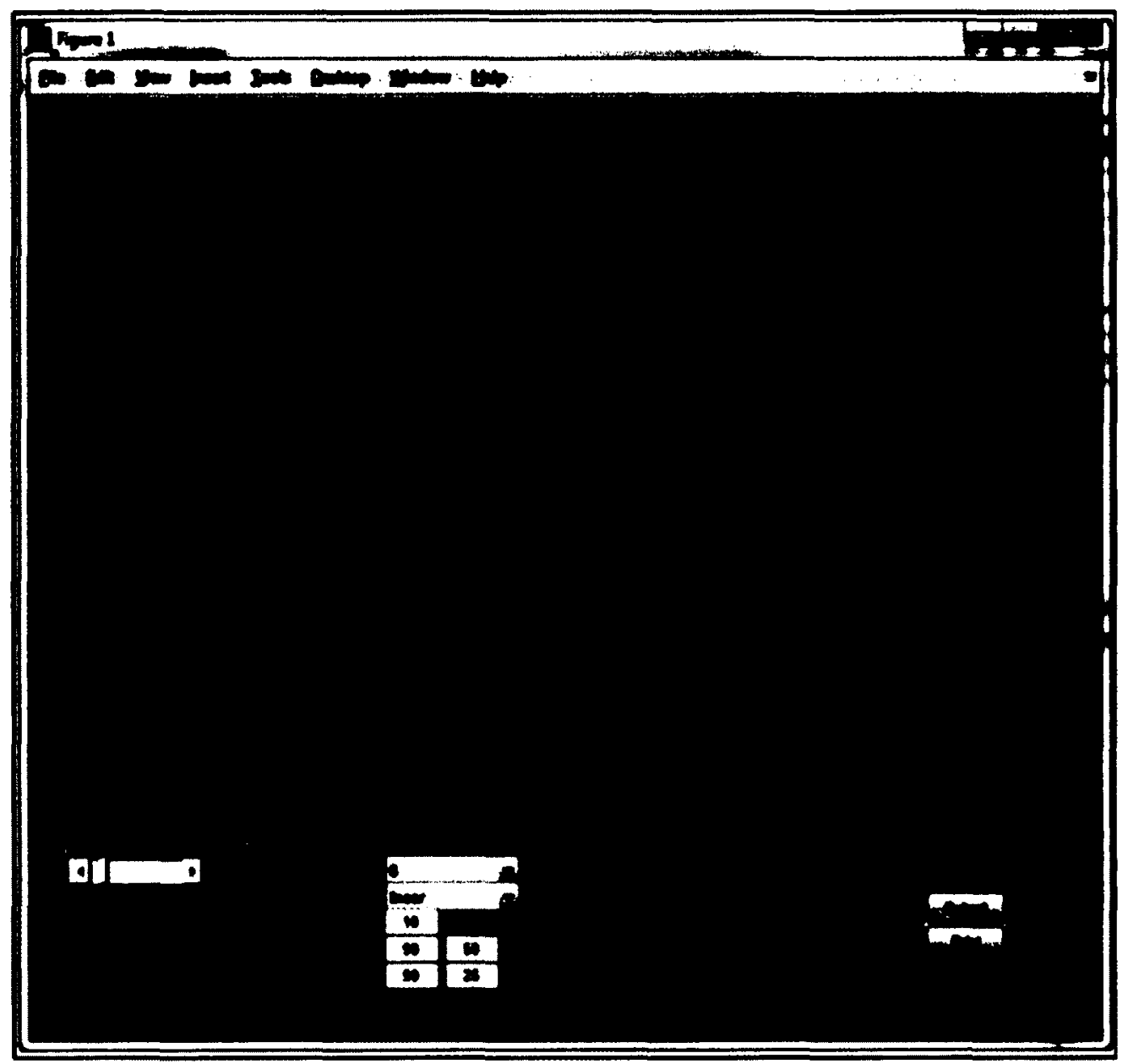

Figure 6.1: Graphical User Interface.

The control area depicted in Figure 6.1 gives the user the ability to set the various parameters for a given microphone array setup. The users are capable of modifying the frequency of sound source using the frequency slider, and setup the various components of the microphone array configuration such as the number of microphones, its arrangement, microphone-to-microphone distance and the coordinates of the sound 
source and microphones. Once the configuration is setup, the "submit" button is used to generate the corresponding beampattern and the "print" button is used to capture a screenshot of the beampattern. The microphones could be arranged in a linear or random fashion. In linear mode, the user only needs to specify the coordinate of the center of the microphone array and the distance between the microphones and the program calculates all the coordinates of the microphones automatically. In manual mode, however, the user is capable of manually placing the microphone in any location they desire. In manual mode, a new control pane becomes visible as shown in Figure 6.2. Figure 6.3 depicts a sample output of the GUI. The red square represents the sound source and the blue squares represent the microphone array. Darker regions represent greater signal attenuation and lighter regions represent less signal attenuation.

The beampattern diagram created by the GUI is a greyscale image of the signal amplitudes at various locations of a $2 \mathrm{~m} \times 2 \mathrm{~m}$ room. The signal amplitude calculation is explained in the next section.

\subsection{Beampattern and Delay-and-Sum Beamformer}

The algorithm used in the GUI is a delay-and-sum beamformer. The algorithm uses the coordinates of the sound source and microphones in order to compute the distance of each microphone from the sound source. The delay is then calculated by finding the longest microphone-to-sound source distance and adjusting all other distances relative to the longest distance. Once the delay for each microphone is calculated, the algorithm 
iteratively calculates the delay between each point on the graph and the microphones and sums all the signals captured by the microphones. The value obtained is normalized to a value between 0 and 1 , which is used for the greyscale drawing.

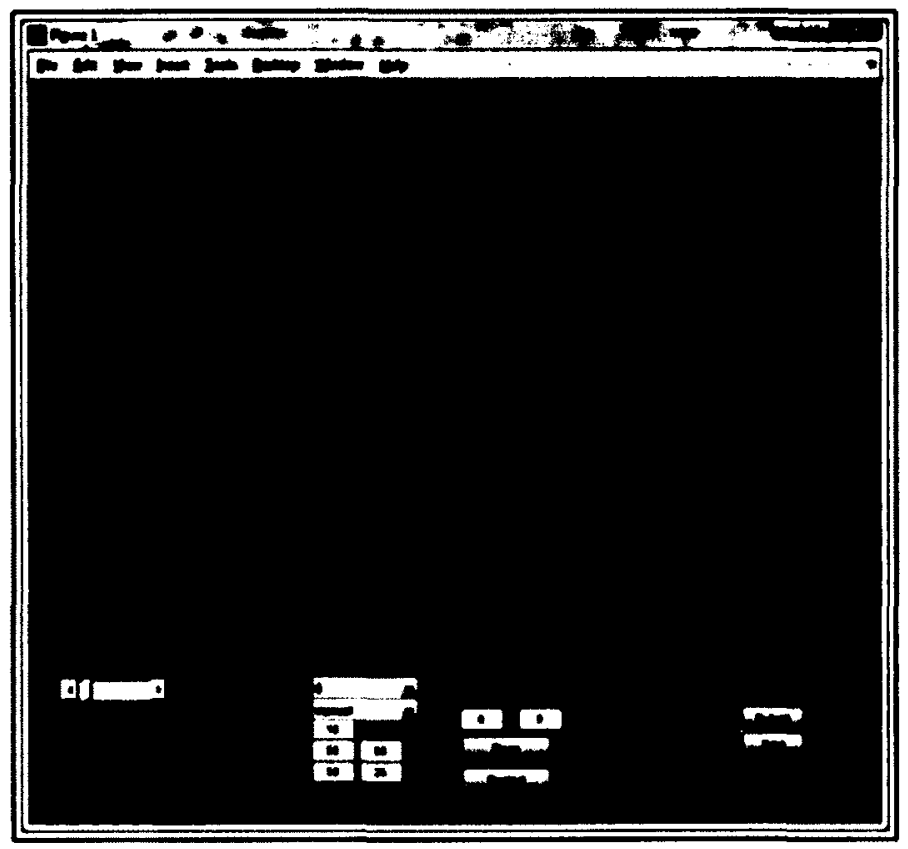

Figure 6.2: Manual microphone placement pane. 


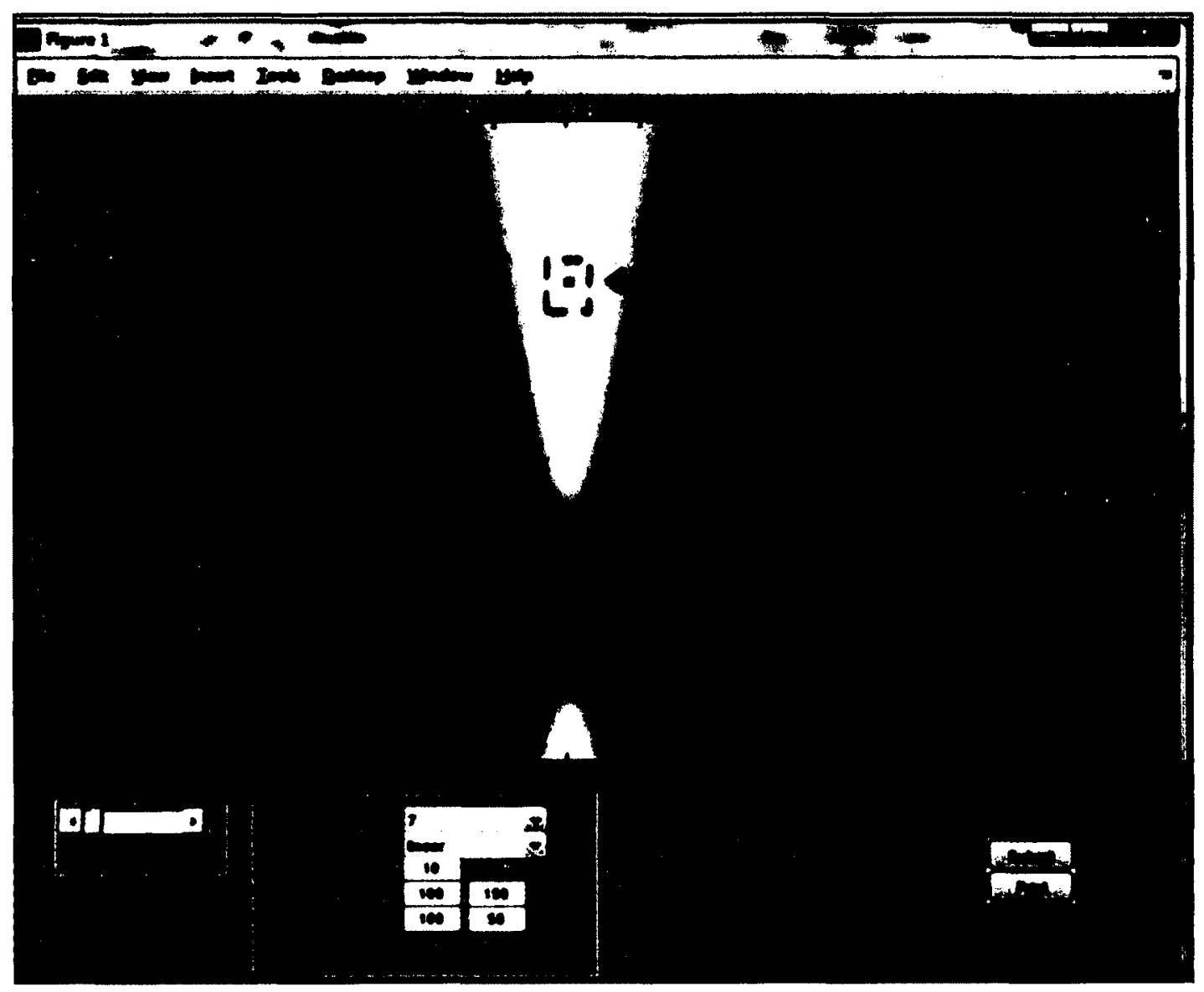

Figure 6.3: Sample beampattern diagram (linear mode).

\subsection{Beampattern Analysis}

In this section, the beampattern of the various microphone array configurations used in chapter 5 are simulated in order to further analyse the results obtained using microphone array and delay-and-sum beamforming. The frequency used for the simulation is 2000 
$\mathrm{kHz}$, which is roughly the maximum frequency used in the cough discrimination algorithm.

\subsubsection{Microphone-to-Speaker Distance}

In this section, the effect of microphone-to-speaker distance on the generated beampattern is simulated using a microphone array of seven microphones with a $10 \mathrm{~cm}$ microphone-to-microphone distance. As it can be seen from Figure 6.4, Figure 6.5, Figure 6.6, and Figure 6.7 as the distance between the microphone and speaker increases, the beam width also increases. This widening of the beam width enables the microphones to collect sound signals from sources other than the desired source and hence reduce the signal-to-noise ratio.

\subsubsection{Microphone-to-Microphone Distance}

In this section, the effect of microphone-to-microphone distance and the number of microphones on the shape of the beampattern is simulated using the GUI.

Figure 6.8, Figure 6.9, Figure 6.10 depict the beampattern as more microphones are added to the microphone array. As it could be seen, adding more microphones creates a narrower beam towards the desired sound source. Although a narrower beampattern helps focus on the desired signal, it is also accompanied by additional side-lobes. The side- 
lobes will pick up any noise that might be present where the side-lobes exist, which in turn would decrease the signal-to-noise ratio.

The results obtained in section 5.3 revealed that a microphone-to-microphone distance of $10 \mathrm{~cm}$ and $15 \mathrm{~cm}$ resulted in the highest Linear Separation Score. By examining the simulation results in Figure 6.11, Figure 6.12 and Figure 6.13 it could be seen that for frequencies of $2 \mathrm{kHz}$, the microphone-to-microphone distance of $10 \mathrm{~cm}$ and $15 \mathrm{~cm}$ results in both a narrow beam and a relatively smaller side-lobe than that of $5 \mathrm{~cm}$ or $20 \mathrm{~cm}$. This pattern could be better observed when using more microphones in the microphone array and in the presence of noise. As a result, the signal-to-noise ratio for these microphone-to-microphone distances was higher than other microphone array configuration. The higher the signal-to-noise ratio, the higher the Linear Separation Score of the cough sound discriminator. 


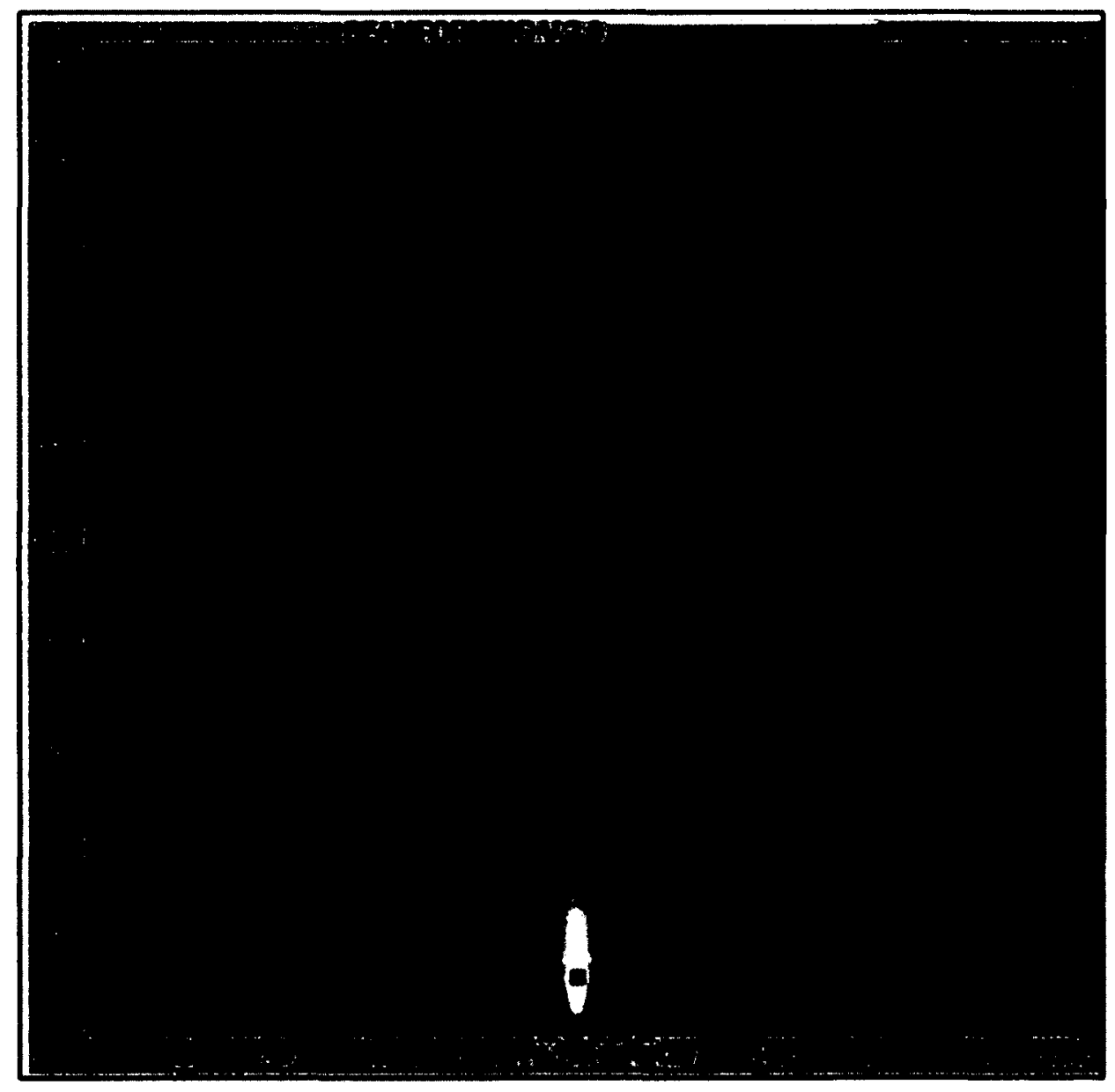

Figure 6.4: Beampattern for $D_{\text {Mk-to-Speaker }}=10 \mathrm{~cm}$. 


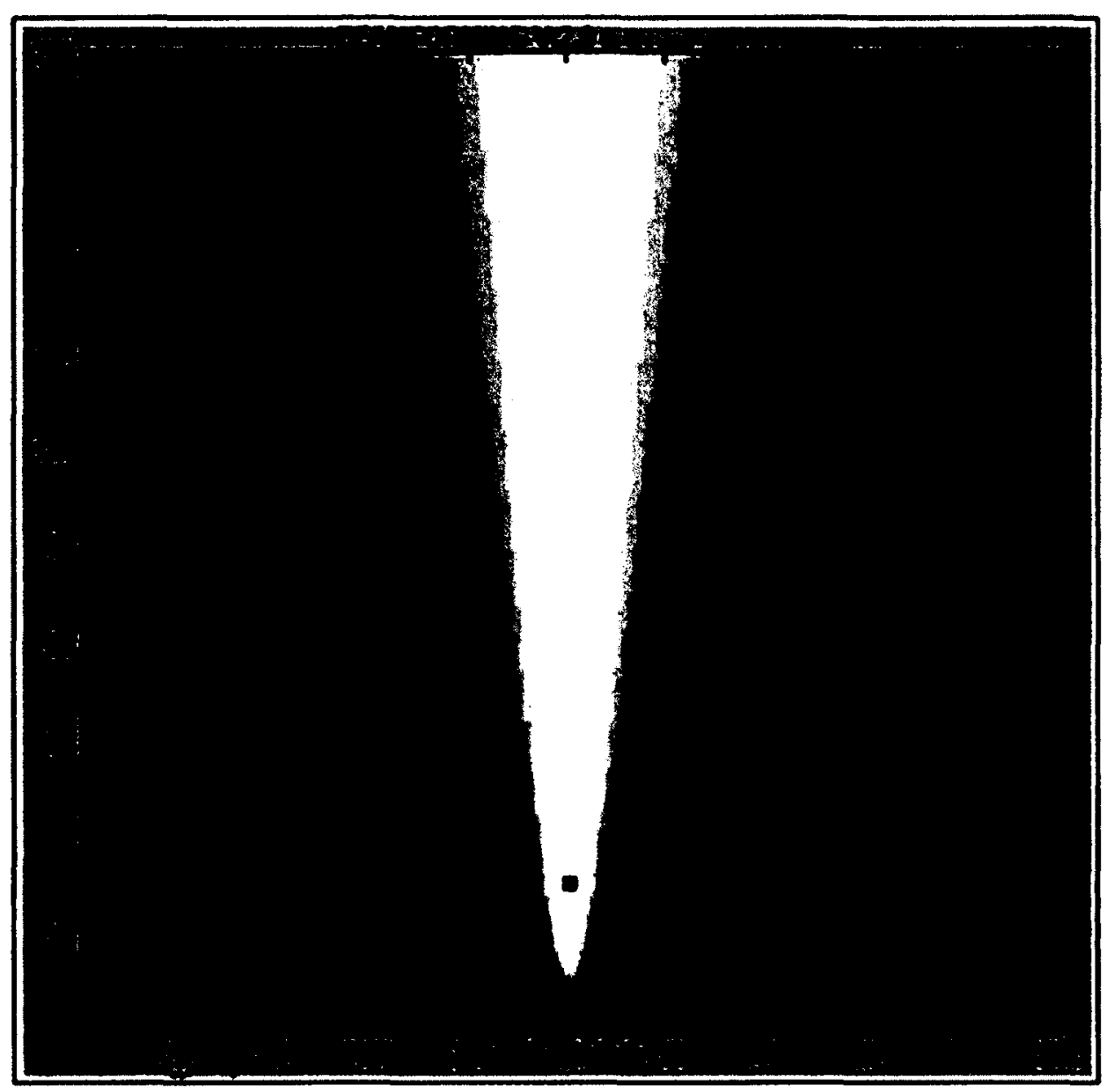

Figure 6.5: Beampattern for $D_{\text {Mik-10-Speaker }}=30 \mathrm{~cm}$. 


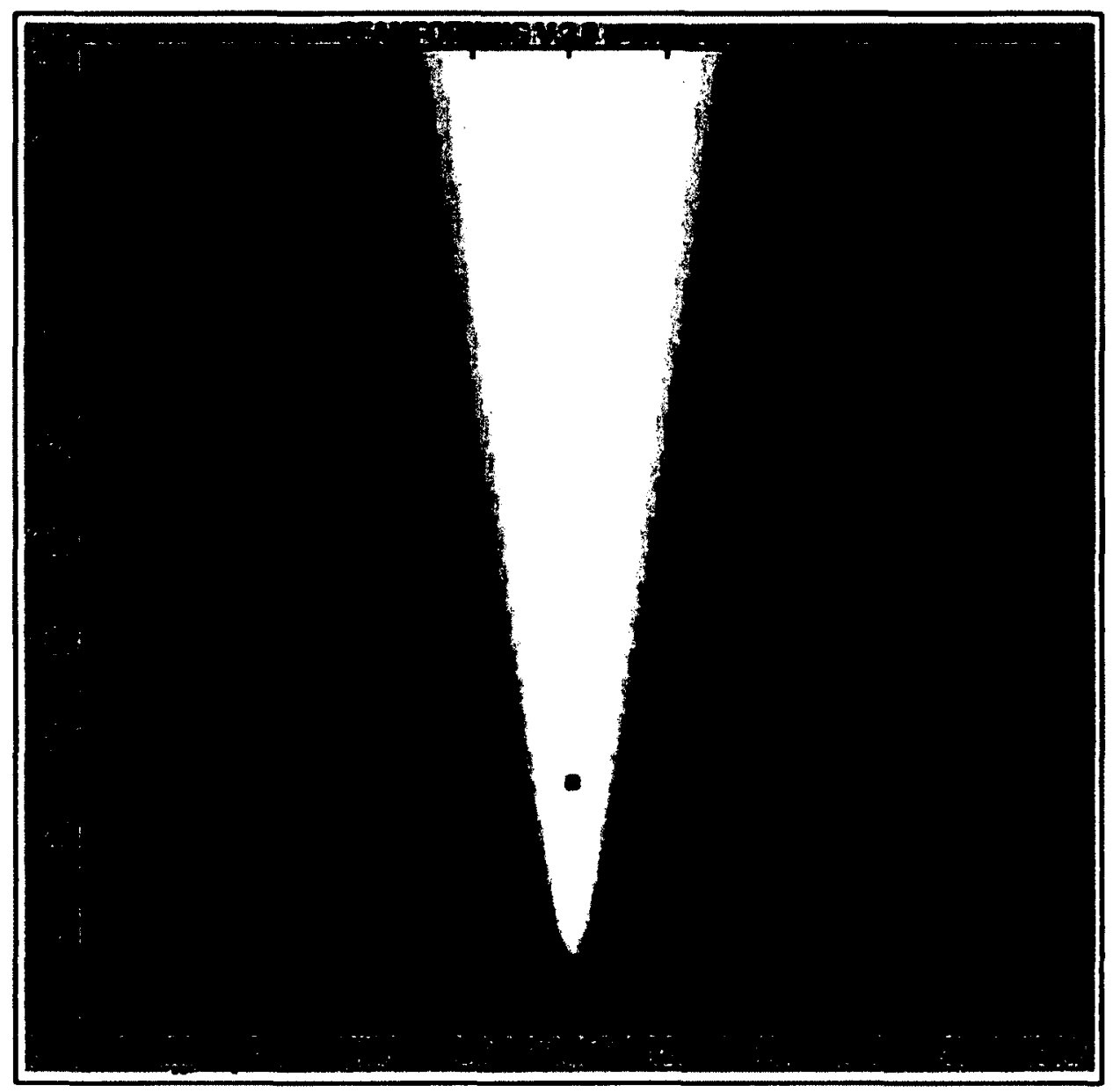

Figure 6.6: Beampattern for $D_{\text {Mr-to-Speaker }}=50 \mathrm{~cm}$. 


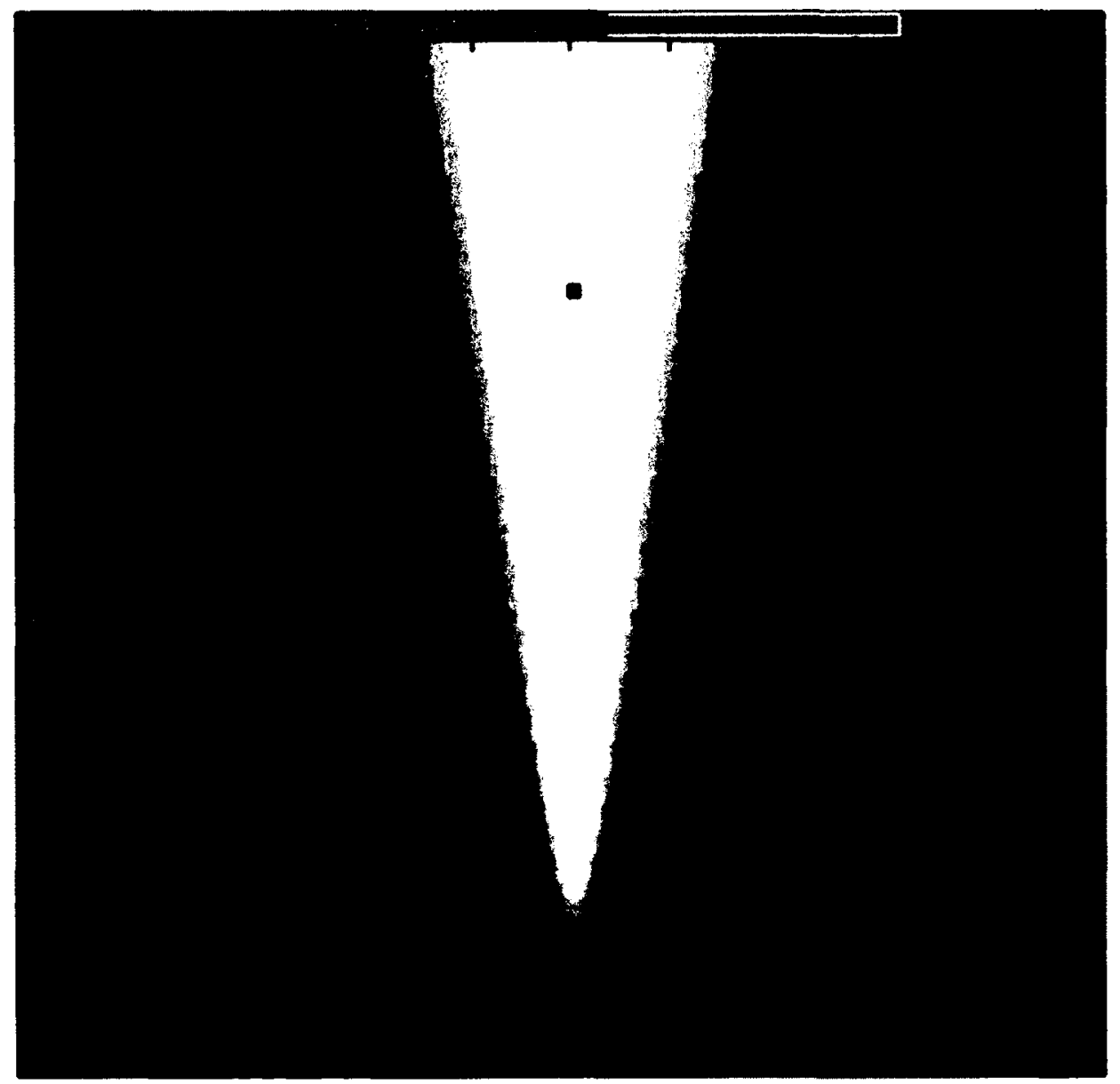

Figure 6.7: Beampattern for $D_{\text {Mik-to-Speaker }}=150 \mathrm{~cm}$. 


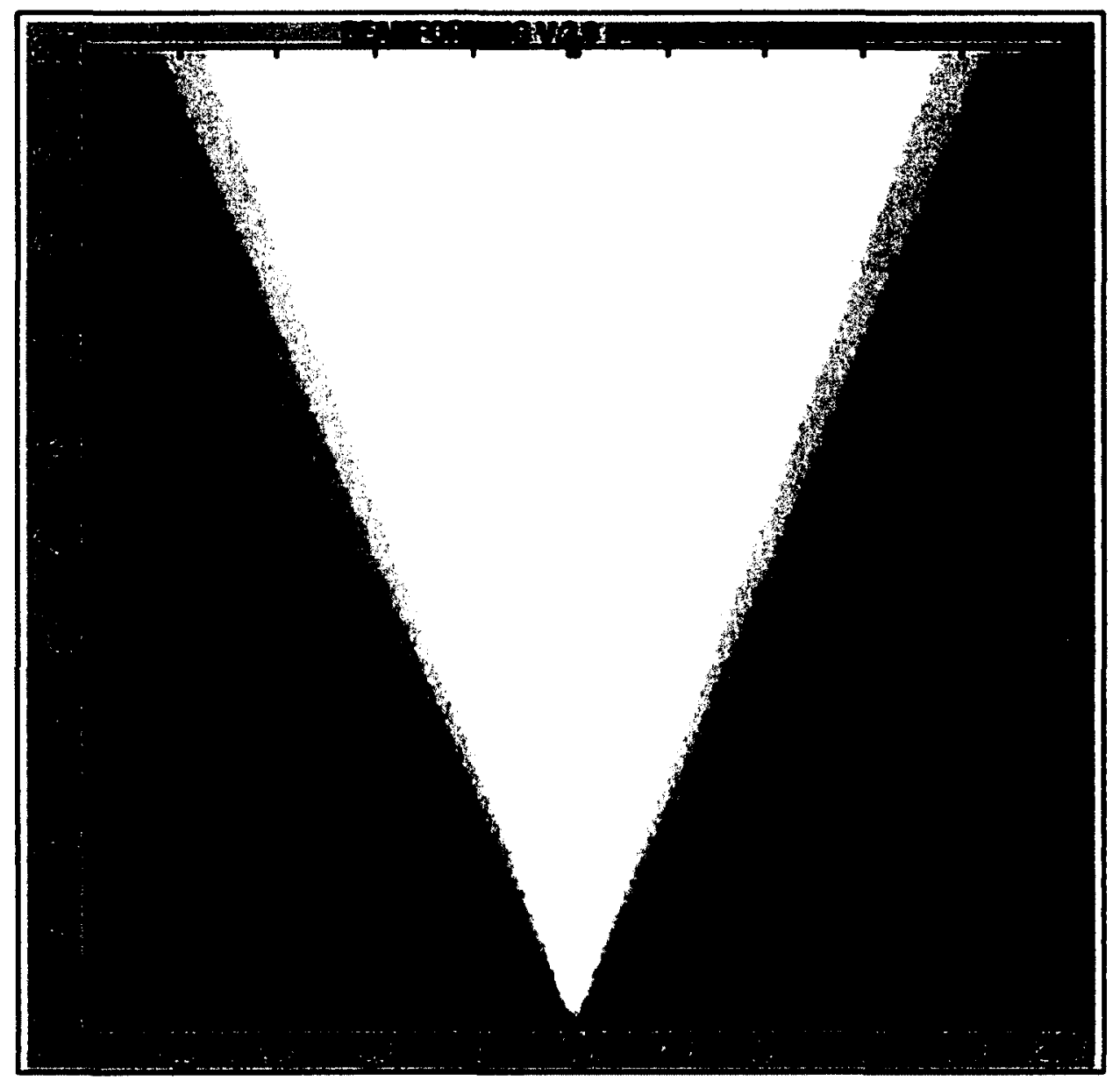

Figure 6.8: Beampattern using 3 microphones. 


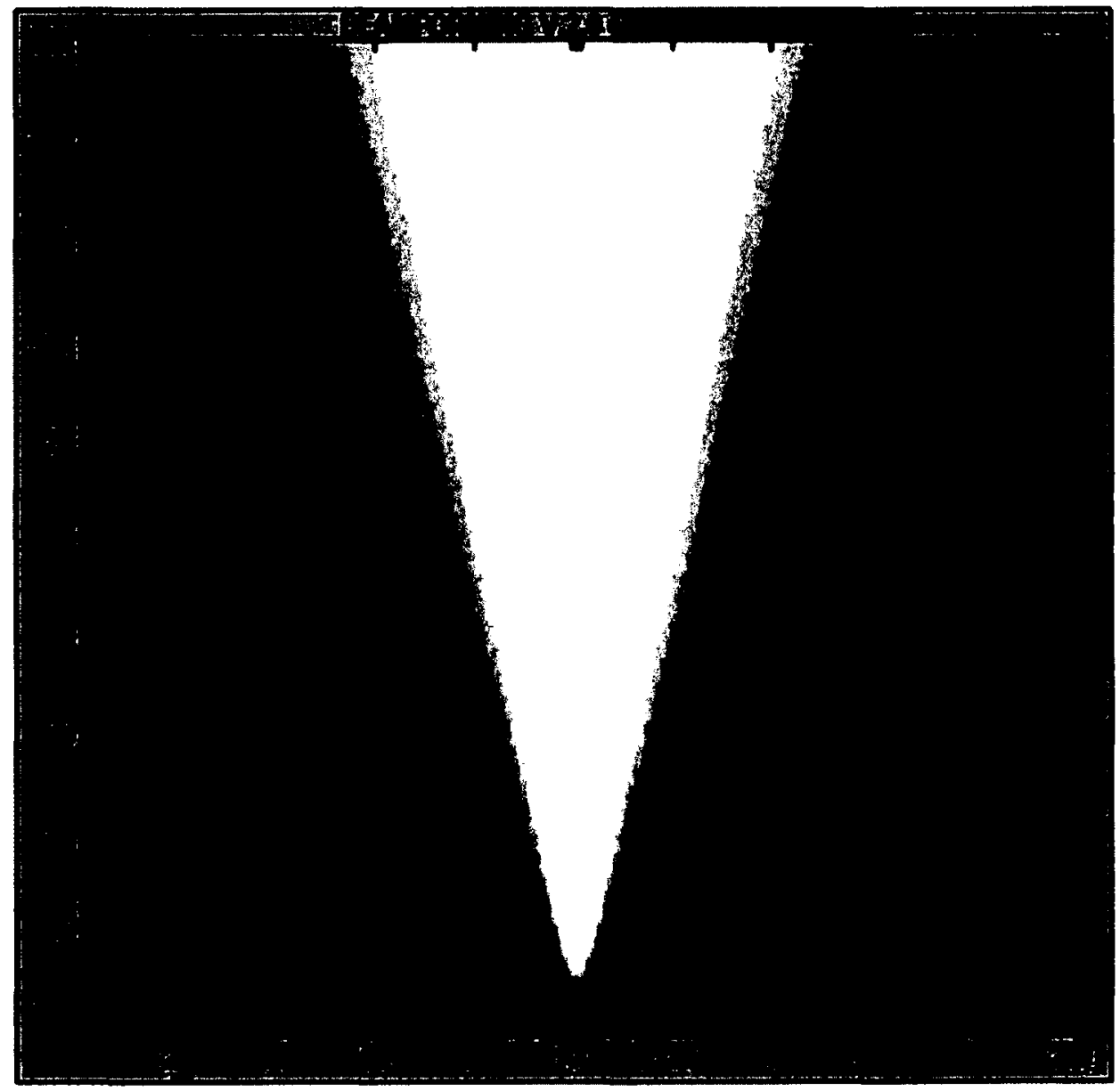

Figure 6.9: Beampattern using 5 microphones. 


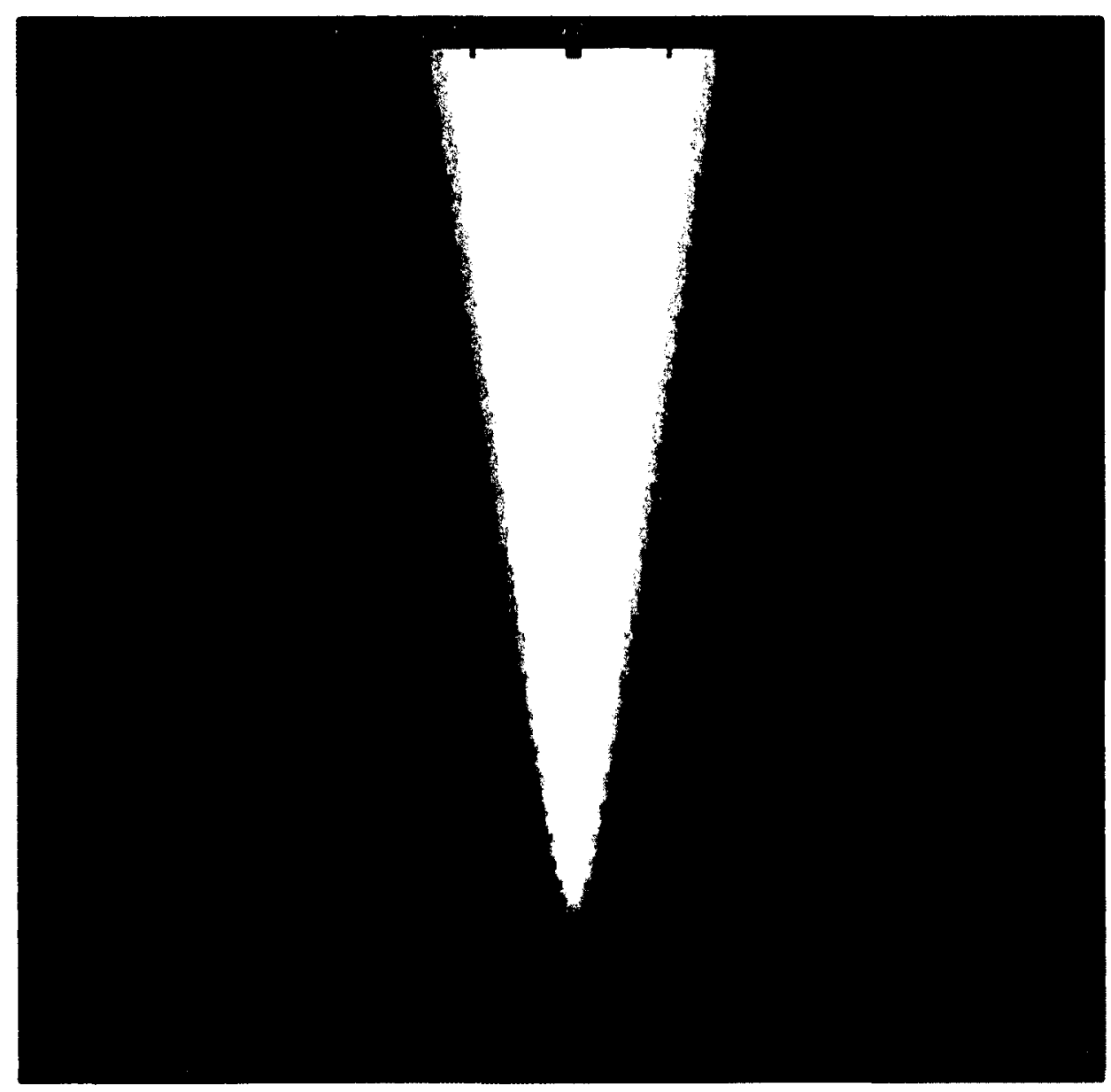

Figure 6.10: Beampattern using 7 microphones. 


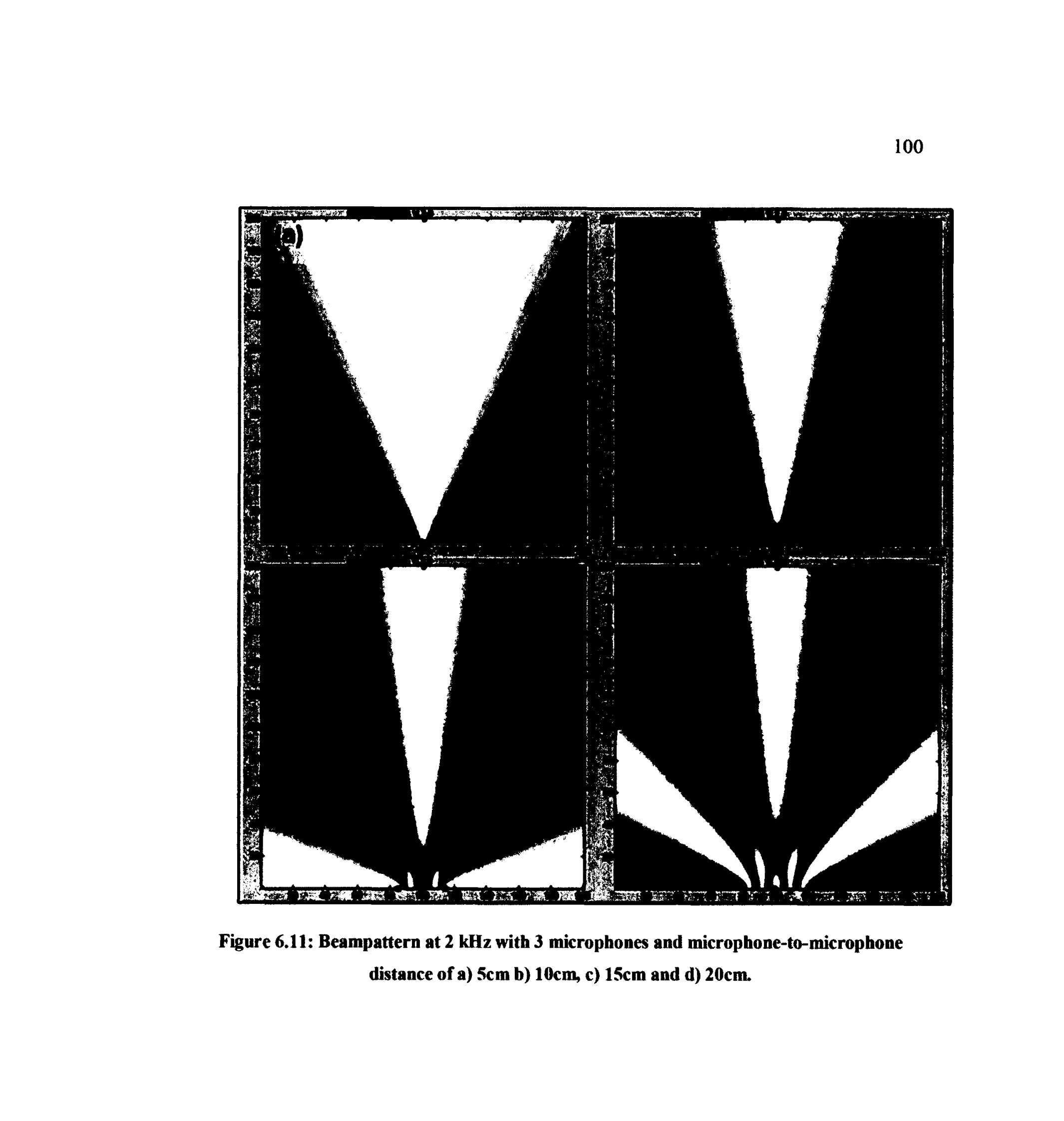




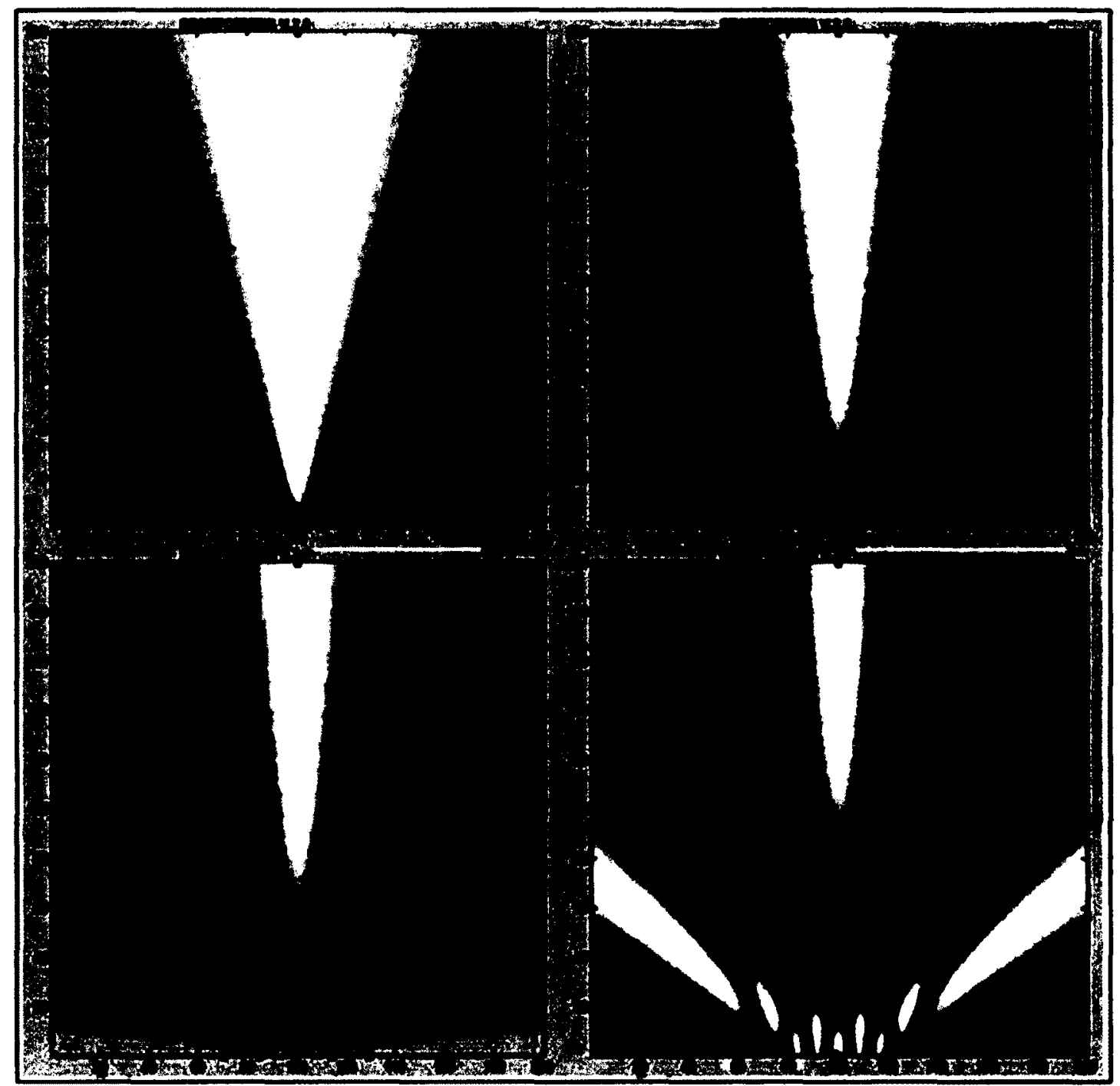

Figure 6.12: Beampattern at $2 \mathrm{kHz}$ with 5 microphones and microphone-to-microphone distance of a) $5 \mathrm{~cm} \mathrm{b)} 10 \mathrm{~cm}$, c) $15 \mathrm{~cm}$ and d) $20 \mathrm{~cm}$. 


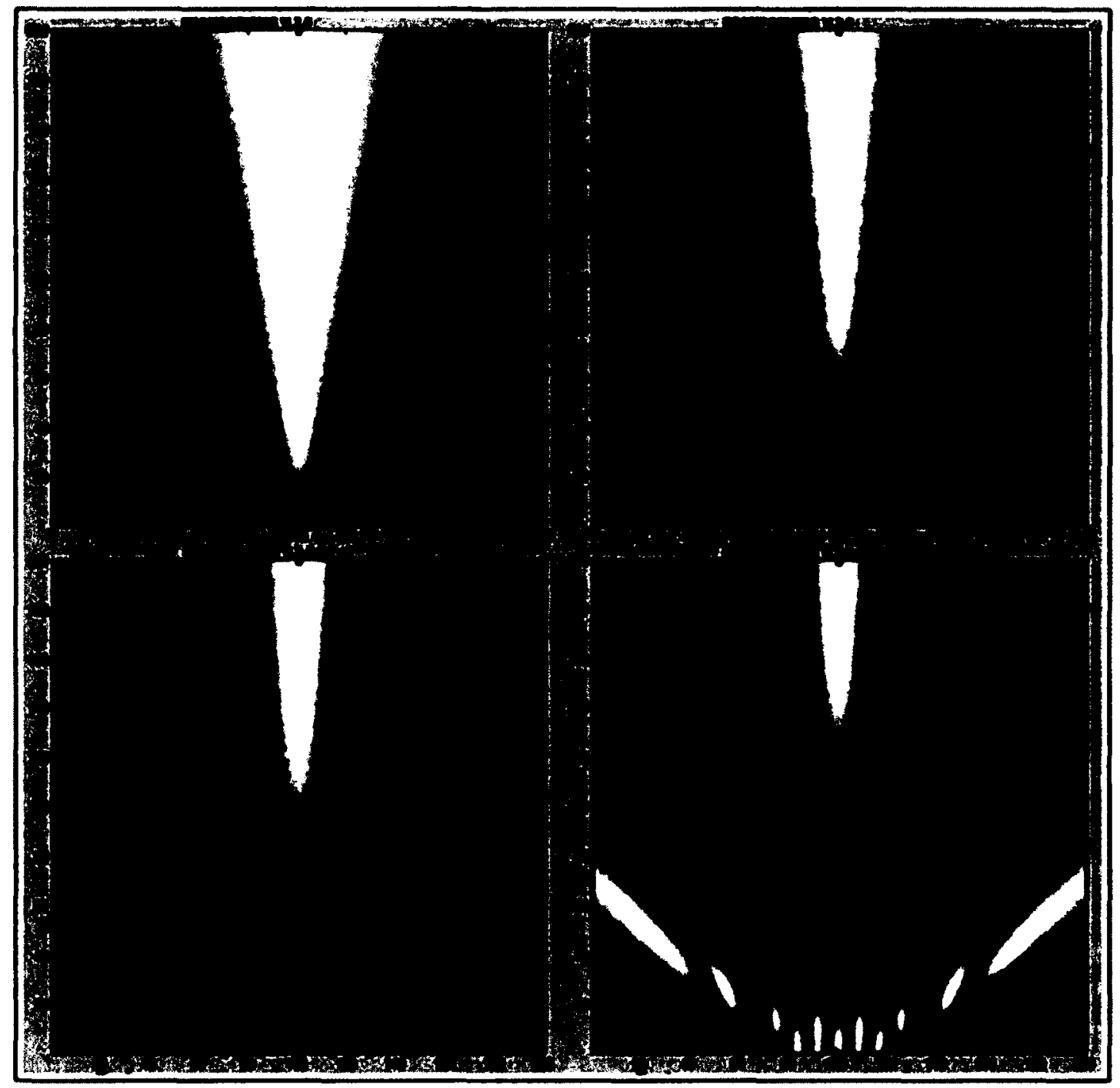

Figure 6.13: Beampattern at $2 \mathrm{kHz}$ with 7 microphones and microphone-to-microphone distance of a) $5 \mathrm{~cm}$ b) $10 \mathrm{~cm}$, c) $15 \mathrm{~cm}$ and d) $20 \mathrm{~cm}$. 


\subsection{Conclusions}

In this chapter, the design of a Graphical User Interface was explained. The GUI was developed in order to simulate the beampattern obtained by various microphone array configurations. Delay-and-sum beamforming algorithm was used in the GUI in order to compute the beampattern of the microphone.

The GUI was used in order to simulate the configurations used in chapter 5 and help analyse the results. The simulation results showed that as the number of microphones increased, a narrower beam is formed towards the desired sound source. Furthermore, as the distance between the microphones increased, the beam also became narrower and more side-lobes were formed by the beamformer. The existence of side-lobes could significantly reduce the performance of the beamformer if the location of the noise coincides with the location of the side-lobe.

In conclusion, the simulation results obtained in this chapter helped explain the findings obtained in chapter 5 . 


\section{Chapter 7:}

\section{Conclusion}

\subsection{Thesis Conclusion and Contributions}

In this thesis, the cough sound discriminator previously developed by [16] was analysed in noisy and reverberant environments. These types of environments were selected since they represent the likely environments in which patients are monitored. In order to measure the performance of the cough sound discriminator, Linear Separation Score (LSS) was introduced. Linear Separation Score used linear separability of the wet and dry cough sounds in order to quantitatively compare the performance of the cough sound discriminator under various conditions.

The performance of the cough sound discriminator was analysed in a noisy and reverberant room using a single microphone. Experiments revealed that the Linear 
Separation Score decreased as the distance between the microphone and the speaker increased. Furthermore, the addition of white noise significantly decreased the performance of the cough sound discriminator. In order to improve the Linear Separation Score of the cough discriminator, microphones arrays were used along with delay-andsum beamforming algorithm. Results showed that using microphone arrays improved the performance of the cough sound discriminator in both reverberant and noisy environment. The effect of microphone arrays on the performance of the cough sound discriminator was fully analysed under various experimental setups such as microphoneto-microphone distance, microphone-to-speaker distance, the number of microphones and the volume of the cough sounds.

Finally, a Graphical User Interface was developed using Matlab in order to help simulate the beamforming algorithm and illustrate the beampattern created by the microphone array under different configurations. The simulation results obtained by the GUI were used to explain the results obtained in the microphone array experimentations.

\subsection{Suggested Future Work}

Future work relevant to the research performed in this thesis includes:

- Use other types of beamforming algorithms in order to further improve the performance of the cough sound discriminator.

- Develop new feature extraction algorithms capable of discriminating between dry and wet cough sounds under noisy and reverberant environments 
- Develop a cough sound classifier

- Expand the cough database to include more cough sounds

- Add more features to the GUI such as flexible room size, add other beamforming algorithms and analyse more complex sound signals 


\section{References}

[1] J. Benesty, J. Chen, and Y. Huang, Microphone Array Signal Processing, Berlin: Springer, 2008.

[2] S. Haohai, H. Teutsch, E. Mabande, and W. Kellermann, "Robust localization of multiple sources in reverberant environments using EB-ESPRIT with spherical microphone arrays," in Proc. IEEE International Conference on Acoustics, Speech, and Signal Processing (ICASSP), Prague, Czech Republic, May 2011, pp. 117-120.

[3] J. Weisen, C. Zebin, L. Meifeng, and Y. Zhu Liang, "A simple microphone array for source direction and distance estimation," in Proc. $6^{\text {th }}$ IEEE Conference on Industrial Electronics and Applications $(A C I E A)$, Beijing, China, Jun. 2011, pp. 1002-1005.

[4] H. Jwu-Sheng, T. Chieh-Min, C. Chen-Yu, and C. Yung-Jung, "Geometrical arrangement of microphone array for accuracy enhancement in sound source localization," in Proc. $8^{\prime \prime}$ Asian Control Conference (ASCC), Kaohsiung, Taiwan, May 2011, pp. 299-304.

[5] Y. Sasaki, S. Kagami, H. Mizoguchi, and T. Enomoto, "A predefined command recognition system using a ceiling microphone array in noisy housing environments," in Proc. IEEE/RSV International Conference on Intelligent Robots and Systems, Nice, France, Sep. 2008, pp. 2178-2184.

[6] C. Marro, Y. Mahieux, and K.U. Simmer, "Analysis of noise reduction and deverberation techniques based on microphone arrays with postfiltering," IEEE Transactions on Speech and Audio Processing, vol. 6 , issue. 3 , pp. $240-259$, 1998 .

[7] M. Cobos, J.J. Lopez, and S. Spors, "Analysis of room reverberation effects in source localization using small microphone arrays," in Proc. $4^{\text {th }}$ International Symposium on Communications, Control and Signal Processing (ISCCSP), Limassol, Cyprus, Mar. 2010, pp. 1-4.

[8] M. Hamalainen, and V. Myllyla, "Acoustic echo cancellation for dynamically steered microphone array systems," in Proc. IEEE Workshop on Applications of Signal Processing to Audio and Acoustics, New York, USA, Oct. 2007, pp. 58-61.

[9] T. Yensen, J. Ryan, R.A. Goubran, and I. Lambadaris, "Synthetic stereo acoustic echo cancellation structure with microphone array beamforming for VoIP conferences," in Proc. IEEE International Conference on Acoustics, Speech, and Signal Processing, Istanbul, Turkey, Jun. 2000, vol. 2, pp. I18 $17-I 1820$.

[10]A. Fabrice, P.M. Schmitt, C. Gehin, G. Delhomme, E. McAdams, and A. Dittmar, "Flexible technologies and smart clothing for citizen medicine, home healthcare, and disease prevention," IEEE Transactions on Information Technology in Biomedicine, vol. 9, no. 3, Sep. 2005. 
[11]A. Arcelus, M.H. Jones, R.A. Goubran, and F. Knoefel, "Integration of smart home technologies in a health monitoring system for the elderly," in Proc. $21^{s i}$ International Conference on Advanced Information Networking and Applications Workshops, Niagara Falls, Canada, May 2007, pp. 820-825.

[12] S. Matos, S.S. Birring, I.D. Pavord, and D.H. Evans, "An automated system for 24-h monitoring of cough frequency: The leicester cough monitor," IEEE Transactions on Biomedical Engineering, vol. 54, issue. 8, pp. 1472-1479, Aug. 2007.

[13] S.J. Barry, A.D. Dane, A.H. Morice, and A.D. Walmsley, "The automatic recognition and counting of cough," Cough, vol. 2, Sep. 2006.

[14] M. Barlow, V.V. Varadan, D. Jia, and P. Khoa, "A compact, self-contained cough monitoring system," in Proc. IEEE Region 5 Technical Conference, Fayetteville, USA, Apr. 2007, pp. 167-170.

[15] Y. Hata, S. Kobashi, K. Kuramoto, and H. Nakajima, "Home care system for aging people confined to bed by detached sensor network," in Proc. IEEE Workshop on Robotic Intelligence in Informationally Structured Space (RiiSS), Paris, France, Apr. 201 1, pp. 1-6.

[16]H. Chatrzarrin, A. Arcelus, R. Goubran, and F. Knoefel, "Feature extraction for the differentiation of dry and wet cough sounds," in Proc. IEEE International Symposium on Medical Measurements and Applications, Ban, Italy, May 2011, pp. 162-166.

[17] J. Woodruff, and W. DeLiang, "Directionality-based speech enhancement for hearing aids," in Proc. IEEE International Conference on Acoustic, Speech, and Signal Processing (ICASSP), Prague, Czech Republic, May 2011, pp. 297-300.

[18]B. Cornelis, M. Moonen, and J. Wouters, "Performance analysis of multichannel Wiener filter-based noise reduction in hearing aids under second order statistics estimation errors," IEEE Transactions on Audio, Speech, and Language Processing, vol. 19, issue. 5, pp. 1368-1381, 2011.

[19] M.L. Seltzer, B. Raj, and R.M. Stern, "Speech recognizer-based microphone array processing for robust hands-free speech recognition," in Proc. IEEE Intermational Conference on Acoustics, Speech, and Signal Processing (ICASSP), Orlando, USA, vol. 1, May 2002, pp. 1-897-1-900.

[20] X. Zhao, and Z. Ou, "Closely coupled array processing and model-based compensation for microphone array speech recognition," IEEE Transactions on Audio, Speech, and Language Processing, vol. 15, issue. 3 , pp. 1114-1122, 2007.

[21] H. Wang, and L.Y. Wang, "Multi-sensor adaptive heart and lung sound extraction," in Proc. IEEE Sensors, Toronto, Canada, Oct. 2003, pp. 1096-1099.

[22]L. Qiguang, J. Ea-Ee, and J. Flanagan, "Microphone arrays and speaker identification," IEEE Transactions on Speech and Audio Processing, vol. 2, issue. 4, pp. 622-629, Sep. 1994.

[23] S.M. Griebel, and M.S. Brandstein, "Microphone array source localization using realizable delay vectors," in Proc. IEEE Workshop on the Applications of Signal Processing to Audio and Acoustics, New Paltz, USA, Oct. 2001, pp. 71-74.

[24] D.H. Johnson, and D.E. Dudgeon, Array Signal Processing: Concepts and Techniques, $1^{\text {st }}$ ed., New Jersey: Prentice Hall, 1993.

[25]B.D. Van Veen, and K.M. Buckley, "Beamforming: a versatile approach to spatial filtering," IEEE Acoustic, Speech, Signal Processing Magazine, vol. 5, no. 2, pp. 4-24, Apr. 1988. 
[26] Y.R. Zheng, R.A. Goubran, M. El-Tanany, and H. Shi, "A microphone array system for multimedia applications with near-field signal targets," IEEE Sensors Journal, vol. 5, issue. 6, pp. 1395-1406, Dec. 2005.

[27] Y.R. Zheng, R.A. Goubran, and M. El-Tanany, "A nested sensor array focusing on near field targets," in Proc. IEEE International Conference Sensors, Toronto, Canada, Oct. 2003, pp. 843-848.

[28]A. Gore, A. Fazel, and Sh. Chakrabartty, "Far-field acoustic source localization and bearing estimation using $\sum \Delta$ learners," IEEE Transactions on Circuits and Systems, vol. 57, issue. 4, pp. 783-792, Apr. 2010.

[29] J. Liang, and D. Liu, "Passive localization of mixed near-field and far-field sources using two-stage MUSIC algorithm," IEEE Transactions on Signal Processing, vol. 58, issue. 1, pp. 108-120, Jan. 2010.

[30] A. McKee, and R.A. Goubran, "Beam shape, focus index, and localization error for performance evaluation of a multisensor stethoscope beamformer," in Proc. $26^{\text {th }}$ Annual International Conference of the IEEE EMBS, San Francisco, USA, Sep. 2004, vol. 1, pp. 2062-2065.

[31] M. Kompis, H. Pasterkamp, and G.R. Wodicka, "Acoustic imaging of the human chest," Chest Journal, vol. 120, pp. 1309-\{321, Oct. 2001.

[32] A.R. Abu-El-Quran, R.A. Goubran, and A. Chan, "Security monitoring using microphone arrays and audio classification," IEEE Transactions on Instrumentation and Measurement, vol. 55, issue. 4, Aug. 2004.

[33] H. Saruwatari, H. Kawanami, S. Takeuchi, Y. Takahashi, T. Cincarek, and K. Shikano, "Hands-free speech recognition challenge for real-world speech dialogue systems," in Proc. IEEE International Conference on Acoustics, Speech, and Signal Processing, Taipei, Taiwan, Apr. 2009, pp. 3729-3732.

[34] T. Butko, and C. Nadeu, "On enhancing acoustic event detection by using feature selection and audiovisual feature-level fusion," in Proc. Workshop on Database and Expert Systems Application $(D E X A)$, Bilbao, Spain, Aug. 2010, pp. 271-275.

[35] J. Even, P. Heracleous, C. Ishi, and N. Hagita, "Multi-modal front-end for speaker activity detection in small meetings," in Proc. International Conference on Intelligent Robots and Systems (IROS), San Francisco, USA, Sep. 2011, pp. 536-541.

[36] E. Martinson, T. Apker, and M. Bugajska, "Optimizing a reconfigurable robotic microphone array," in Proc. Intermational Conference on Intelligent Robots and Systems (IROS), San Francisco, USA, Sep. 2011, pp. 125-130.

[37]M.R.P. Thomas, N.D. Gaubitch, J. Gudnason, and P.A. Naylor, "A practical multichannel dereverberation algorithm using multichannel DYPSA and spatiotemporal averaging," in Proc. IEEE Workshop on Applications of Signal Processing to Audio and Acoustics, New Paltz, Oct. 2007, pp. 5053.

[38] H.K. Maganti, D. Gatica-Perez, and I. McCowan, "Speech enhancement and recognition in meetings with an audio-visual sensor array," IEEE Transactions on Audio, Speech, and Language Processing, vol. 15, issue. 8, pp. 2257-2269, 2007.

[39] W. Ser, T.T. Zhang, J. Yu, and J. Zhang, "Detection of wheezes using a wearable distributed array of microphones," in Proc. $6^{\text {th }}$ International Workshop on Wearable and Implantable Body Sensor Networks, Berkley, USA, Jun. 2009, pp. 296-300. 
[40] Y. Hata, S. Kobashi, H. Yamaguchi, O. Ishikawa, N. Tsuchiya, and H. Nakajima, "Human health monitoring system of systems by non-contacted sensors," in Proc. IEEE International Conference on System of Systems Engineering, May 2009, pp. 1-5.

[41] S. Matos, S.S. Birring, I.D. Pavord, and D.H. Evans, "Detection of cough signals in continuous audio recording using Hidden Markov Models," IEEE Transactions on Biomedical Engineering, vol. 53, issue. 6, pp. 1078-1083, Jun. 2006.

[42] S.H. Shin, T. Hashimoto, and S. Hatano, "Automatic detection system for cough sounds as a symptom of abnormal health conditions," IEEE Transactions on Information Technology in Biomedicine, vol. 13 , issue. 4, pp. 486-493, Jul. 2009.

[43] G.A. Fontana, "Before we get starated: what is a cough?," /ung, supp. 1, s3-6, Oct. 2008.

[44] K.F. Chung, D. Bolser, P. Davenport, G. Fontana, A. Morice, and J. Widdicombe, "Semantic and types of cough," Pulmonary Pharmacology and Therapeutics, vol. 22, issue. 2, pp. 139-142, Apr. 2009.

[45] J. Korpas, J. Sadlonova, and M. Vrabec, "Analysis of the cough sound: an overview," Pulmonary Pharmacology, vol. 9, pp. 261-268, Oct. 1996.

[46] A. Murata, Y. Taniguchi, Y. Hashimoto, Y. Kaneko, Y. Takasaki, and S. Kudoh, "Discrimination of productive and non-productive cough by sound analysis," International Medicine, vol. 37, no. 9, pp. 732-735, Sep. 1998.

[47] W. Thorpe, M. Kurver, G. King, and C. Salome, "Acoustic analysis of cough," in Proc. The Seventh Australian and New Zealand Intelligent Information Systems Conference, Perth, Westem Australia, Nov. 2011, pp. 391-394.

[48] R.O. Duda, P.E. Hart, and D.G. Stork, Pattern Classification, $2^{\text {nd }}$ ed., New York: Wiley, 2001.

[49] Sh. Abe, Support Vector Machines for Pattern Classification, London: Springer, 2005.

[50] M. Bal, W. Shen, Q. Hao, and H. Xue, "Collaborative smart home technologies for senior independent living: a review," in Proc. $15^{\text {th }}$ International Conference on Computer Supported Cooperative Work in Design, Lausanne, Switzerland, Jun. 2011, pp. 481-488.

[51] P. Rashidi, and D.J. Cook, "Keeping the resident in the loop: adapting the smart home to the user," IEEE Transaction on Sysems, Man and Cybernetics, Part A: Systems and Humans, vol. 39, no. 5, pp. 949-959, Sep. 2009.

[52]A. Arcelus, R. Goubran, H. Sveistrup, M. Bilodeau, and F. Knoefel, "Context-aware smart home monitoring through pressure measurement sequences," in Proc. IEEE International Workshop on Medical Measurements and Applications MeMeA, Ottawa, Canada, May 2010, pp. 32-37.

[53]R. Nesslerath, J. Haupert, J. Frey, and B. Brandherm, "Supporting persons with special needs in their daily life in a smart home," in Proc. $7^{\text {th }}$ International Conference on Intelligent Emironments, Nottingham, United Kingdom, Jul. 2011, pp. 370-373.

[54] T. Hadidi, and N. Noury, "Correlation between real and simulated data of the activity of the elderly person living independently in a health smart home," in Proc. $13^{\text {th }}$ IEEE International Conference on e-Health Networking Applications and Services (Healthcom), Columbia, USA, Jun. 201 1, pp. 64-69. 
[55] M. Ziefle, C. Rocker, and A. Holzinger, "Medical technology in smart homes: exploring the user's perspective on privacy, intimacy and trust," in Proc. IEEE $35^{\text {th }}$ Annual Computer Software and Applications Conference Workshop (COMPSACW), Munich, Germany, Jul. 2011, pp. 410-415.

[56]B. Mrazovac, M.Z. Bjelica, I. Papp, and N. Teslic, "Smart audio/video playback control based on presence detection and user localization in home environment" in Proc. $2^{\text {nd }}$ Eastern European Regional Conference on the Engineering of Computer Based Systems (ECBS-EERC), Bratislava, Slovakia, Sep. 2011, pp. 44-53.

[57] M. Popescu, and A. Mahnot, "Acoustic fall detection using one-class classifier," in Proc. $31^{\text {st }}$ Annual International Conference of the IEEE EMBS, Minneapolis, USA, Sep. 2009, pp. 3505-3508.

[58] T. Guettari, P.A.C. Aguilar, J. Boudy, H. Medjahed, D. Istrate, J.L. Baldinger, I. Belfeki, M. Opitz, and $M$. Maly-Persy, "Multimodal localization in the context of a medical telemonitoring system," in Proc. $32^{\text {nd }}$ Annual International Conference of the IEEE EMBS, Buenos Aires, Argentina, Aug. 2010, pp. 3835-3838.

[59] Sh. Du, and Sh. Chen, "Weighted support vector machine for classification" in Proc. IEEE Infernational Conference on Syslems, Man and Cybernetics, Hawaii, USA, Oct. 2005, vol. 4 pp. 38663871 .

[60] K. Muller, S. Mika, G. Ratsch, K. Tsuda, and B. Scholkopf, "An introduction to kernel-based learning algorithms," IEEE Transactions on Neural Network, vol. 12, issue. 2, pp. 181-201, 2001.

[61]L. Bo, L. Wang, and L. Jiao, "Training hard-margin support vector machines using greedy stagewise algorithm," IEEE Transactions on Neural Networks, vol. 19, issue. 8, pp. 1446-1455, 2008.

[62] Matlab Bioinformatic Toolbox, http://www.mathworks.com/products/bioinfo/.

[63]C.M. Schmid, R. Feger, S. Scheiblhofer, and A. Stelzer, "Measurement-based delay-and-sum signal processing for linear antenna arrays," in Proc. IEEE International Conference on Wireless Information Technology and Systems (ICWTTS), Hawaii, USA, Aug. 2010, pp. 1-4.

[64]A. Wang, K. Yao, R.E. Hudson, D. Korompis, F. Lorenzelli, S.F. Soli, and S. Gao, "A high performance microphone array system for hearing aid applications," IEEE International Conference on Acoustics, Speech, and Signal Processing, vol. 6, pp. 3197-3200, 1996.

[65] J.M. Kates, "An evaluation of hearing-aid array processing," IEEE ASSP Workshop on Applications of Signal Processing to Audio and Acoustics, New Parltz, USA, Oct. 1995, pp. 15-18. 